\title{
Tuning the Acceptors in Catalyzed Cyclizations Initaited by Allenes. Silylstannylation/Cyclization of Allene-Aldehydes for Synthesis of Polyalkylated Indolizidines including 223A Congeners
}

\author{
Ramaiah Kumareswaran, T. V. RajanBabu* \\ Department of Chemistry, The Ohio State University, 100 West $18^{\text {th }}$ Av., Columbus, OH 43210 \\ SUPPORTING INFORMATION
}

Table of Contents

Regioselective epoxidation of $\mathbf{8}$

S-2

Isomerization of epoxide $\mathbf{1 0}$ to $\mathbf{1 2}$

S-2

Dihydroxylation of $\mathbf{8}$ to $\mathbf{1 3}$

S-3

Oxidation of 21 to 27

$\mathrm{S}-3$

Desilylation of $\mathbf{2 3}$ to $\mathbf{3 8}$

S-4

Dehydration of $\mathbf{3 9}$ to $\mathbf{4 0}$

S-4

Hydrogenation of $\mathbf{4 0}$ to $\mathbf{4 1}$

S-5

Dehydration of $\mathbf{3 8}$ to $\mathbf{4 2}$ and Ir-catalyzed reduction of $\mathbf{4 2}$ to $\mathbf{4 0}$

S-5

${ }^{1} \mathrm{H}$ and ${ }^{13} \mathrm{C}$ NMR spectra of $\mathbf{1 0}$

S-6

${ }^{1} \mathrm{H}$ and ${ }^{13} \mathrm{C}$ NMR spectra of $\mathbf{1 2}$

S-8

${ }^{1} \mathrm{H}$ and ${ }^{13} \mathrm{C}$ NMR spectra of $\mathbf{1 3}$

S-10

${ }^{1} \mathrm{H}$ and ${ }^{13} \mathrm{C}$ NMR spectra of $\mathbf{2 0}$

S-12

${ }^{1} \mathrm{H}$ and ${ }^{13} \mathrm{C}$ NMR spectra of $\mathbf{2 1}$

S-14

${ }^{1} \mathrm{H}$ and ${ }^{13} \mathrm{C}$ NMR spectra of 27

S-16

${ }^{1} \mathrm{H}$ and ${ }^{13} \mathrm{C}$ NMR spectra of 28

S-18

${ }^{1} \mathrm{H}$ and ${ }^{13} \mathrm{C}$ NMR spectra of $\mathbf{2 9}$

S-20

${ }^{1} \mathrm{H}$ and ${ }^{13} \mathrm{C}$ NMR spectra of $\mathbf{3 0}$

$\mathrm{S}-22$

${ }^{1} \mathrm{H}$ and ${ }^{13} \mathrm{C}$ NMR spectra of $\mathbf{3 1}$

S-24

${ }^{1} \mathrm{H}$ and ${ }^{13} \mathrm{C}$ NMR spectra of $\mathbf{3 2}$

S-26

Gas chromatogram of $\mathbf{3 2}$

S-29

${ }^{1} \mathrm{H}$ and ${ }^{13} \mathrm{C}$ NMR spectra of $\mathbf{2 2}$

S-30

${ }^{1} \mathrm{H}$ and ${ }^{13} \mathrm{C}$ NMR spectra of $\mathbf{3 3}$

S-32

${ }^{1} \mathrm{H}$ and ${ }^{13} \mathrm{C}$ NMR spectra of $\mathbf{3 5}$

$\mathrm{S}-34$

${ }^{1} \mathrm{H}$ and ${ }^{13} \mathrm{C}$ NMR spectra of $\mathbf{3 6}$

S-36

${ }^{1} \mathrm{H}$ and ${ }^{13} \mathrm{C}$ NMR spectra of $\mathbf{3 7}$

S-38

Gas chromatogram of $\mathbf{3 7}$

S-40

${ }^{1} \mathrm{H}$ and ${ }^{13} \mathrm{C}$ NMR spectra of $\mathbf{2 3}$

S-41

${ }^{1} \mathrm{H}$ and ${ }^{13} \mathrm{C}$ NMR spectra of $\mathbf{3 8}$

S-43

${ }^{1} \mathrm{H}$ and ${ }^{13} \mathrm{C}$ NMR spectra of $\mathbf{3 9}$

S-45

${ }^{1} \mathrm{H}$ and ${ }^{13} \mathrm{C}$ NMR spectra of $\mathbf{4 0}$

S-47

${ }^{1} \mathrm{H}$ and ${ }^{13} \mathrm{C}$ NMR spectra of $\mathbf{4 1}$

S-49

${ }^{1} \mathrm{H}$ and ${ }^{13} \mathrm{C}$ NMR spectra of $\mathbf{2 4}$

S-51

ORTEP representations of $\mathbf{2 2 ,} 28$ and $\mathbf{3 8}$

S-53 
Regioselective epoxidation of 8 (and 5-epi-8) to 6-(2-(tert-butyldimethylsilyl)oxiran-2-yl)hexahydro-7-methylene-5-propylindolizin-3(5H)-one (10). To a stirred solution of 8 (and 5epi-8) and $(0.5 \mathrm{mmol}, 167 \mathrm{mg})$ in $5 \mathrm{~mL}$ of $\mathrm{CH}_{2} \mathrm{Cl}_{2}$ at $0^{\circ} \mathrm{C}$ was added $70 \% \mathrm{~m}$-CPBA $(0.6 \mathrm{mmol}$, $147 \mathrm{mg}$ ) in one portion and the resultant mixture was brought to room temperature, and stirred for $24 \mathrm{~h}$. The reaction mixture was diluted with $50 \mathrm{~mL}$ of $\mathrm{CH}_{2} \mathrm{Cl}_{2}$ and transferred into a separatory funnel. Successive washings with aqueous sat. $\mathrm{NaHCO}_{3}(2 \times 30 \mathrm{~mL})$ and brine, drying $\left(\mathrm{MgSO}_{4}\right)$ and concentration afforded a crude material which was purified by flash column chromatography (15\% EtOAc in hexanes) to get $\mathbf{1 0}$ as an inseparable diastereomeric mixture (93 mg, 53\%): Major epoxide 10: ${ }^{1} \mathrm{H}$ NMR (500 MHz) $\square 5.69(\mathrm{~d}, \mathrm{~J}=3.5 \mathrm{~Hz}, 1 \mathrm{H}), 5.6(\mathrm{~d}, \mathrm{~J}=3.5 \mathrm{~Hz}$, $1 \mathrm{H}), 4.45(\mathrm{~d}, \mathrm{~J}=9 \mathrm{~Hz}, 1 \mathrm{H}), 4.13(\mathrm{~d}, \mathrm{~J}=11.5 \mathrm{~Hz}, 1 \mathrm{H}), 3.95(\mathrm{~d}, \mathrm{~J}=11.5 \mathrm{~Hz}, 1 \mathrm{H}), 3.79-3.72(\mathrm{~m}$, 1H), 2.44-2.4 (m, 3H), 2.3-2.21 (m, 1H), $2.07(\mathrm{dd}, \mathrm{J}=17,11 \mathrm{~Hz}, 1 \mathrm{H}), 1.78-1.66(\mathrm{~m}, 2 \mathrm{H}), 1.37-$ $1.16(\mathrm{~m}, 4 \mathrm{H}), 0.93(\mathrm{~s}, 9 \mathrm{H}), 0.88(\mathrm{t}, \mathrm{J}=7 \mathrm{~Hz}, 3 \mathrm{H}), 0.12(\mathrm{~s}, 3 \mathrm{H}), 0.04(\mathrm{~s}, 3 \mathrm{H}) ;{ }^{13} \mathrm{C}$ NMR $(125 \mathrm{MHz})$ $\square 173.4,142.8,132.8,57.1,51.9,50.8,48.3,44.6,42.9,30.6,28.6,27.4,24.5,19.8,17.8,14.2$, 5.8 .

Isomerization of epoxide 10 to (E)-6-(1-(tert-butyldimethylsilyl)-2-hydroxyethyli- dene) hexahydro-7-methylene-5-propylindolizin-3(5H)-one (12). To a stirred solution of the epoxide $10(0.23 \mathrm{mmol}, 80 \mathrm{mg})$ in acetone or $\mathrm{MeOH}(4 \mathrm{~mL})$ was added $1 \mathrm{~mL}$ of $10 \mathrm{~N}$ aq. $\mathrm{H}_{2} \mathrm{SO}_{4}$ and the mixture was refluxed for $2 \mathrm{~h}$. Analysis by TLC indicated the formation of a polar spot with a disappearance of the starting material. The reaction mixture was concentrated on a rotary evaporator. The residue was dissolved in $50 \mathrm{~mL}$ of $\mathrm{CH}_{2} \mathrm{Cl}_{2}$, transferred into a separatory funnel and washed successively with aq. $\mathrm{NaHCO}_{3}$ and brine, and dried $\left(\mathrm{MgSO}_{4}\right)$. Evaporation of the solvent gave a crude product which was purified by flash column chromatography (35\% EtOAc in hexanes) to get $12(24 \mathrm{mg}, 30 \%)$ as a viscous oil: ${ }^{1} \mathrm{H}$ NMR $(500 \mathrm{MHz}) \square 5.68(\mathrm{~d}, \mathrm{~J}=3.5 \mathrm{~Hz}$, $1 \mathrm{H}), 5.59(\mathrm{~d}, \mathrm{~J}=3.5 \mathrm{~Hz}, 1 \mathrm{H}), 4.45(\mathrm{~d}, \mathrm{~J}=9 \mathrm{~Hz}, 1 \mathrm{H}), 4.13(\mathrm{dd}, \mathrm{J}=11.5,4.5 \mathrm{~Hz}, 1 \mathrm{H}), 3.95(\mathrm{dd}, \mathrm{J}$ $=11.5,6 \mathrm{~Hz}, 1 \mathrm{H}), 3.79-3.74(\mathrm{~m}, 1 \mathrm{H}), 2.45(\mathrm{dd}, \mathrm{J}=11,5 \mathrm{~Hz}, 1 \mathrm{H}), 2.42(\mathrm{t}, \mathrm{J}=7 \mathrm{~Hz}, 2 \mathrm{H}), 2.29-$ $2.23(\mathrm{~m}, 1 \mathrm{H}), 2.07(\mathrm{dd}, \mathrm{J}=17,11 \mathrm{~Hz}, 1 \mathrm{H}), 1.78-1.7(\mathrm{~m}, 2 \mathrm{H}), 1.33-1.26(\mathrm{M}, 3 \mathrm{H}), 1.18(\mathrm{t}, \mathrm{J}=5.5$ $\mathrm{Hz}, 1 \mathrm{H}$ exchangeable with $\left.\mathrm{D}_{2} \mathrm{O}\right), 0.93(\mathrm{~s}, 9 \mathrm{H}), 0.86(\mathrm{t}, \mathrm{J}=7 \mathrm{~Hz}, 3 \mathrm{H}), 0.15(\mathrm{~s}, 3 \mathrm{H}), 0.07$ (s, 3H); ${ }^{13} \mathrm{C}$ NMR (125 MHz) $\square$ 173.4, 150.2, 143.3, 131.5, 126.9, 64, 50.7, 50.2, 34.3, 33.6, 30.1, 27.3, 24.7, 19.6, 17.8, 14.4, -3.4, -5; HRMS (EI/CI) calcd for $\mathrm{C}_{20} \mathrm{H}_{35} \mathrm{NO}_{2} \mathrm{SiNa}^{+} 372.232924$, found 372.231673 . 


\section{Dihydroxylation of 8 (and 5-epi-8) to 6-(1-(tert-butyldimethylsilyl)-1,2-dihydroxy-}

ethyl)hexahydro-7-methylene-5-propylindolizin-3(5H)-one (13). In a single-necked flask equipped with a stirring bar were placed 8 (and 5-epi-8) $(0.5 \mathrm{mmol}, 167 \mathrm{mg})$ in $2 \mathrm{~mL}$ of acetone and $1 \mathrm{~mL}$ of water at $\mathrm{rt}$. Osmium tetroxide $(50 \mu \mathrm{L}$ from a $3.7 \% \mathrm{w} / \mathrm{w}$ solution in $\mathrm{t}-\mathrm{BuOH})$ and NMO (1 mmol, $117 \mathrm{mg}$ ) were added successively, and the resulting dark mixture was stirred for two days. Analysis by TLC indicated the formation of a polar spot corresponding to the expected diol along with starting material which did not decrease with time. The reaction mixture was diluted with $\mathrm{CH}_{2} \mathrm{Cl}_{2}(50 \mathrm{~mL})$ and filtered through a pad of $\mathrm{MgSO}_{4}$. The filtrate was transferred into a separatory funnel, washed with water and brine, dried $\left(\mathrm{MgSO}_{4}\right)$ and concentrated to get a crude material. Purification by flash column chromatography (1:1 EtOAc and hexanes) afforded pure 13 (42 $\mathrm{mg}, 23 \%)$ as colorless solid: $\mathrm{mp} 112-114^{\circ} \mathrm{C} ;{ }^{1} \mathrm{H}$ NMR (500 $\mathrm{MHz}) \square 5.9(\mathrm{~d}, \mathrm{~J}=1.5 \mathrm{~Hz}, 1 \mathrm{H}), 5.87$ (d, J = 1.5 Hz, 1H), 4.3-4.26 (m, 1H), 4.04 (d, J = $11 \mathrm{~Hz}$, 1H), 3.89 (dd, J = 11, $9 \mathrm{~Hz}, 1 \mathrm{H}), 3.76-3.7(\mathrm{~m}, 1 \mathrm{H}), 2.89$ (d, J = 5.5 Hz, 1H), 2.47 (dd, J = 12.5 Hz, 1H), 2.45-2.41 (m, 2H), 2.25-2.22 (m, 1H), 2.14 (dd, J = 9, 2 Hz, 1H), 1.7-1.65 (m, 2H), 1.3$1.22(\mathrm{~m}, 3 \mathrm{H}), 1.19(\mathrm{td}, \mathrm{J}=12.5,1.5 \mathrm{~Hz}, 1 \mathrm{H}), 0.93$ (s. 9H), 0.87 (t, J = $7 \mathrm{~Hz}, 3 \mathrm{H}), 0.15(\mathrm{~s}, 3 \mathrm{H})$, $0.11(\mathrm{~s}, 3 \mathrm{H}) ;{ }^{13} \mathrm{C}$ NMR (125 MHz) $\square 173.7,147.3,133.3,75.9,64.9,53.4,51.4,50.9,43,31.1$, 30.2, 27.4, 24.1, 20.9, 17.6, 14.3, -4.9, -5; HRMS (EI/CI) calcd for $\mathrm{C}_{20} \mathrm{H}_{37} \mathrm{NO}_{3} \mathrm{SiNa}^{+} 390.243489$, found 390.24543 .

\section{Oxidation of 21 to 8-ethyl-5-propyl-6-(1-trimethylsilanyl-vinyl)-hexahydro-indolizine-3, 7-} dione (27). In a single-necked flask equipped with a stirring bar and a $\mathrm{N}_{2}$ inlet were placed PCC (0.65 mmol, $140 \mathrm{mg})$, celite $(150 \mathrm{mg})$ and powdered $4 \square$ molecular sieves (150 mg). The substrate $(0.22 \mathrm{mmol}, 71 \mathrm{mg})$ dissolved in $\mathrm{CH}_{2} \mathrm{Cl}_{2}$ was added and the resultant dark mixture was stirred at $\mathrm{rt}$ for $4 \mathrm{~h}$. Analysis by TLC indicated the disappearance of the starting material while showing a non-polar spot. The reaction mixture was diluted with ether $(20 \mathrm{~mL})$ and filtered through a pad of $\mathrm{MgSO}_{4}$. The filtrate was concentrated to get a crude material which was purified by flash column chromatography (15\% EtOAc in hexanes) to get 27 as an oil (58 $\mathrm{mg}$, $82 \%) .{ }^{1} \mathrm{H}$ NMR $\left(500 \mathrm{MHz}, \mathrm{CDCl}_{3}\right) \square 5.86(\mathrm{~s}, 1 \mathrm{H}), 5.62$ (s, 1H), 4.49-4.45 (m, 1H), 4.24 (q, J = 6 $\mathrm{Hz}, 1 \mathrm{H}), 3.22(\mathrm{~d}, \mathrm{~J}=3.5 \mathrm{~Hz}, 1 \mathrm{H}), 2.54(\mathrm{dt}, \mathrm{J}=10,5 \mathrm{~Hz}, 1 \mathrm{H}), 2.48-2.36(\mathrm{~m}, 2 \mathrm{H}), 2.16-2.1(\mathrm{~m}$, $1 \mathrm{H}), 1.88-1.82(\mathrm{~m}, 1 \mathrm{H}), 1.73-1.59(\mathrm{~m}, 3 \mathrm{H}), 1.52-1.43(\mathrm{~m}, 1 \mathrm{H}), 1.36-1.28(\mathrm{~m}, 2 \mathrm{H}), 0.92(\mathrm{t}, \mathrm{J}=7$ $\mathrm{Hz}, 3 \mathrm{H}), 0.9$ (t, J = $7 \mathrm{~Hz}, 3 \mathrm{H}), 0.1$ (s, 9H); ${ }^{13} \mathrm{C}$ NMR (125 MHz) $\square 209,173.7,149.5,129.3,60.6$, 
56.8, 55.3, 53.2, 36.6, 31.2, 21.5, 19.1, 18.9, 14.1, 12.7, -0.4; HRMS (EI/CI) calcd for $\mathrm{C}_{18} \mathrm{H}_{31} \mathrm{NO}_{2} \mathrm{SiNa}^{+}$344.201624, found 344.20180.

Desilylation of 23 to 8-ethyl-7-hydroxy-5-propyl-6-vinyl-hexahydro-indolizin-3-one (38). Experimental procedure is the same as for the desilylation of $\mathbf{2 1}$ to $\mathbf{2 8}$. Heating of $\mathbf{2 3}(0.74$ mmol, $240 \mathrm{mg}$ ) with TBAF in THF (3 mmol, $3 \mathrm{~mL})$ in THF (4 mL) and DMSO (2 mL) for $3 \mathrm{~h}$ followed by work up and purification by flash column chromatography (15\% EtOAc in hexanes) afforded 38 (120 mg, 65\%) as a solid: mp 106-108 ${ }^{\circ} \mathrm{C} ;{ }^{1} \mathrm{H}$ NMR (500 $\left.\mathrm{MHz}, \mathrm{CDCl}_{3}\right) \square 5.82-5.75$ (m, 1H), $5.21(\mathrm{dd}, \mathrm{J}=10,1.5 \mathrm{~Hz}, 1 \mathrm{H}), 4.16(\mathrm{dt}, \mathrm{J}=17,1.5 \mathrm{~Hz}, 1 \mathrm{H}), 4.07$ (dt, J = 8.5, $4 \mathrm{~Hz}, 1 \mathrm{H})$, $3.9(\mathrm{t}, \mathrm{J}=2.6 \mathrm{~Hz}, 1 \mathrm{H}), 3.46(\mathrm{td}, \mathrm{J}=11.5,3.5 \mathrm{~Hz}, 1 \mathrm{H}), 2.37-2.29(\mathrm{~m}, 3 \mathrm{H}), 2.24-2.27(\mathrm{~m}, 1 \mathrm{H})$, 2.05-1.99 (m, 2H), 1.85-1.78 (m, 1H), 1.76-1.68 (m, 1H), $1.64(\mathrm{dq}, \mathrm{J}=10,4$ Hz, 1H), 1.59-1.48 $(\mathrm{m}, 2 \mathrm{H}), 1.37-1.19(\mathrm{~m}, 2 \mathrm{H}), 0.96(\mathrm{t}, \mathrm{J}=7.5 \mathrm{~Hz}, 3 \mathrm{H}), 0.9(\mathrm{t}, 7.5 \mathrm{~Hz}, 3 \mathrm{H}) ;{ }^{13} \mathrm{C} \mathrm{NMR}(125 \mathrm{MHz})$ $\square 174.7,137.9,118.5,72.3,57.8,55.4,47.1,45.6,33.1,31,20.6,20.1,18,14.4,12.7$.

Hydrogenation of 38 to 6, 8-diethyl-7-hydroxy-5-propyl-hexahydro-indolizin-3-one (39). In a pressure tube $5 \% \mathrm{Pd}-\mathrm{C}$ was placed. $\mathrm{An} \mathrm{EtOH}(3 \mathrm{~mL})$ solution of $38(0.36 \mathrm{mmol}, 91 \mathrm{mg})$ was added and the tube was evacuated and refilled with $\mathrm{H}_{2}$ at 60 psi alternatively three times. The reaction mixture was stirred for $7 \mathrm{~h}$ and then filtered through a pad of $\mathrm{MgSO}_{4}$. The pad was washed with $25 \mathrm{~mL}$ of hot $\mathrm{MeOH}$. The filtrate was concentrated and the residue was subjected to flash column chromatography (25\% EtOAc in hexanes) to get 39 (76 mg, 84\%) as solid: mp 103$105^{\circ} \mathrm{C} ;{ }^{1} \mathrm{H}$ NMR $\left(500 \mathrm{MHz}, \mathrm{CDCl}_{3}\right) \square 4.04(\mathrm{q}, \mathrm{J}=5.6 \mathrm{~Hz}, 1 \mathrm{H}), 4(\mathrm{~d}, 2.5 \mathrm{~Hz}, 1 \mathrm{H}), 3.26(\mathrm{td}, \mathrm{J}=$ 11.5, $4.5 \mathrm{~Hz}, 1 \mathrm{H}), 2.54$ (br s, 1H, exchangeable with $\mathrm{D}_{2} \mathrm{O}$ ), 2.27 (dt, J = 11, $\left.7 \mathrm{~Hz}, 2 \mathrm{H}\right), 2.24-2.1$ (m, 1H), 2.05-1.91 (m, 1H), 1.88-1.78 (m, 1H), 1.72-1.58 (m, 2H), 1.55-1.42 (m, 4H), 1.35-1.19 $(\mathrm{m}, 2 \mathrm{H}), 1.18-1.08(\mathrm{~m}, 1 \mathrm{H}), 0.94-0.86(\mathrm{~m}, 9 \mathrm{H}) ;{ }^{13} \mathrm{C} \mathrm{NMR}(125 \mathrm{MHz}) \square 174.5,67.6,58.1,57$, 47.5, 40.6, 33.2, 30.2, 20.6, 20.3, 20.1, 18.1, 14.5, 12.5, 11.9; HRMS (EI/CI) calcd for $\mathrm{C}_{15} \mathrm{H}_{27} \mathrm{NO}_{2} \mathrm{Na}^{+}$276.193397, found 276.194228.

Dehydration of 39 to 6, 8-diethyl-5-propyl-1,5,8,8a-tetrahydro-2H-indolizin-3-one (40). In a two-necked flask equipped with a stirring bar, rubber septum and a $\mathrm{N}_{2}$ inlet was placed 39 (0.296 mmol, $75 \mathrm{mg}$ ) in $1.5 \mathrm{~mL}$ of dry pyridine at $\mathrm{rt}$. To it was added $\mathrm{POCl}_{3}(1 \mathrm{~mL})$ dropwise. After stirring $5 \mathrm{~h}$, the reaction mixture was concentrated to dryness and loaded directly on a column packed with $\mathrm{SiO}_{2}$. Elusion with 20\% EtOAc in hexanes gave 40 (42 mg, 60\%) as colorless oil: ${ }^{1} \mathrm{H}$ NMR (500 MHz, $\left.\mathrm{CDCl}_{3}\right) \square 5.75$ (d, J = 7 Hz, 1H), 4.03 (br s, 1H), 3.57 (ddd, J = 10.5, 10.5, 4 $\mathrm{Hz}, 1 \mathrm{H}), 2.48-2.36(\mathrm{~m}, 2 \mathrm{H}), 2.31-2.24(\mathrm{~m}, 1 \mathrm{H}), 2.13-2.08(\mathrm{~m}, 1 \mathrm{H}), 2.06(\mathrm{q}, \mathrm{J}=7.5 \mathrm{~Hz}, 1 \mathrm{H}), 1.95$ 
$(\mathrm{qu}, \mathrm{J}=7.5 \mathrm{~Hz}, 1 \mathrm{H}), 1.85-1.78(\mathrm{~m}, 2 \mathrm{H}), 1.61-1.54(\mathrm{~m}, 2 \mathrm{H}), 1.27-1.21(\mathrm{~m}, 2 \mathrm{H}), 1.7-1.1(\mathrm{~m}, 1 \mathrm{H})$, $1.03(\mathrm{t}, \mathrm{J}=7 \mathrm{~Hz}, 3 \mathrm{H}), 0.95(\mathrm{t}, \mathrm{J}=7 \mathrm{~Hz}, 3 \mathrm{H}), 0.86(\mathrm{t}, \mathrm{J}=7 \mathrm{~Hz}, 3 \mathrm{H}) ;{ }^{13} \mathrm{C} \mathrm{NMR}(125 \mathrm{MHz})$ $\square 177.1,140.1,124,59.4,55.7,38.5,33.3,32.4,26.6,22.8,21.9,17.8,14.7,12.9,12.5$.

Hydrogenation of 40 to 6, 8-Diethyl-5-propyl-hexahydro-indolizin-3-one (41). In a pressure tube $40(0.17 \mathrm{mmol}, 40 \mathrm{mg})$ and $30 \mathrm{mg}$ of $5 \% \mathrm{PtO}_{2}-\mathrm{C}(30 \mathrm{mg})$ in $4 \mathrm{~mL}$ of ethanol were placed. The tube was evacuated and refilled with $\mathrm{H}_{2}$ (60 psi) alternatively thrice and stirred overnight. The reaction mixture was exposed to air and filtered through a pad of $\mathrm{MgSO}_{4}$. The pad was washed with $30 \mathrm{~mL}$ of hot $\mathrm{MeOH}$. The filtrate was concentrated and purified by flash column chromatography (20\% EtOAc in hexanes) to get mixture of $\mathbf{4 1}$ and an unidentified component (35 mg, 88\%). 41: ${ }^{1} \mathrm{H} \mathrm{NMR}\left(500 \mathrm{MHz}, \mathrm{CDCl}_{3}\right) \square 3.83$ (dt, J = 8.9, $\left.6 \mathrm{~Hz}, 1 \mathrm{H}\right), 3.59$ (dt, J = 7.6, $5.2 \mathrm{~Hz}, 1 \mathrm{H}), 1.85-1.73$ (m, 7H from both the isomers), 1.55-1.4 (m, 7H), 1.25-1.4 (m, 6H), 0.95$0.85(\mathrm{~m}, 13 \mathrm{H}) ;{ }^{13} \mathrm{C}$ NMR $(125 \mathrm{MHz}) \square 176.07,59.4,55.2,38.6,36.7,32.9,32.2,28.7,24.8,23.1$, 22.2, 21.5, 14.8, 12.7, 12.3, 12 .

Dehydration of 38 to 8-ethyl-5-propyl-6-vinyl-1, 5, 8, 8a-tetrahydro-2H-indolizin-3-one (42) and Ir-catalyzed reduction of 42 to 40 . In a two-necked flask equipped with a rubber septum, $\mathrm{N}_{2}$ inlet and a stirring bar was placed $38(0.2 \mathrm{mmol}, 50 \mathrm{mg})$ in $2 \mathrm{~mL}$ of pyridine at $\mathrm{rt}$. Phosphorus oxychloride was added dropwise and the resultant mixture was stirred for $5 \mathrm{~h}$. Analysis by TLC indicated a complete consumption of the starting material. The reaction mixture was concentrated to dryness, redissolved in $50 \mathrm{~mL}$ of $\mathrm{CH}_{2} \mathrm{Cl}_{2}$, transferred into a separatory funnel and washed with water and brine successively. The organic part was dried $\left(\mathrm{MgSO}_{4}\right)$ and concentrated to get unstable $42(16 \mathrm{mg})$. Treatment of a dichloromethane $(3 \mathrm{~mL})$ solution of 42 with Crabtree catalyst $(0.006 \mathrm{mmol}, 5 \mathrm{mg})$ at $200 \mathrm{psi}_{2}$ pressure for $12 \mathrm{~h}$ led to the reduction of only the exocyclic olefin giving $40(13 \mathrm{mg}, 79 \%)$ as an oil after flash column chromatography. This compound was identified by comparison of spectroscopic data with those of a smple prepared previously (vide supra). 


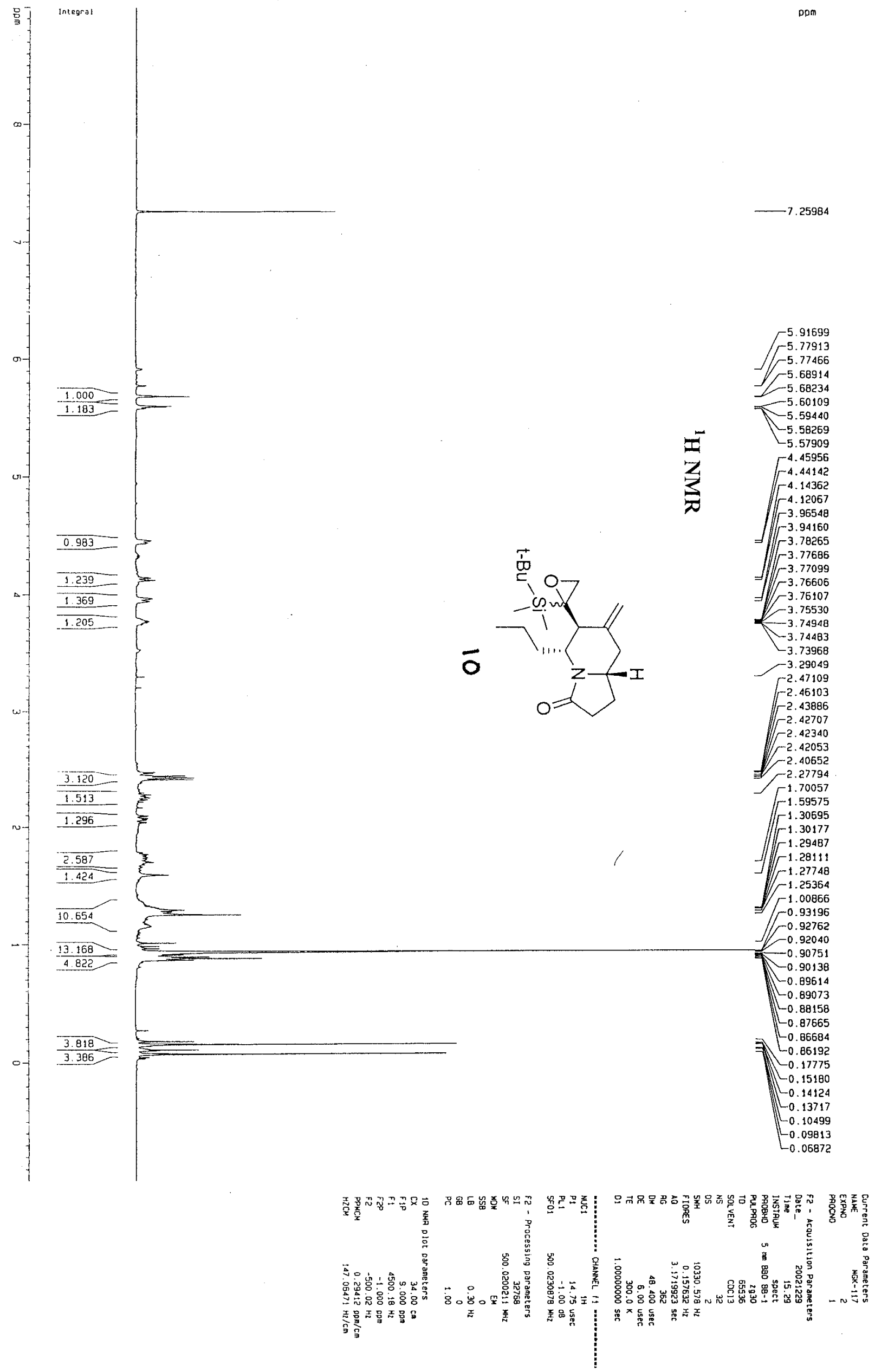




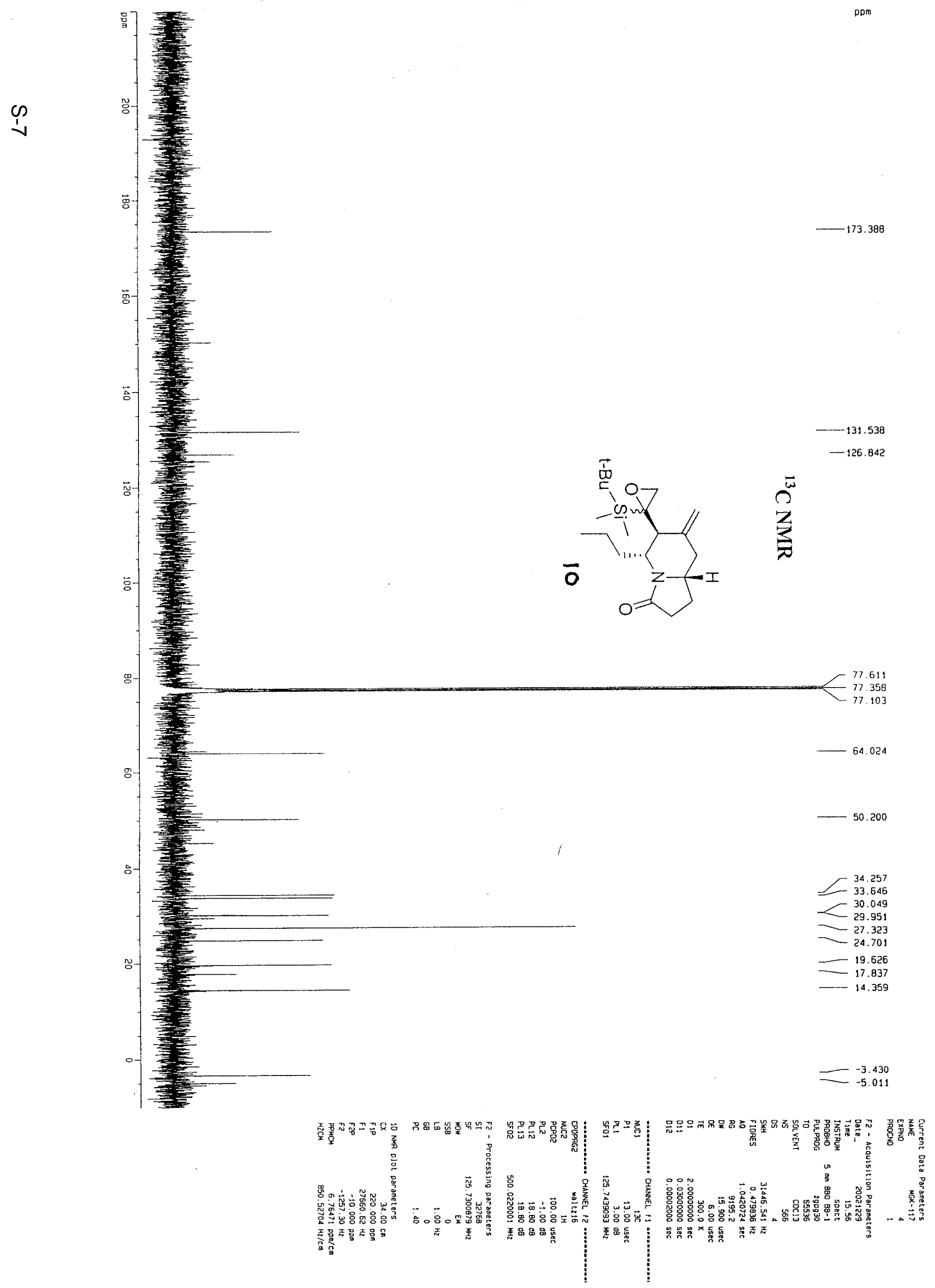




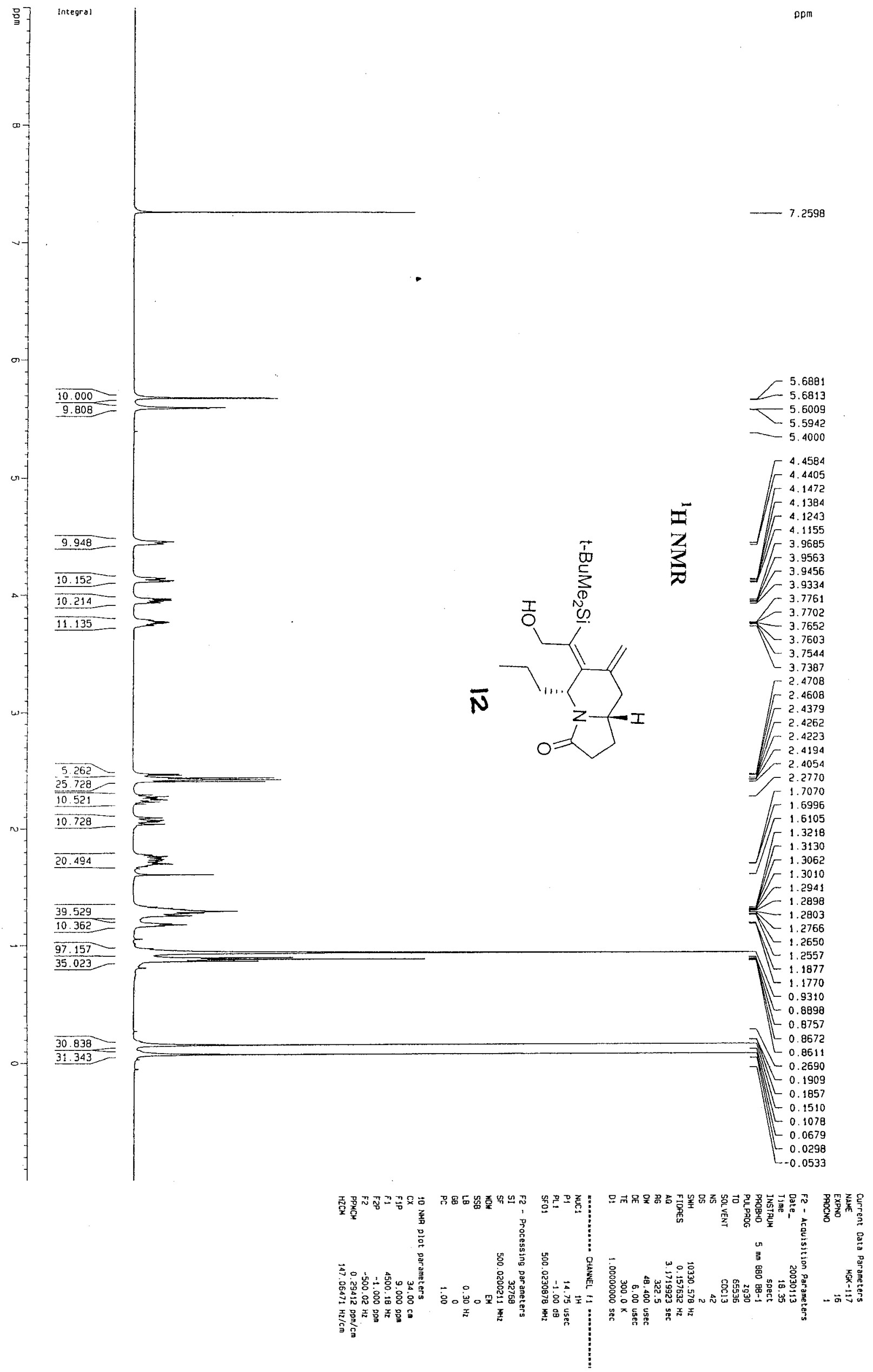


@
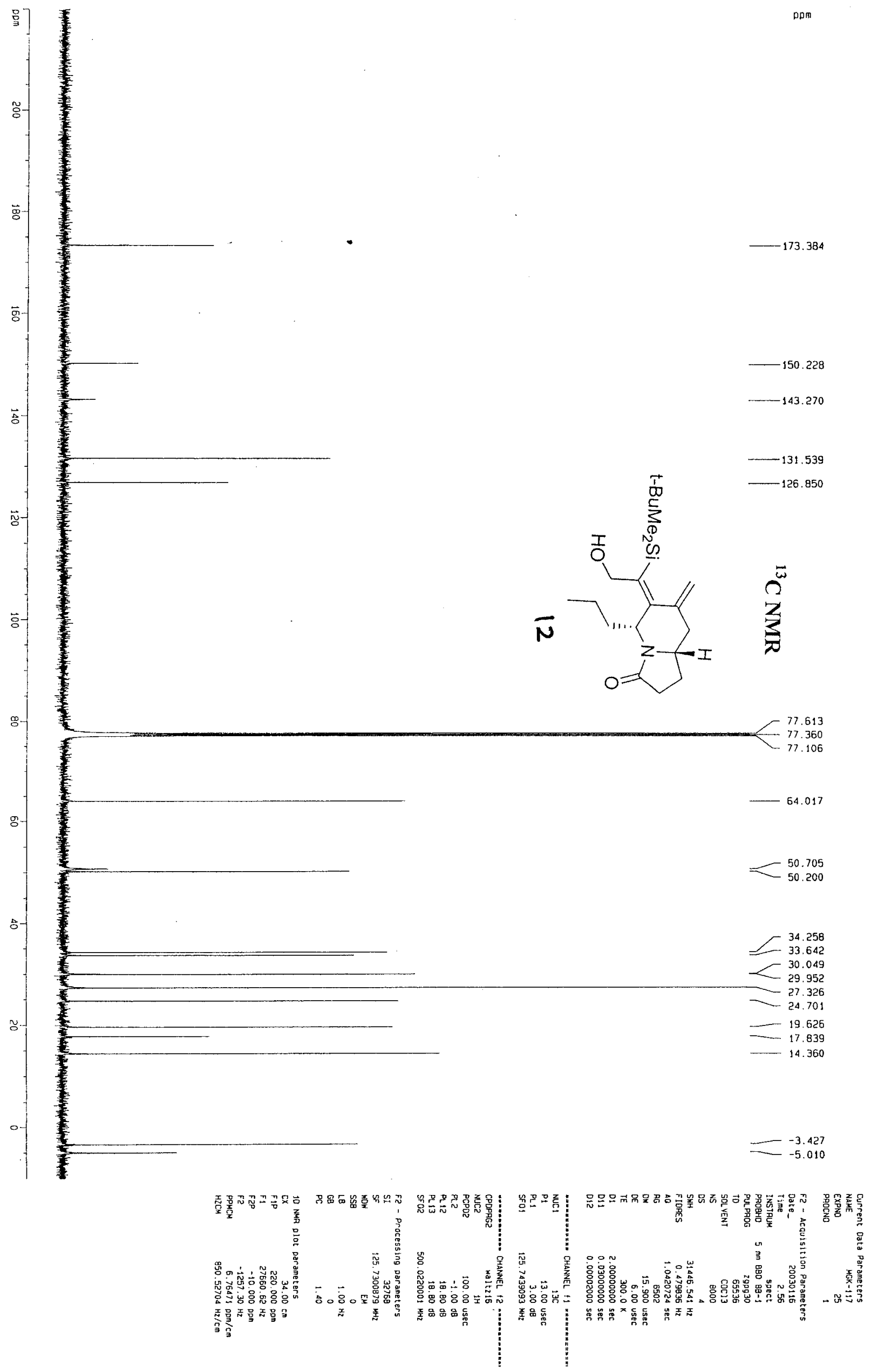


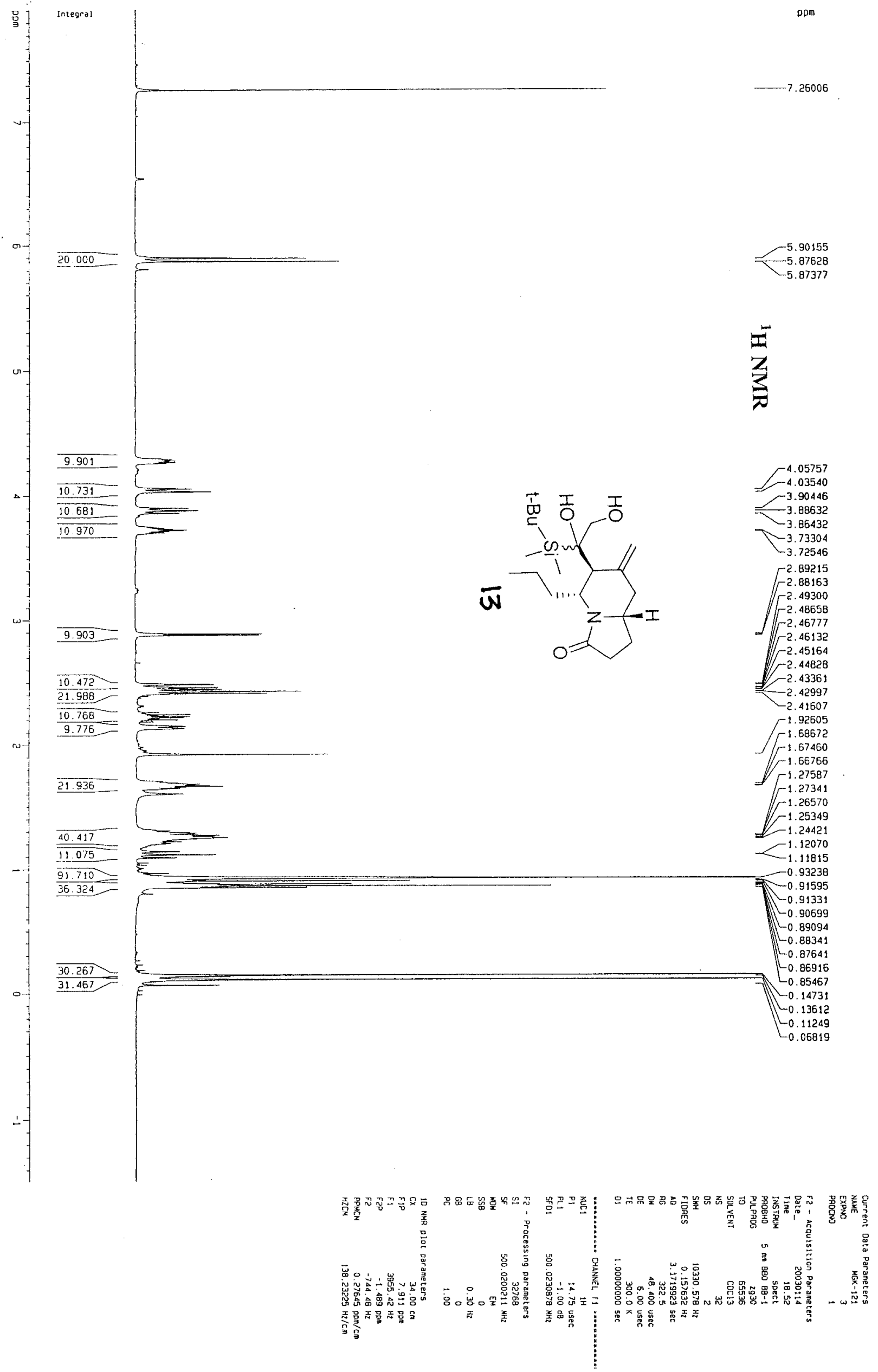




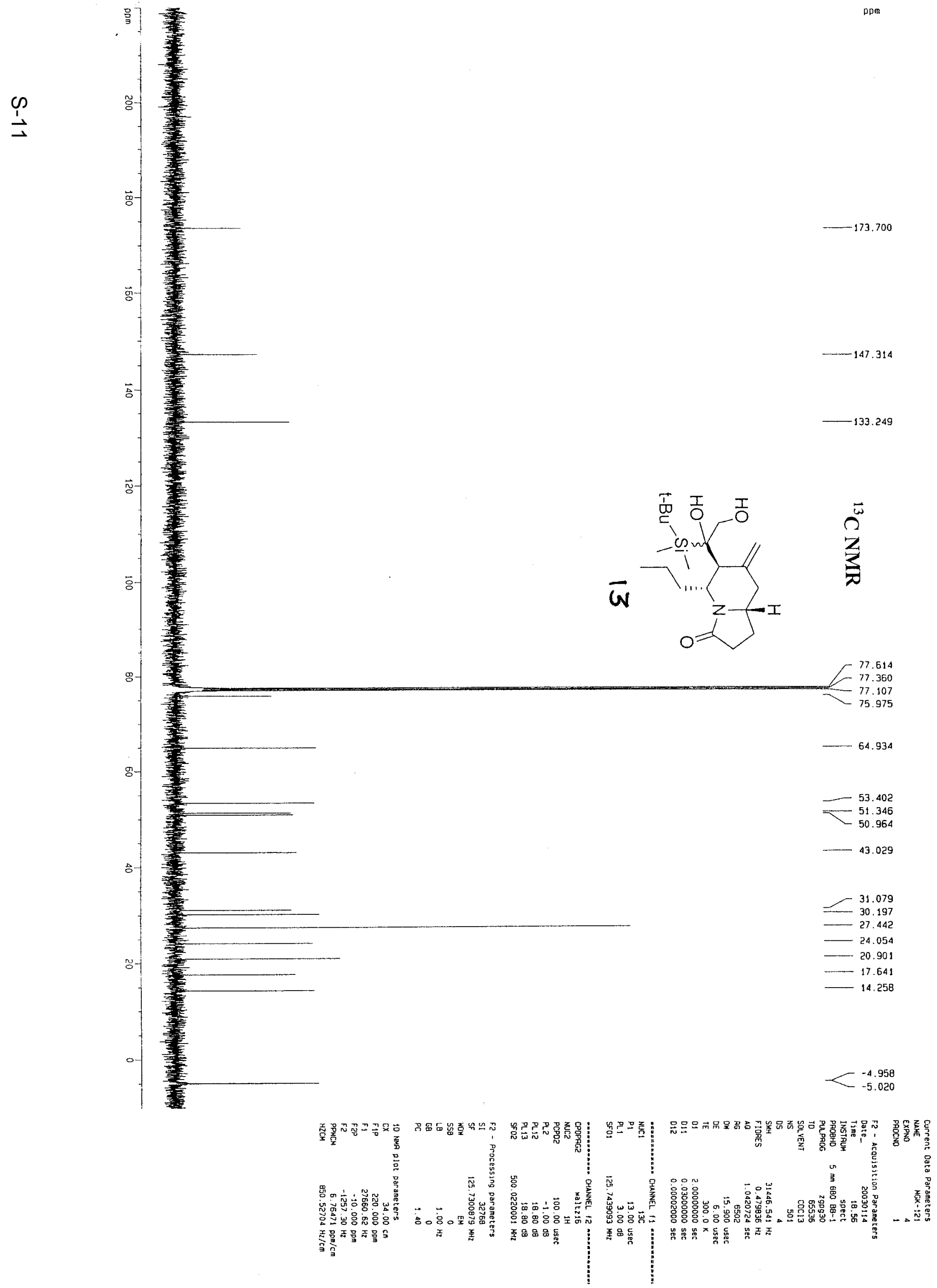



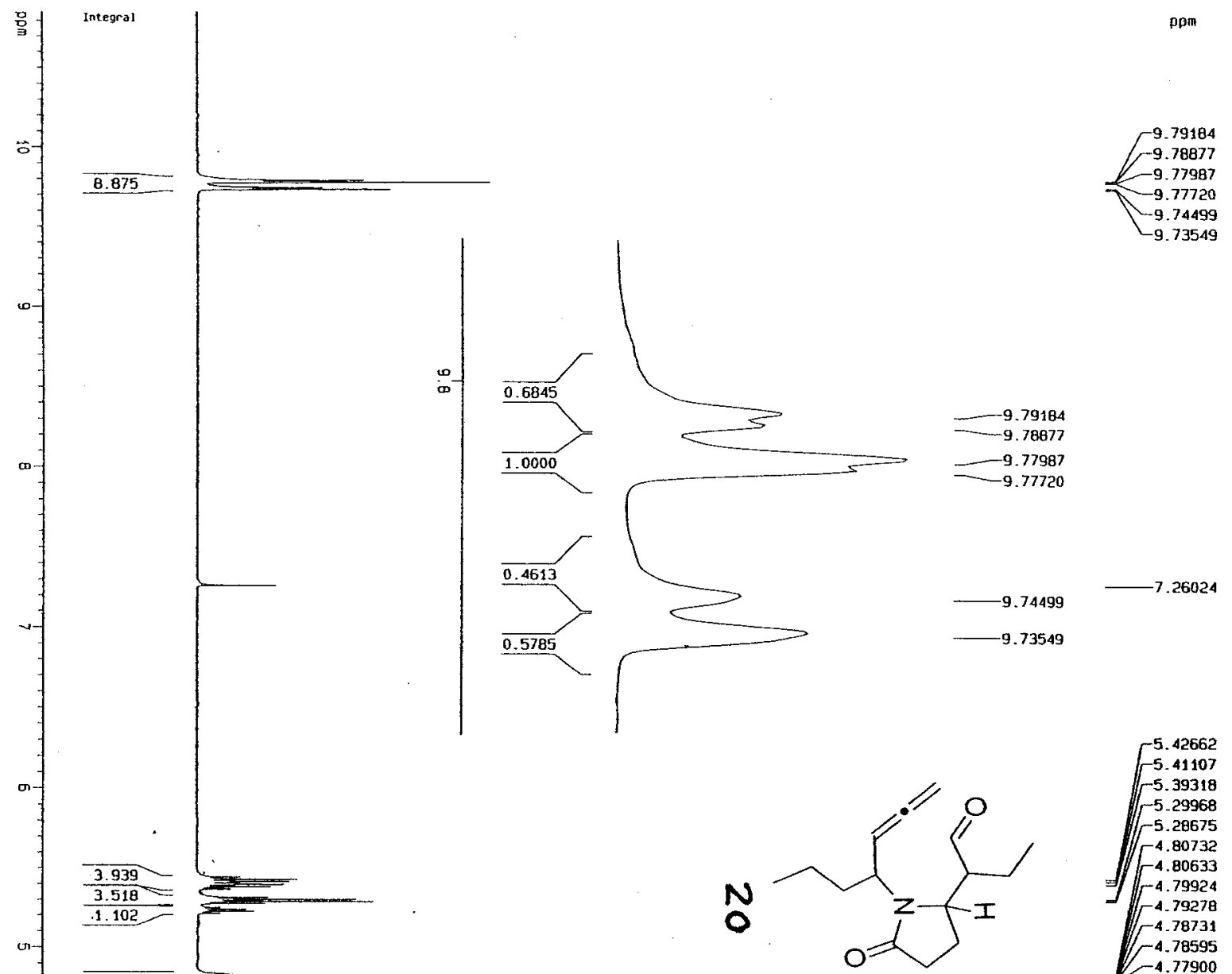

saese $-5.41107$ $-5.39318$ -5.28675 $\Gamma^{-5.28675}$ $\int \Gamma^{4.80732}$ $\frac{-.518}{.1 .102}$

5.072

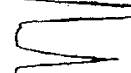

1.835

$\frac{1.635}{3.610}=$

$\frac{2.502}{1.839}$

5

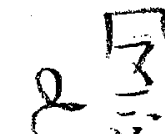

3.817

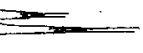

$\mathcal{x}$

a 5

-4.80633
-4.79924

4.79924
-4.79278

4.79279
-4.78731

$r^{4.78595}$

$-4.77900$

$-4.77438$

$-4.77036$

-4.77036
-4.76605
-4.76105

4.75887

$-4.75705$

4.75291

$-4.14686$

-3.82447
-3.80618

क व

$\left\{\begin{array}{l}\frac{3.716}{1.160}=\frac{1.895}{2.339} \\ \frac{20.696}{20.993} \\ \frac{42.369}{4.348} \\ \frac{28.971}{65.126}\end{array}\right.$

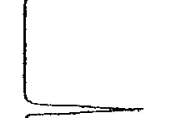

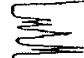
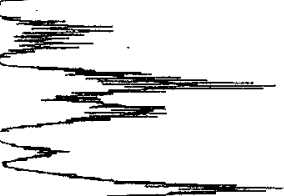

0

30

2.32338

$r^{2.32338}$

$\left[\begin{array}{r}2.30062 \\ -2.30374\end{array}\right.$

$-2.30004$ $\Gamma^{-2.28228}$

$-1.79447$

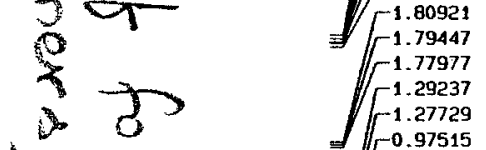

$1 \mathrm{C}=\Gamma_{-0.97515}^{0.96530}$

0.96013

$-0.95633$

0.95053 0.94168
-0.93565

0.93565
-0.90760

$-0.69882$

$-0.88971$

$[0.88438$

$L_{0.87814}^{0.88438}$

0.86971

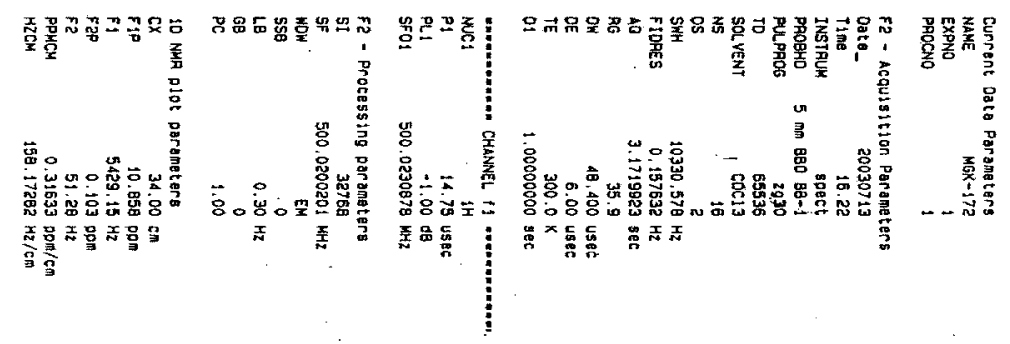



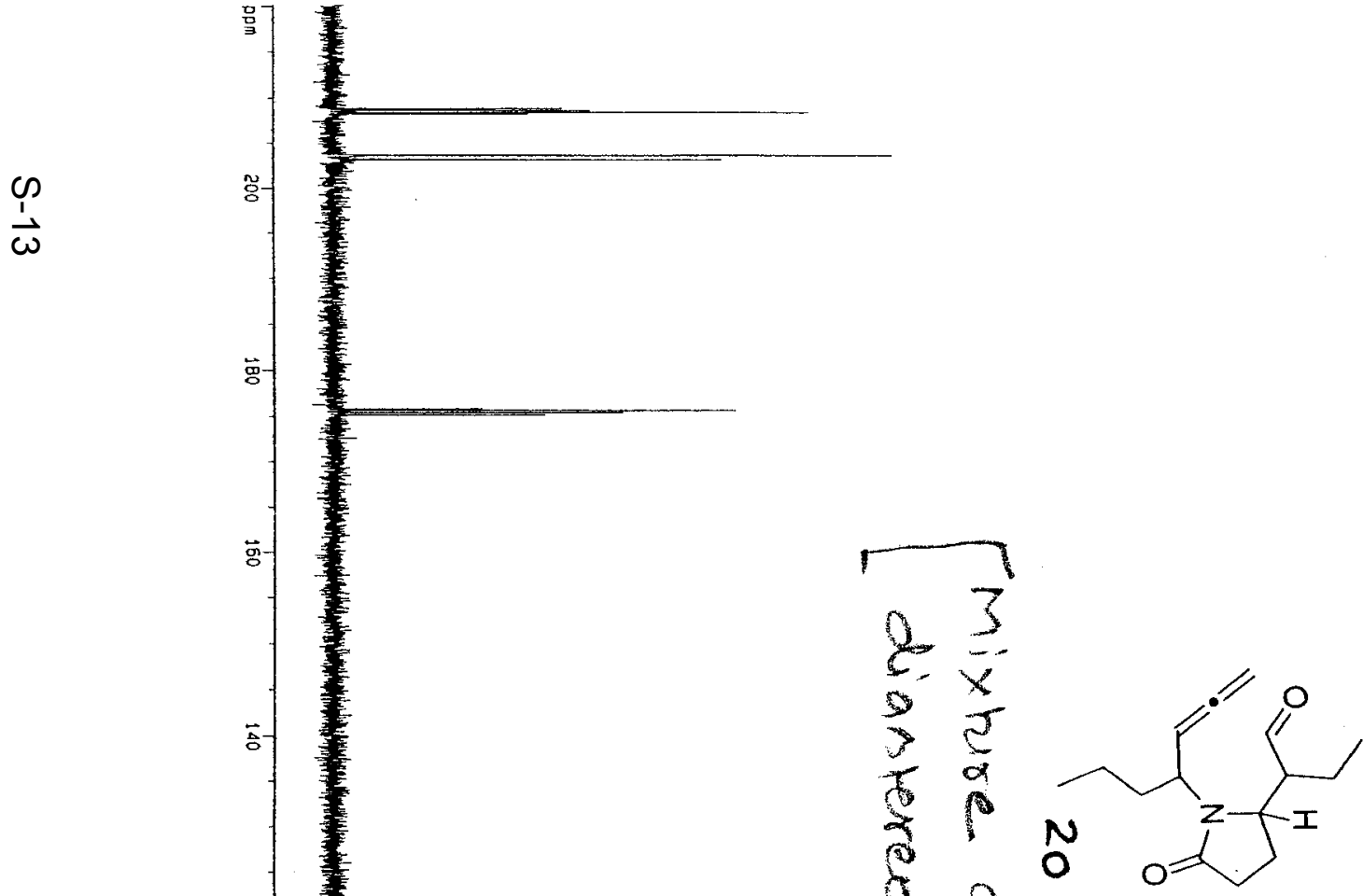

77.56

77.361

$\begin{array}{r}77.107 \\ -77.071 \\ \hline\end{array}$ 

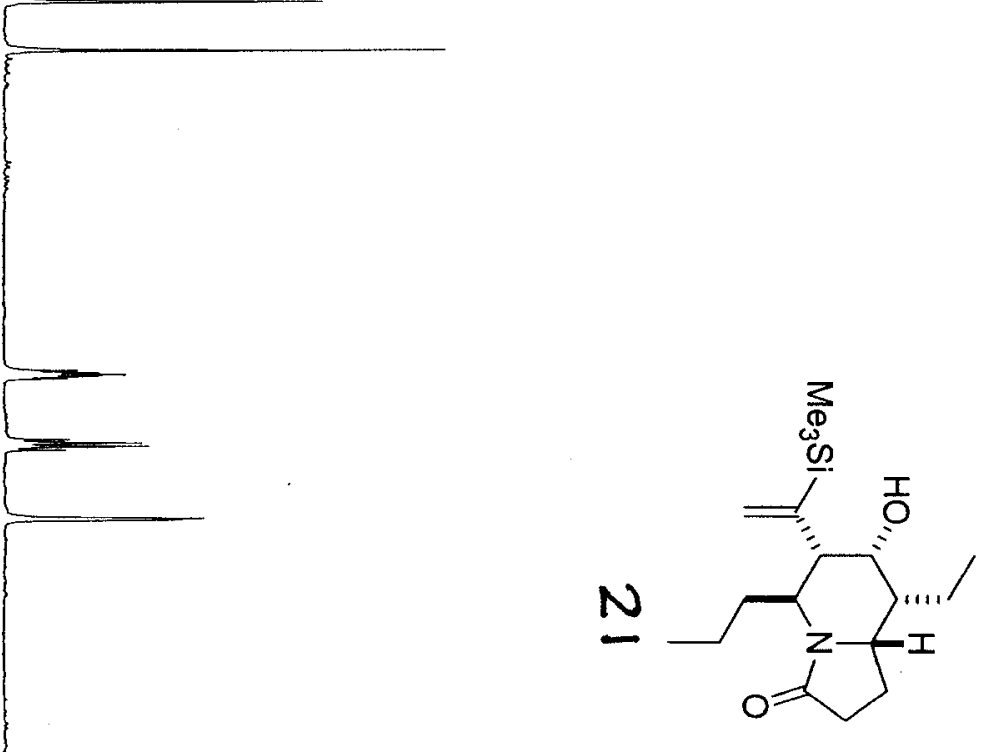

$-6.02018$

$10.924=$

11.540

12.330

8.724

$\frac{8.724}{23.095}$

23.045

$12.930=$

32.233

$\frac{35.497}{42.495}$

42.495

$\overline{97.209}$

2.209

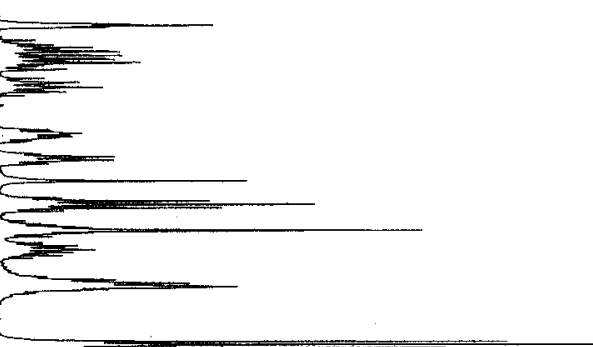

4.32236
-4.31360
-4.31059
-4.30306
-4.29527
4.28356

3.99988

$-3.98295$

$-3.96865$

$-3.95170$

$-3.63057$

$-3.62702$

$-3.62350$

$-2.46763$

. 34000

$-2.34082$

$-2.33667$

- 2.31949 fif-2.30052 Iff -2.28749 $-2.26239$

$-1.84818$

1.84818
1.83173

$\Gamma^{1.83173}$

$r^{-1.73635}$

$\iint^{-1.64464}$ $\sqrt{-1.62962}$ 1.51049

1.26452

1.28452

1.26738
-1.26142

-1.26142
-1.25275

牙 -1.24828

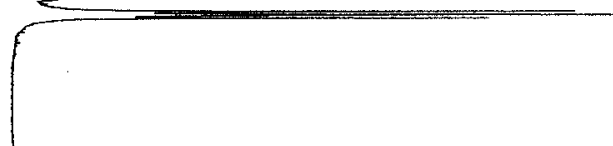

$L_{1.23909}$

$-0.99629$

0.98154

0.96675
-0.90253

0.90253
0.88782

$-0.88782$

$-0.15123$

0.11968

$-0.11129$

$-0.10509$

$-0.09859$

0.07382

$L_{0.06117}$

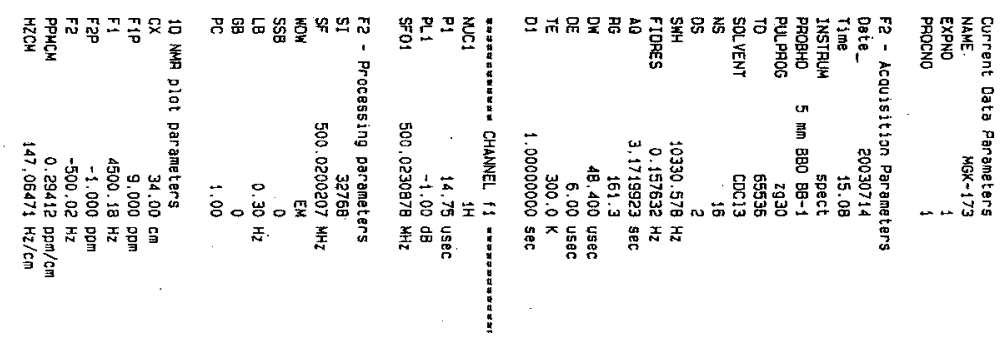


$\stackrel{\varphi}{\overrightarrow{1}}$

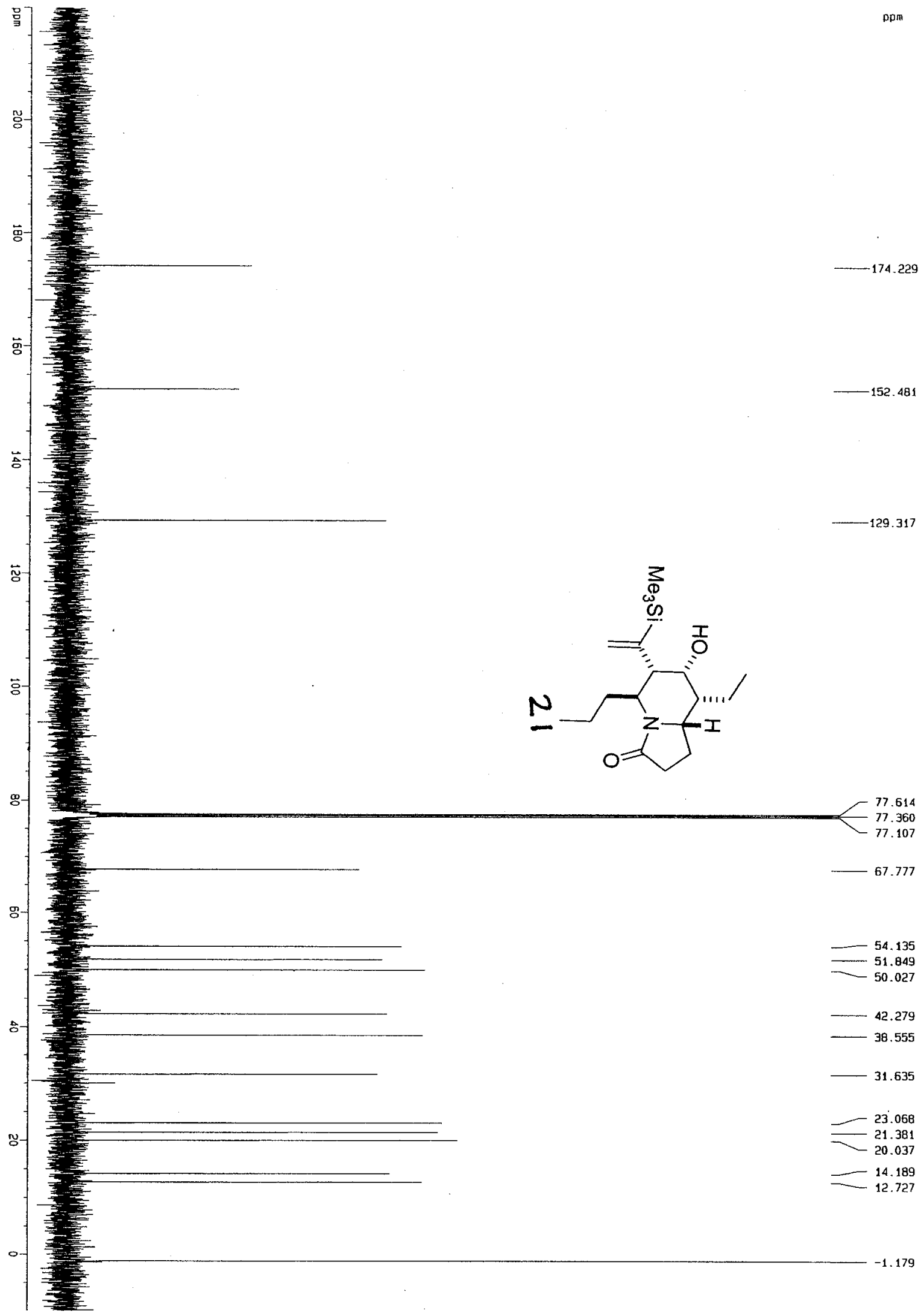

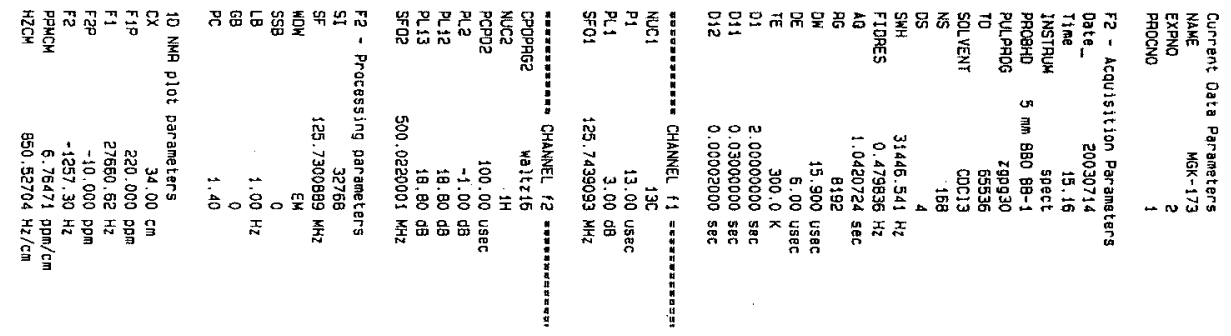




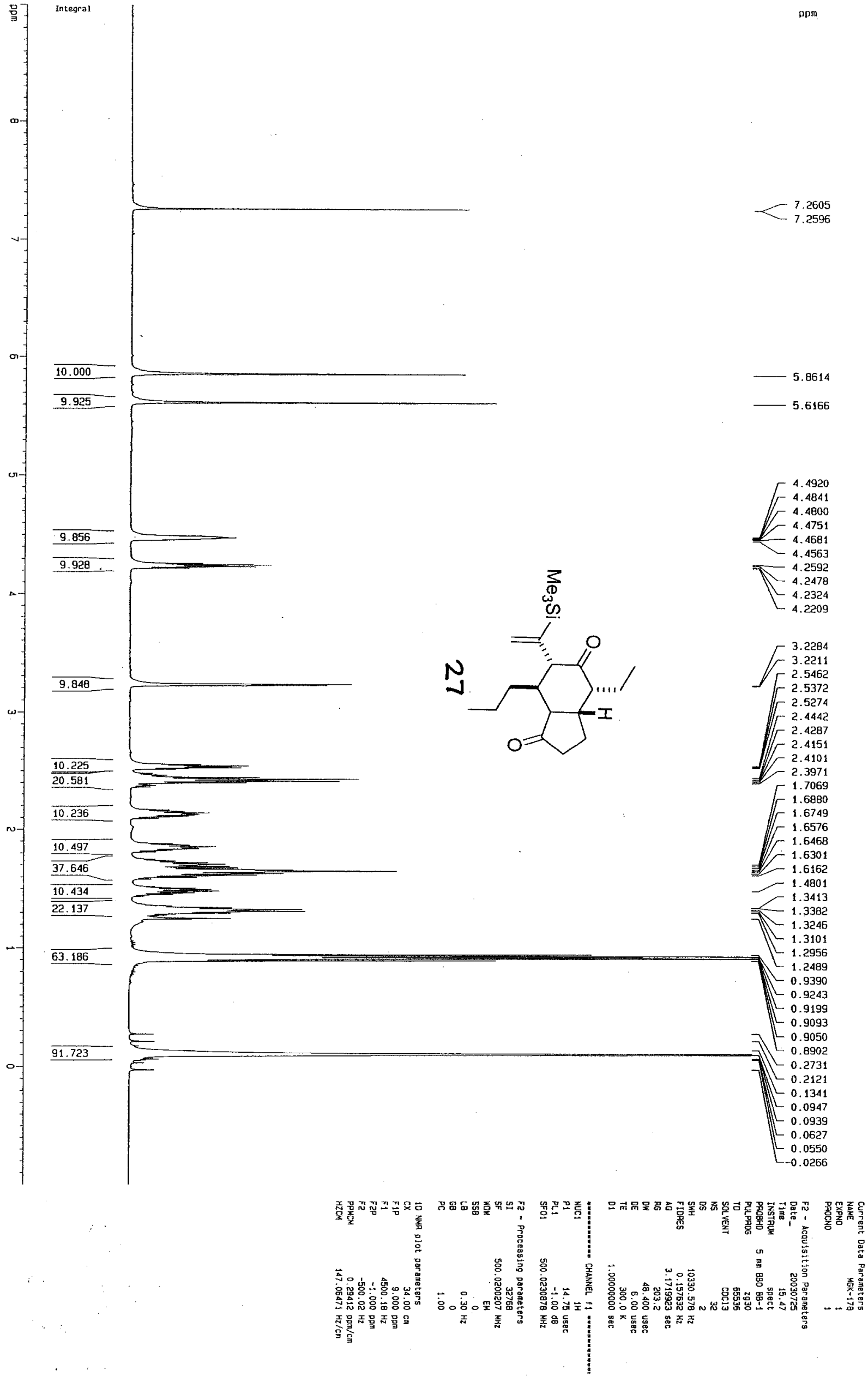



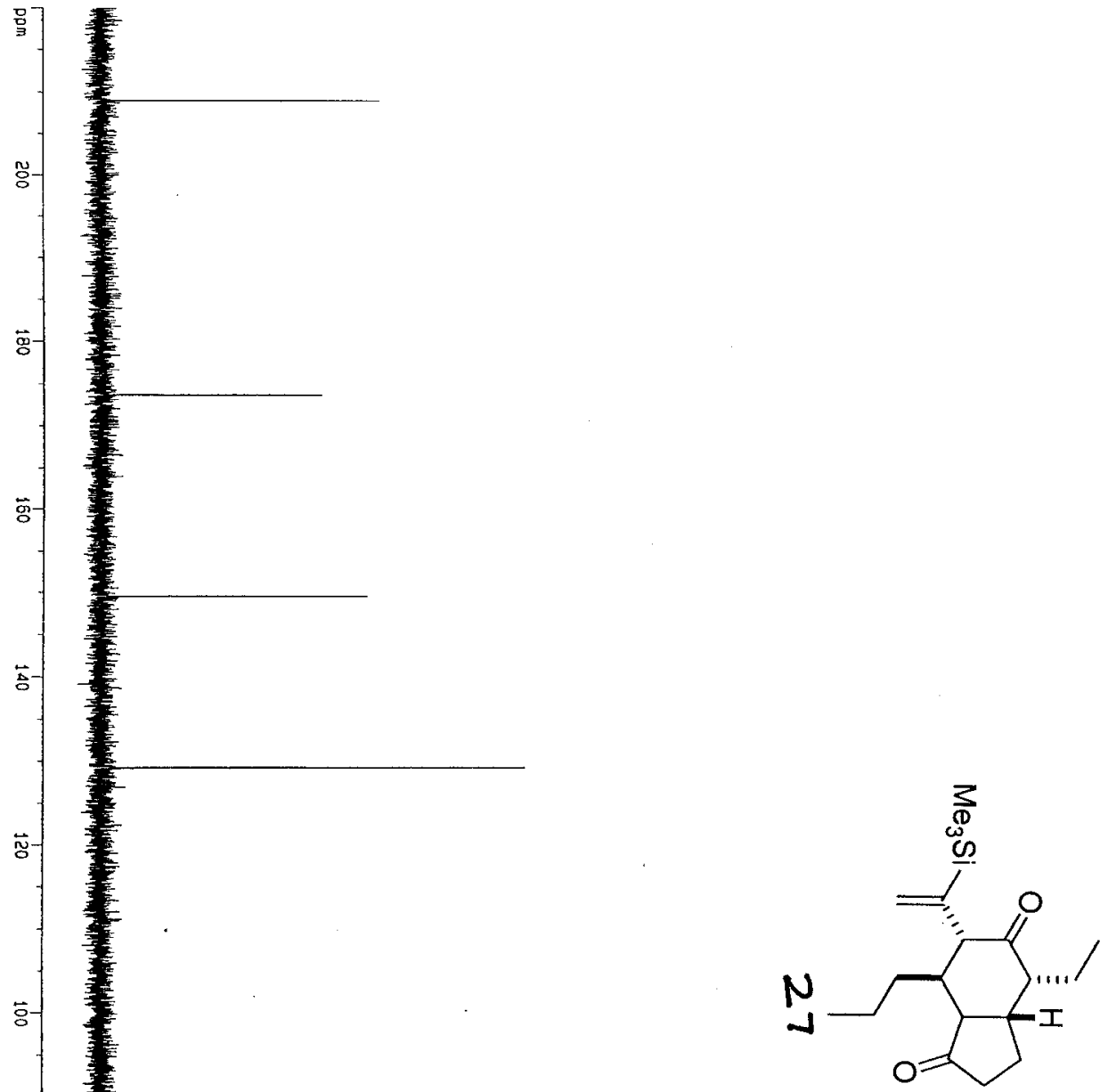

g

ก

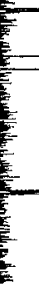

6.624 -31.221
$=\quad 30.042$ - 21.498

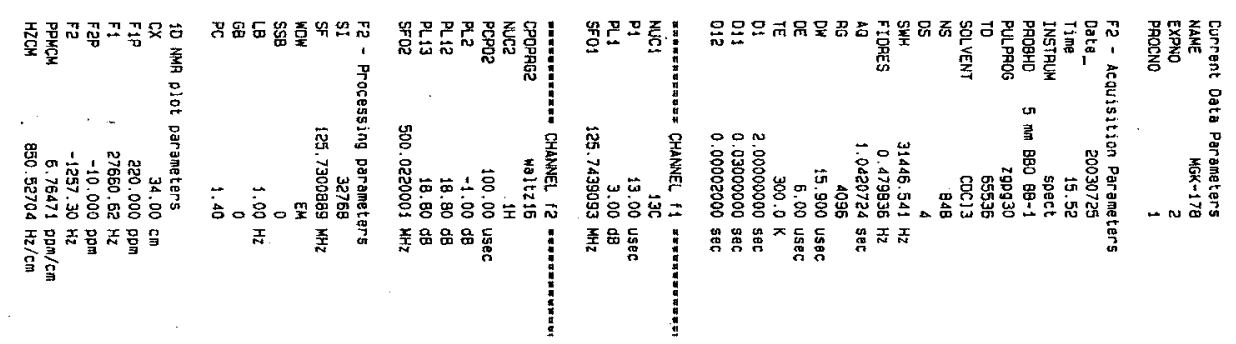




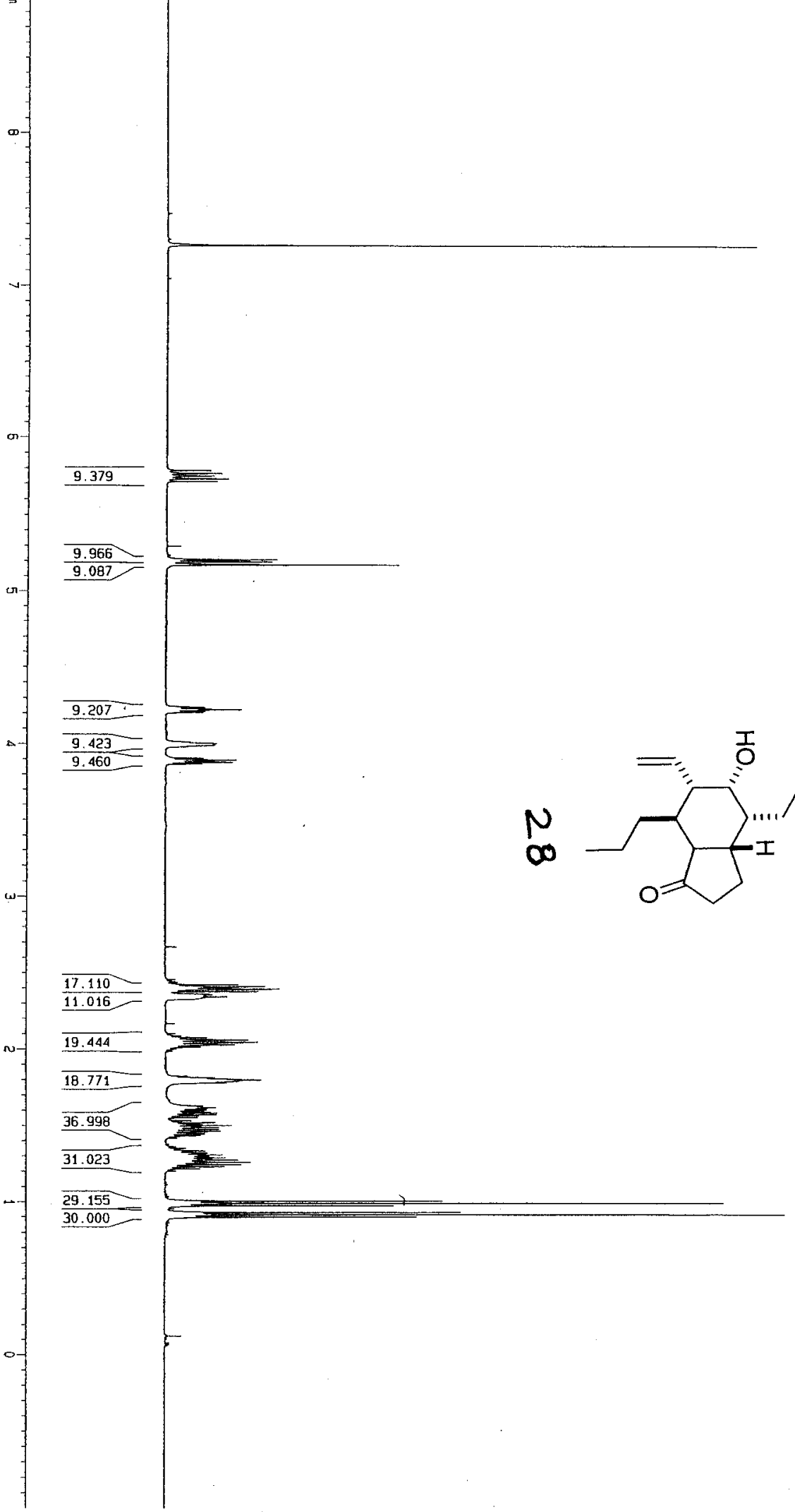

\subsection{9} 5.76806 5.76475 $-5.75203$ $-5.74720$ $-5.73417$ $-5.73093$ -5.20924 $\Gamma^{5.20924}$ 5.20640
-5.20415 5.20415
-5.19209 -5.17211
-5.17070 $-5.17070$ 4.23997 4.23523 -4. 22729 4.22233 -4.21733 -4.20944 $-3.99772$ $\begin{array}{r}-3.99004 \\ \hline\end{array}$ -3.90709 -3.90709
-3.89543 -3.89543
-3.89167 $-3.88372$ $-3.87977$

$-3.06822$ 2.42118 $-2.40940$ $-2.40302$ 2. 39206 $-2.38898$ $-3.3889$ . $-2.05799$ $\begin{array}{r}-2.04487 \\ -2.03940 \\ \hline\end{array}$ -2.03940
-2.02658 -2.02658
-1.79677 $-1.78843$

$-1.77920$

1. 49951

30522

1.28549

-1.27069
-1.25601

$\left[\begin{array}{l}-25601 \\ -1.24164\end{array}\right.$

$-1.24164$

$-1.22740$

$-1.00272$

0.98779
-0.97283

$L_{-0.92676}$

$-0.91211$

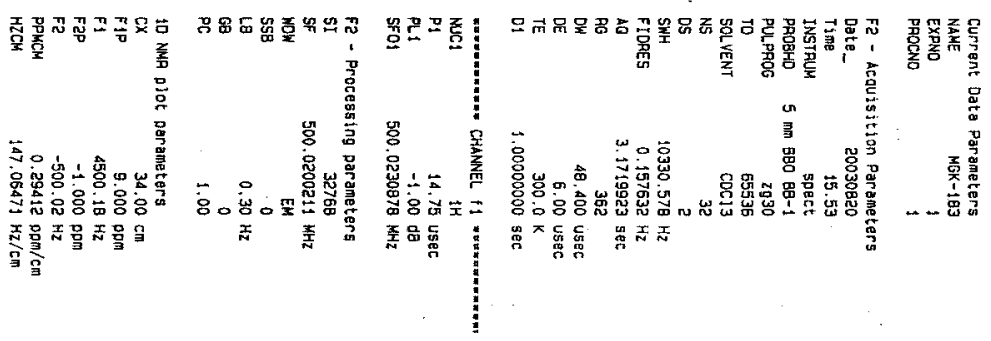




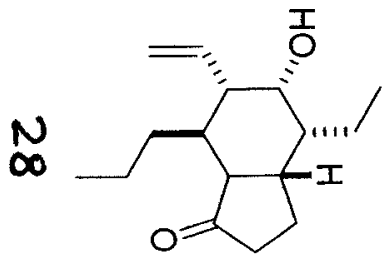


N
N

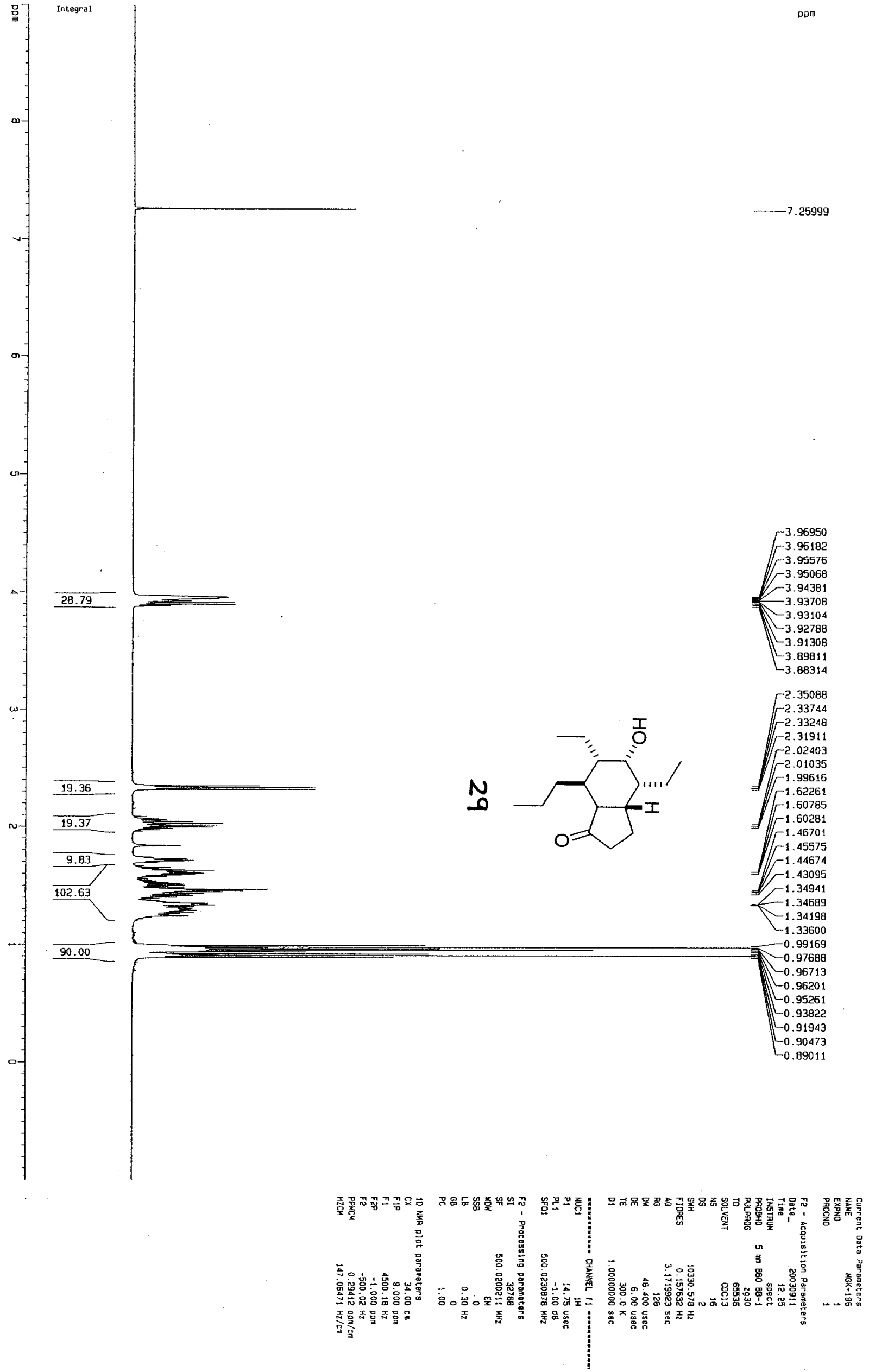




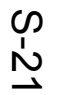

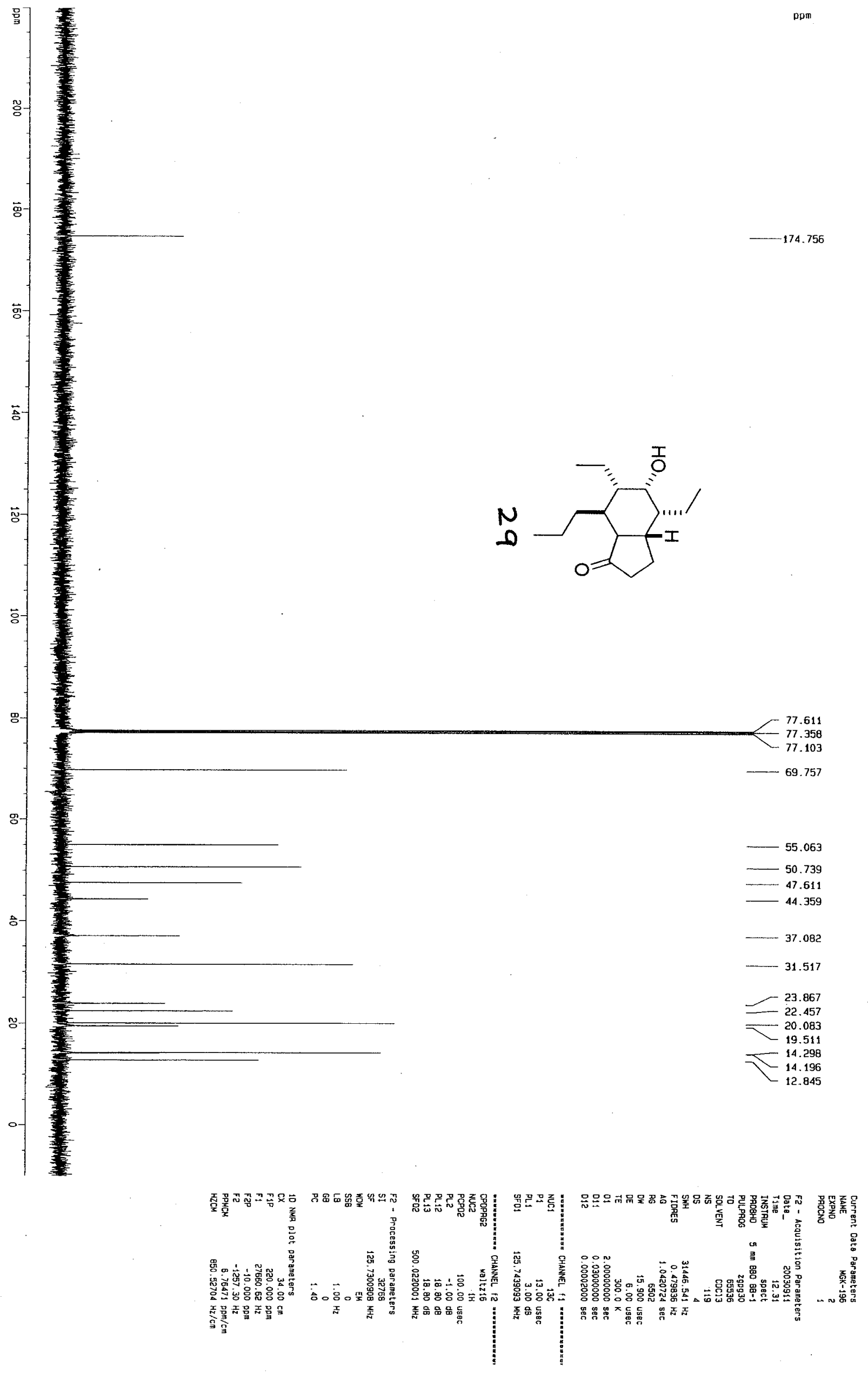


N
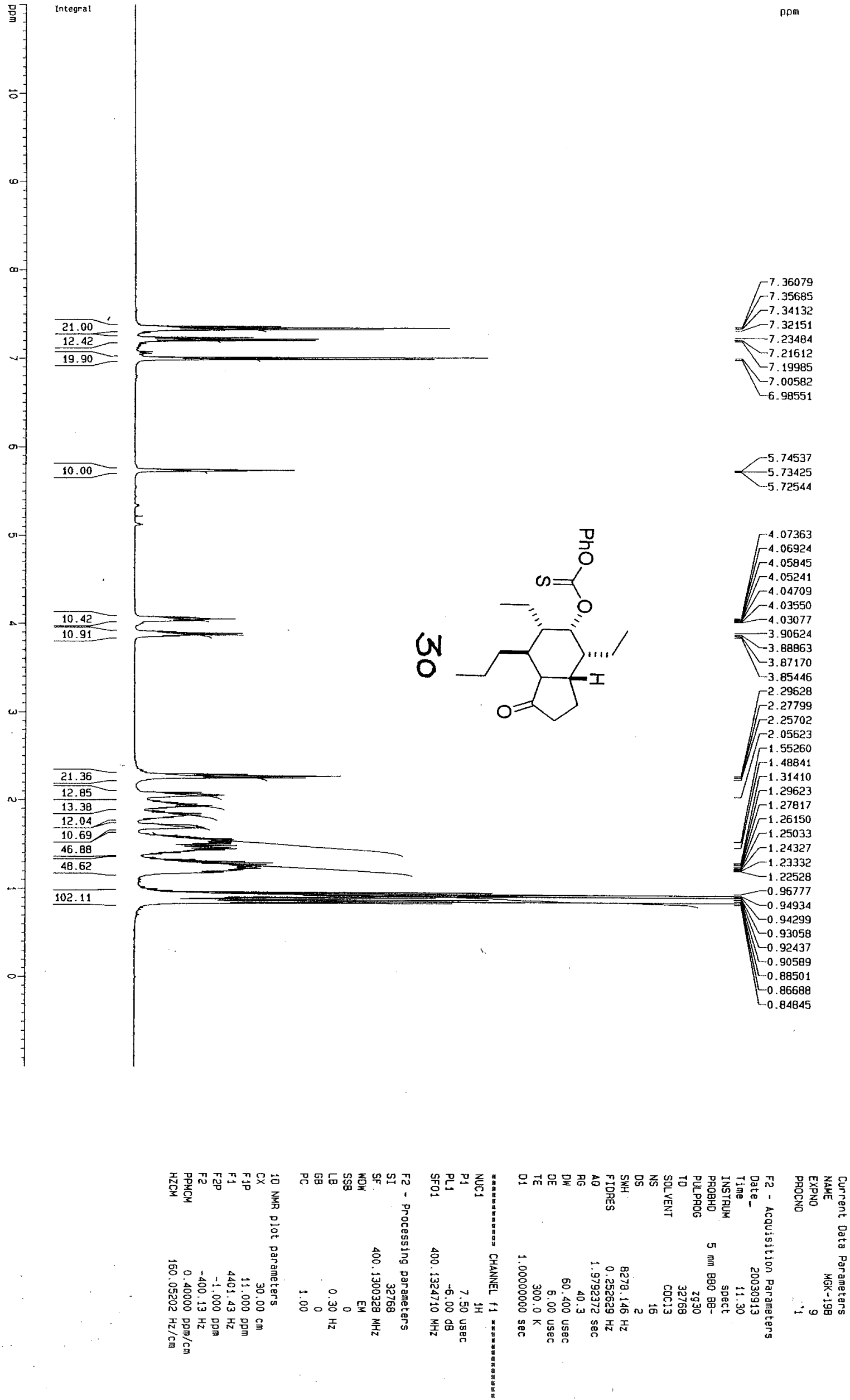

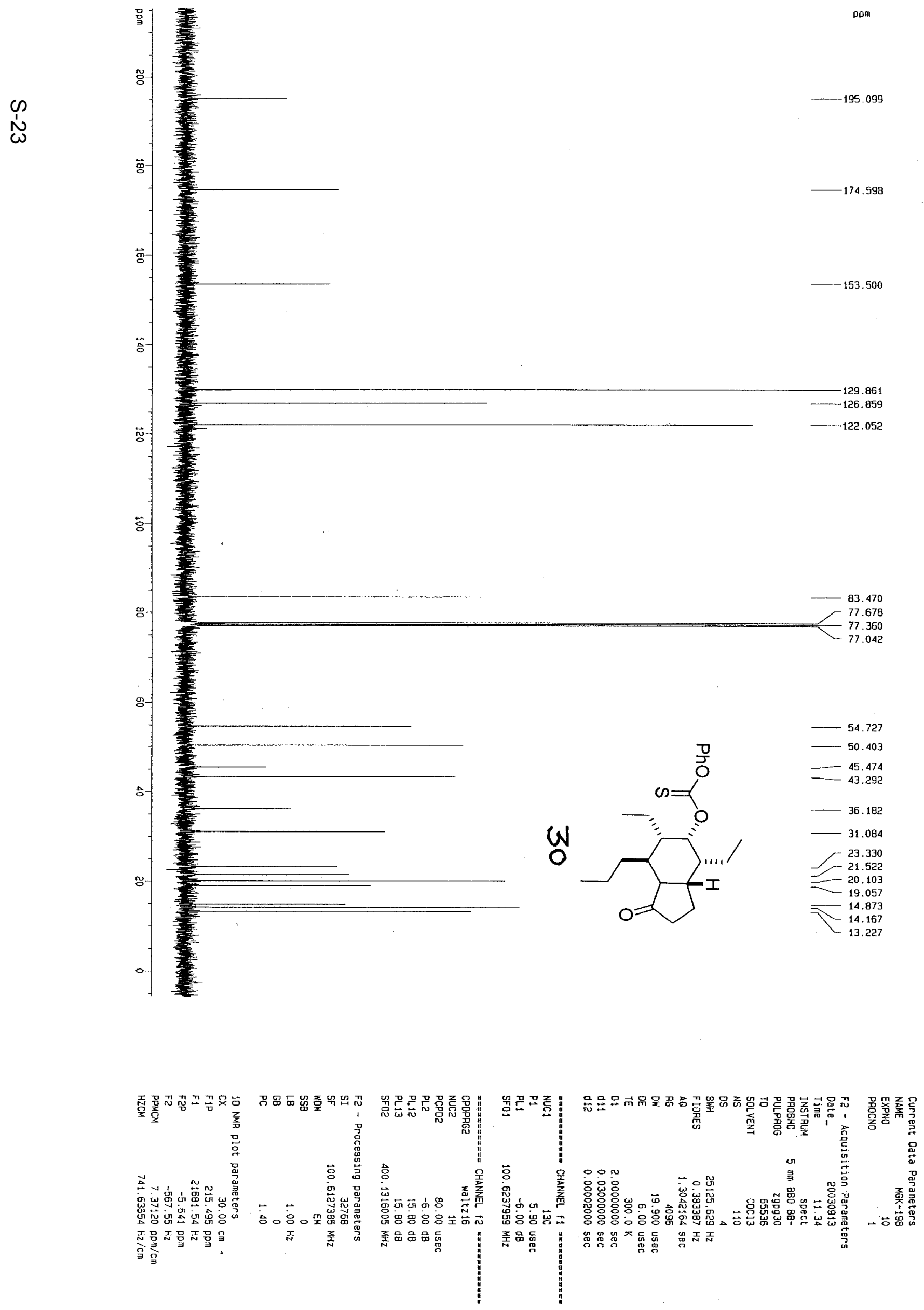

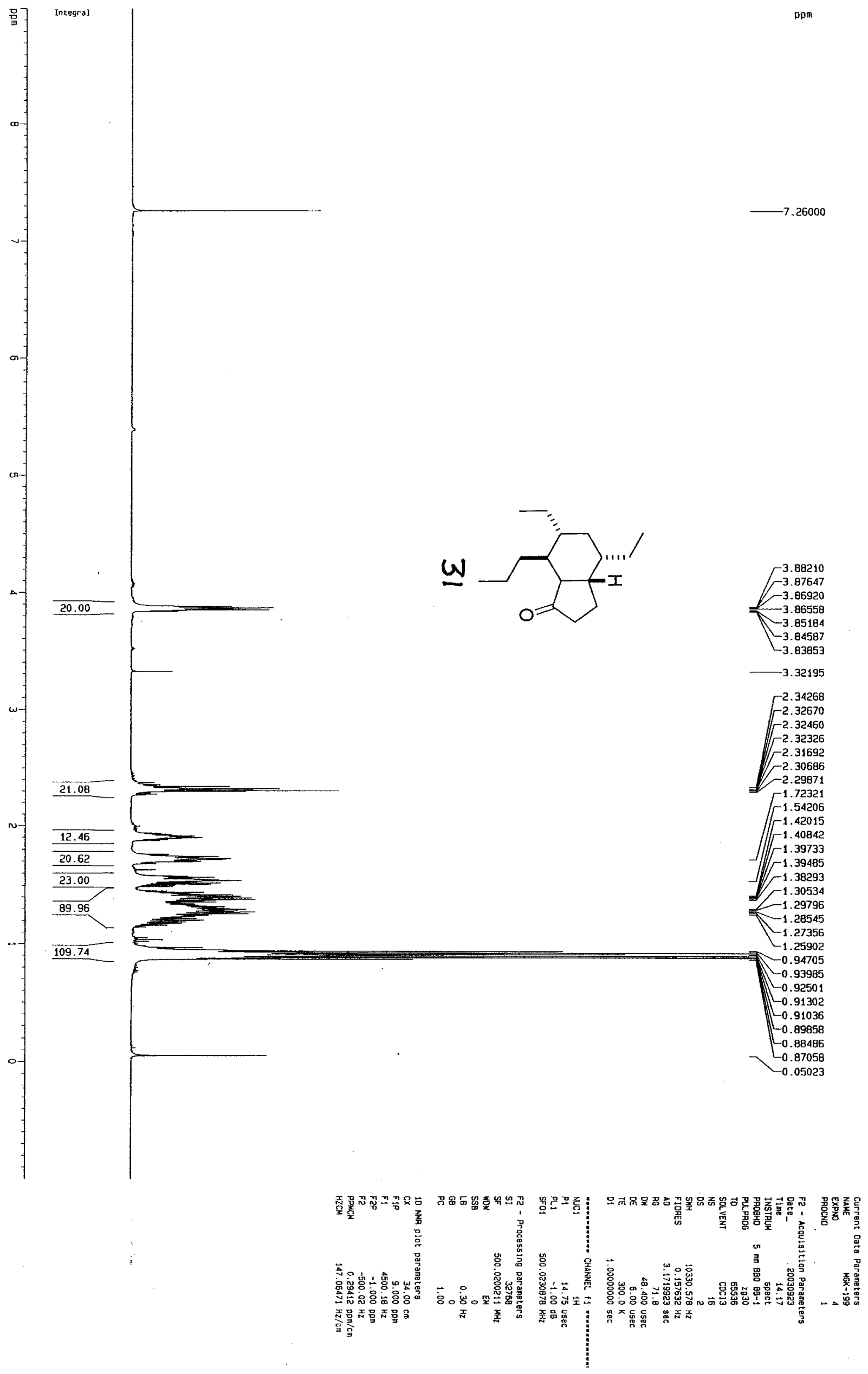


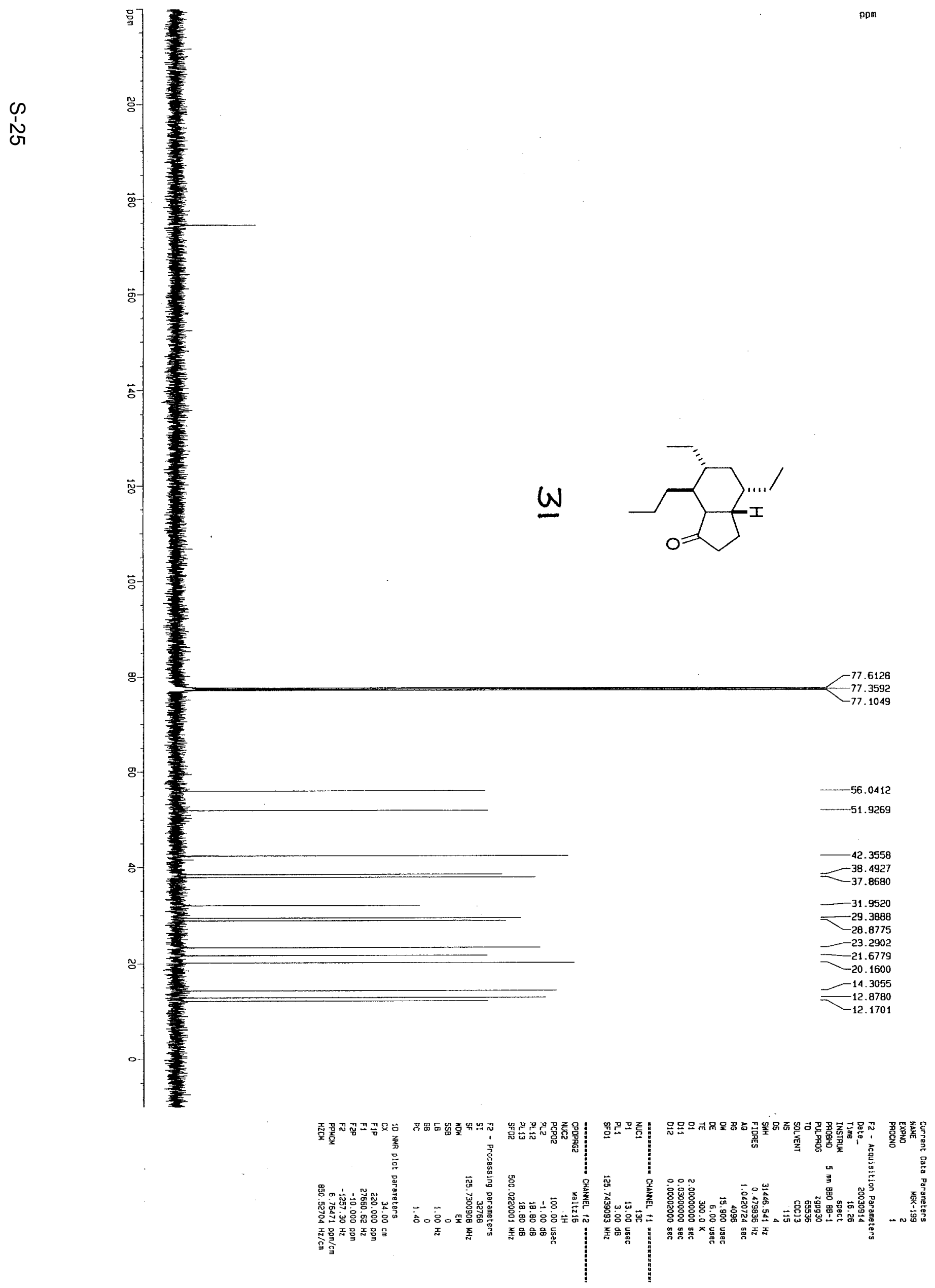


N

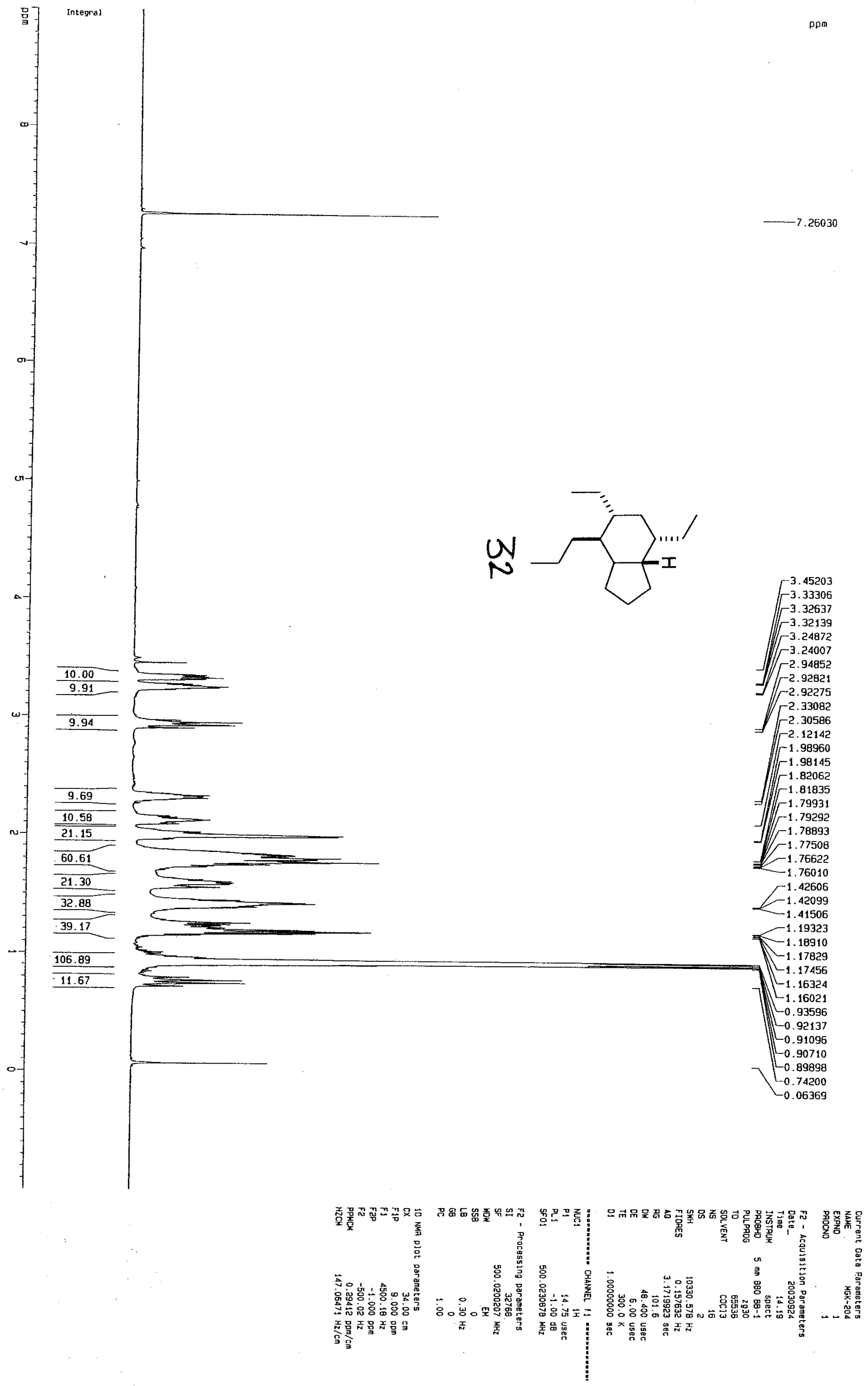




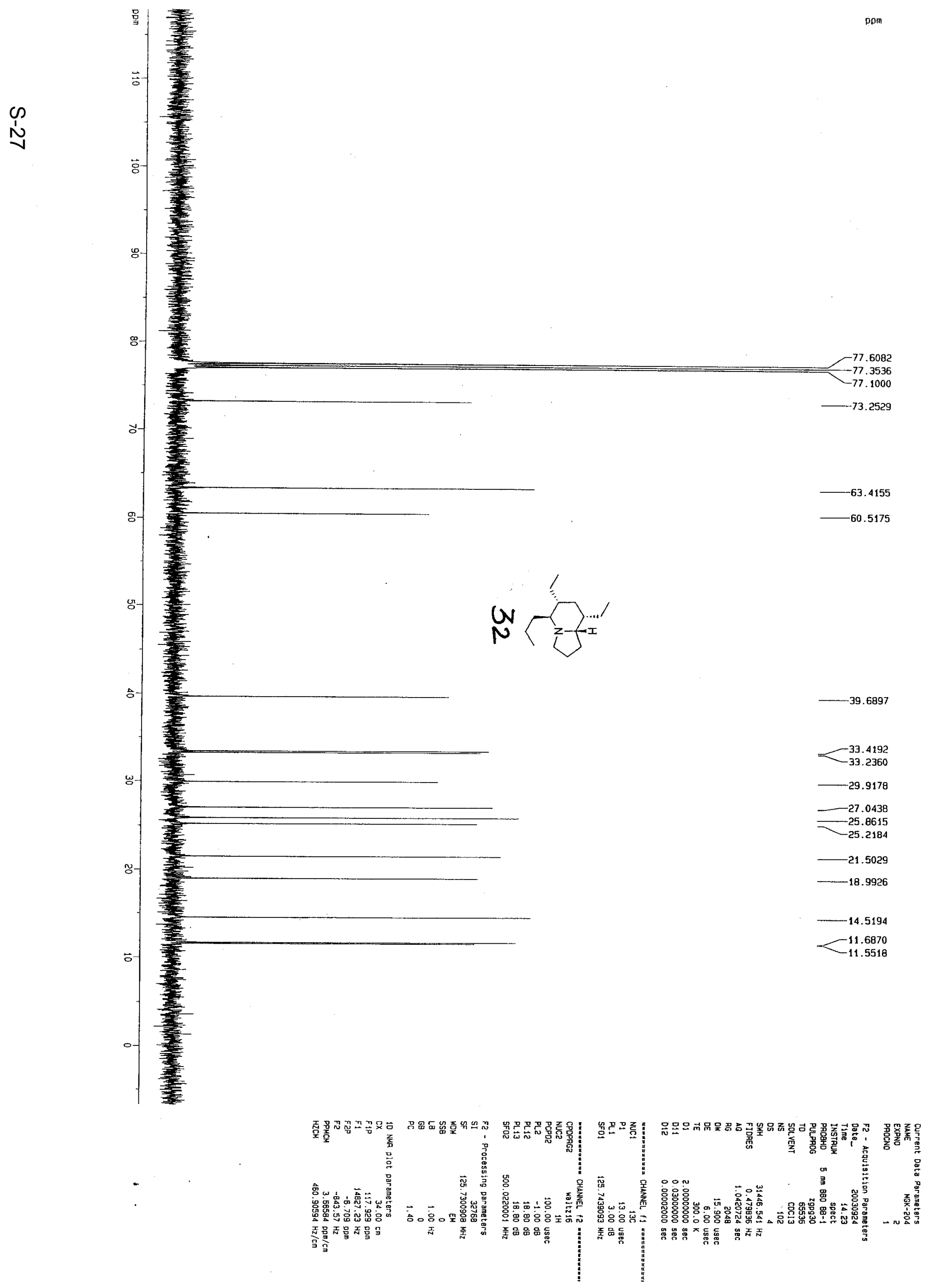




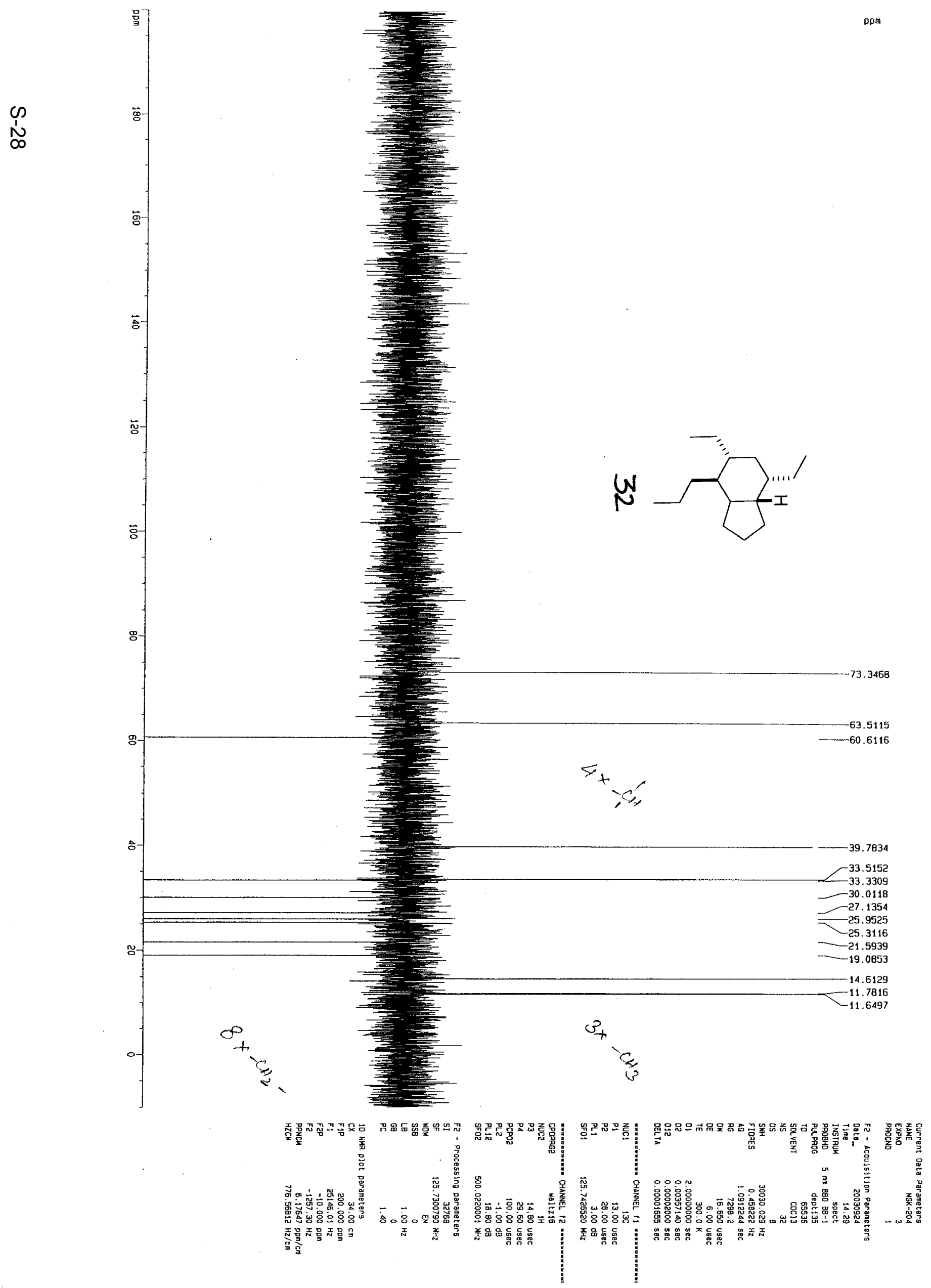


TIMETAELE STOP

N

Runt 455 Nov 11,2003 14:58:44

STGNAL FILE: MISTEHAL, EMC

HO RUH FEAKS STORED

* Run \# 456 HOV 11, $2003 \quad 15: 48: 11$

START

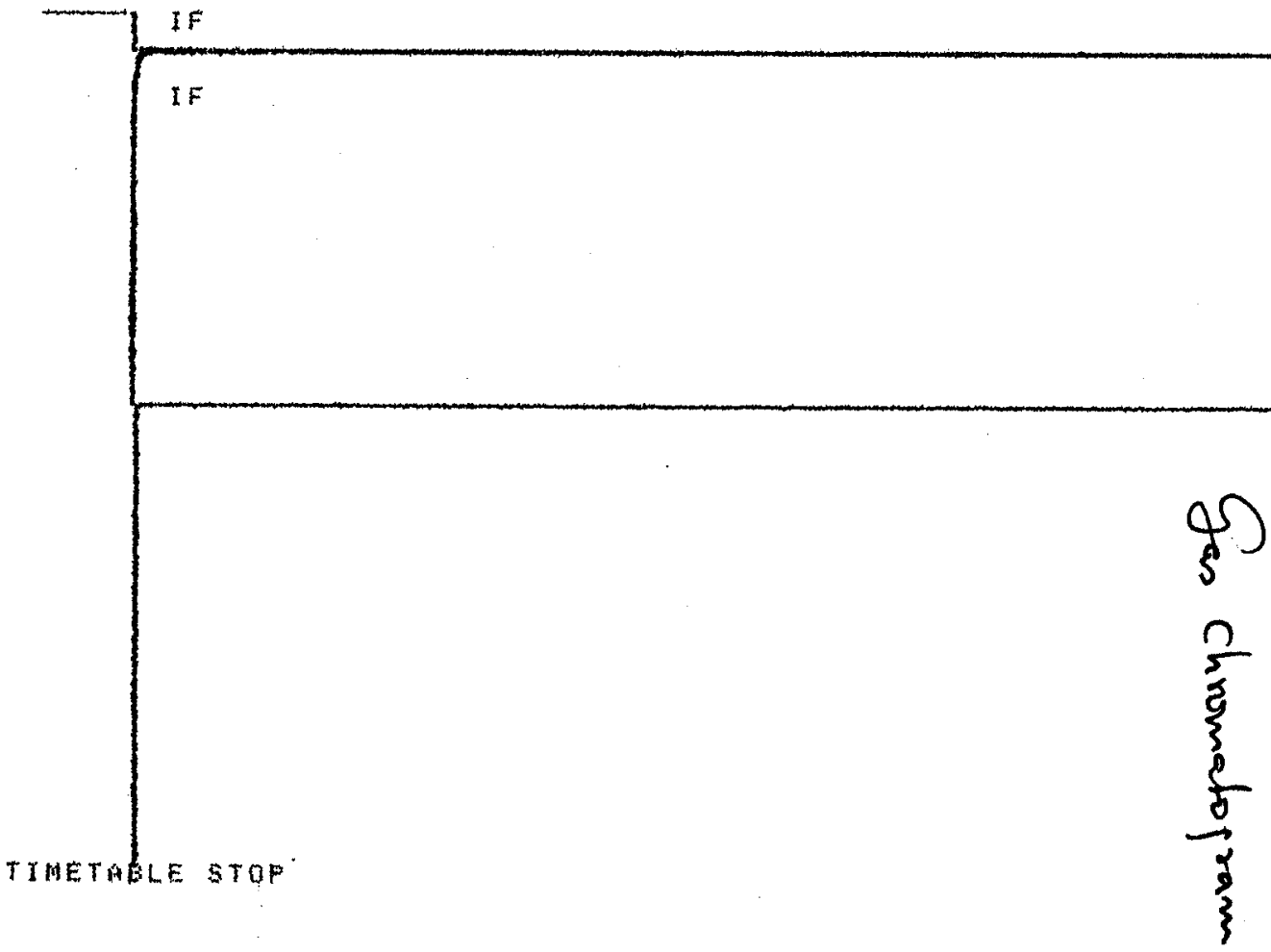

t10stna signal file MISIGNAL BHC

RUNA 456 MOY $11 * 2693 \quad 15 \% 40: 11$

SIGHAL FILE: B: SIGNAL. BNC

AFEAY

\begin{tabular}{|c|c|c|c|c|}
\hline RT & AREA & TYPE & W1 ITH & $A R E A \%$ \\
\hline 20.553 & 141355 & $P \mathrm{~B}$ & .030 & 100.00000 \\
\hline
\end{tabular}

TOTAL AREA $=1413556$

MUL FACTOR=1.0000E+00

20.523

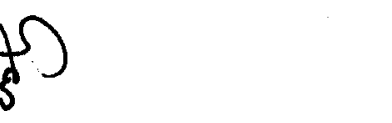

$$
3
$$
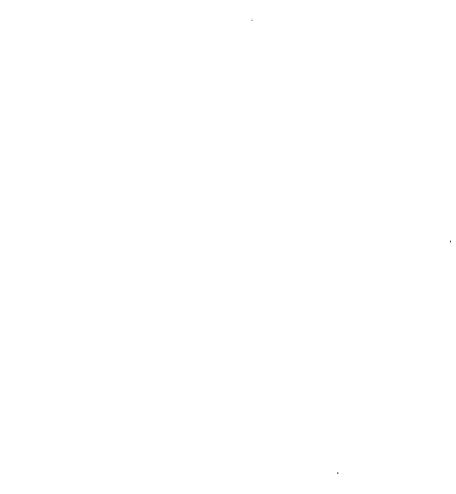


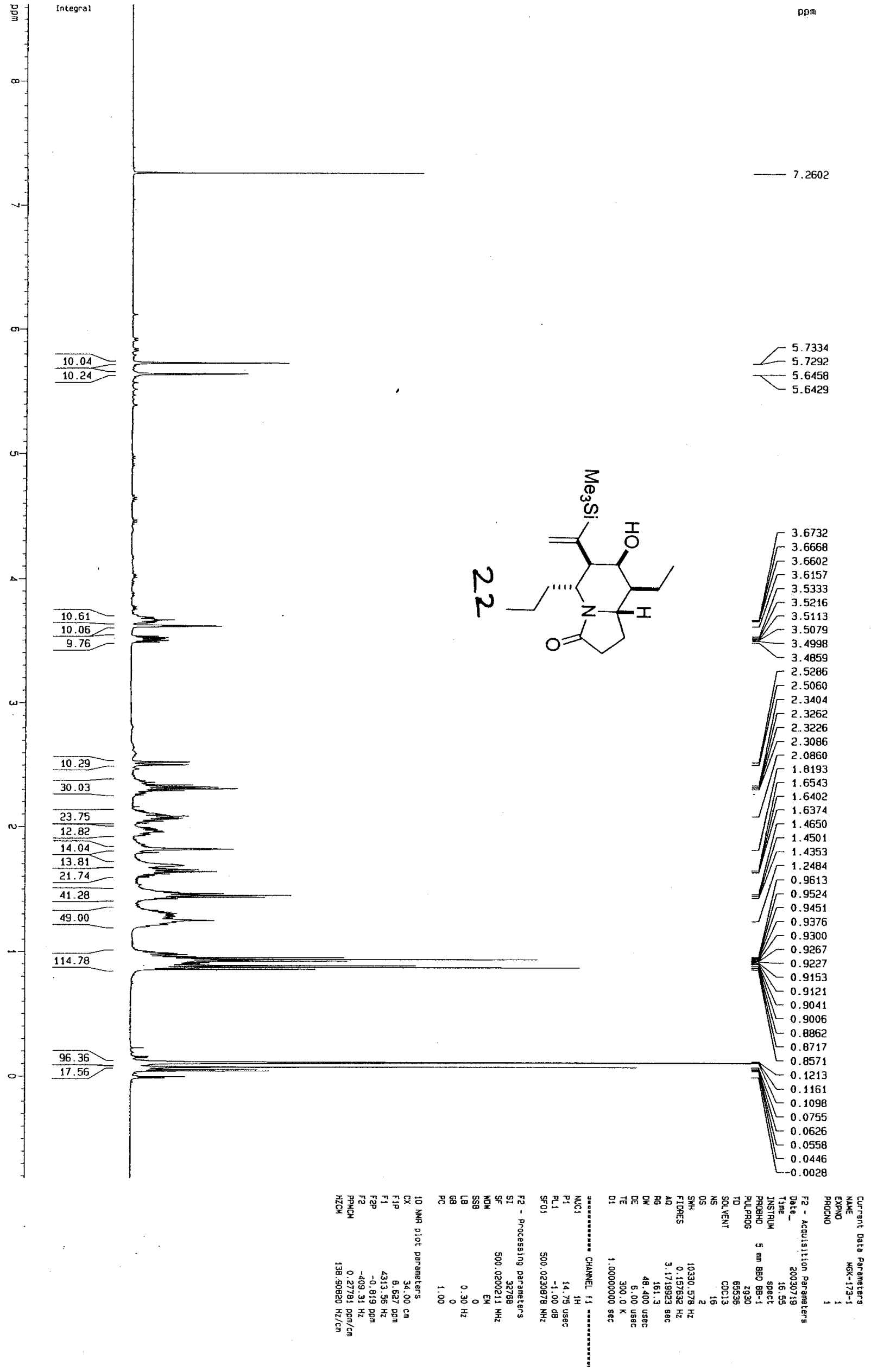




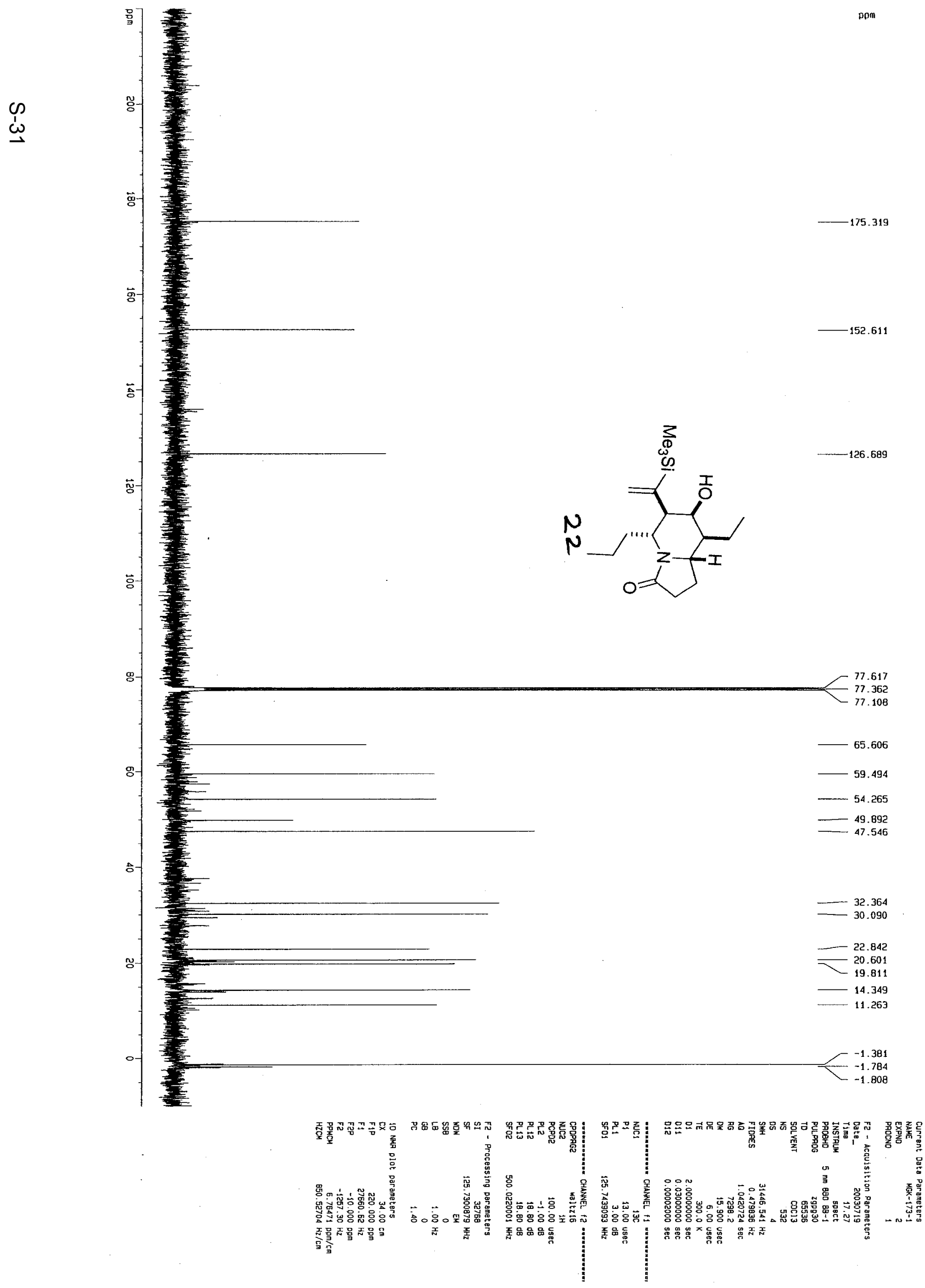


5.78616 5.22677

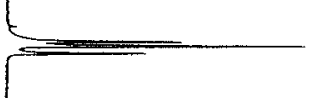

5. 22363

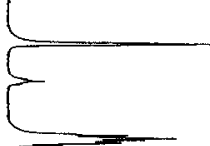

$\omega$

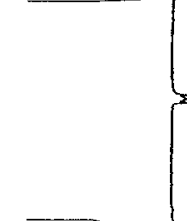

21.174

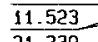

21.339

35.248

19.517

76.713

4.057

74.067
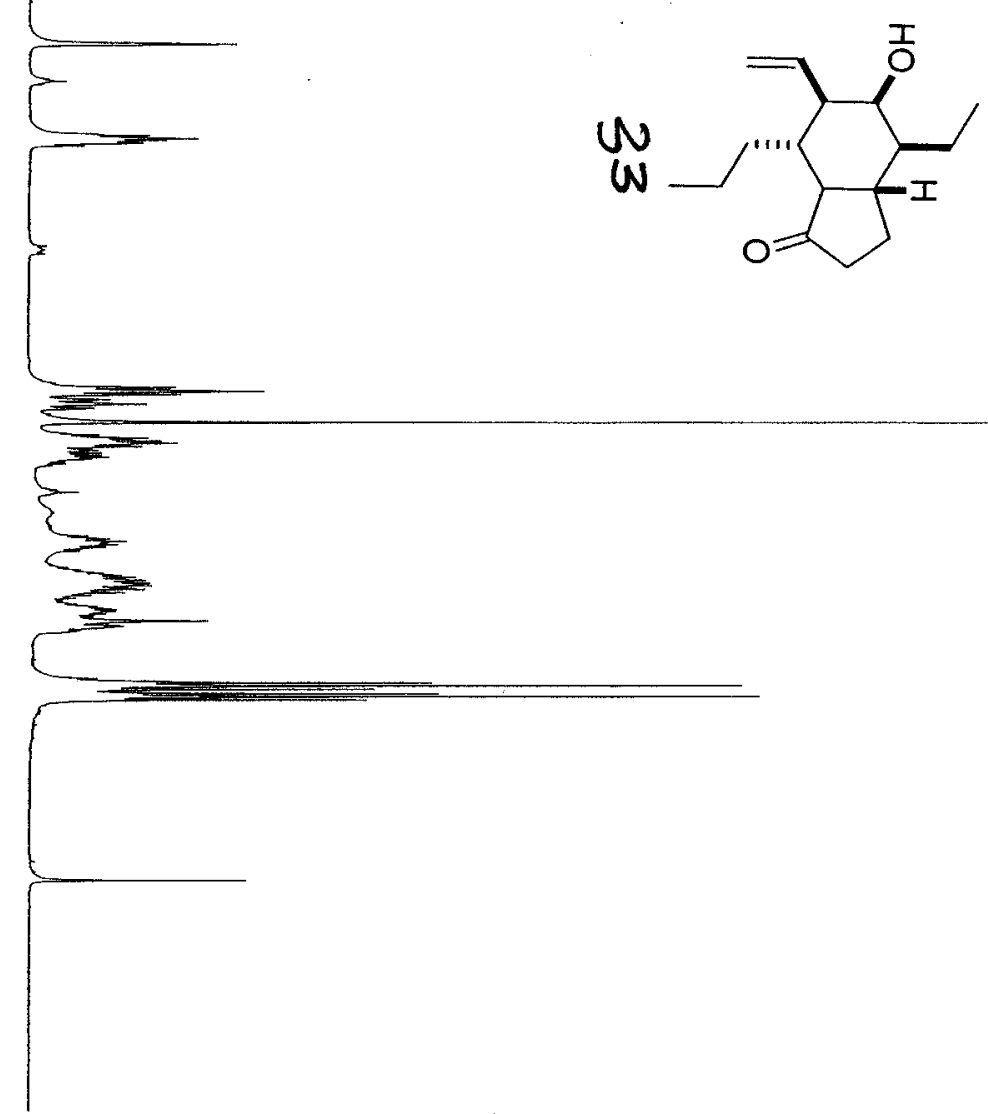

$-3.91652$

$-3.50254$

$-3.49505$

-3.48092
-3.47209

-3.47299
-3.46142

$-3.44755$

2.33128

$-2.31688$

$-2.31302$

$r^{-2.29924}$

r-25257

$\sqrt{-2.16632}$
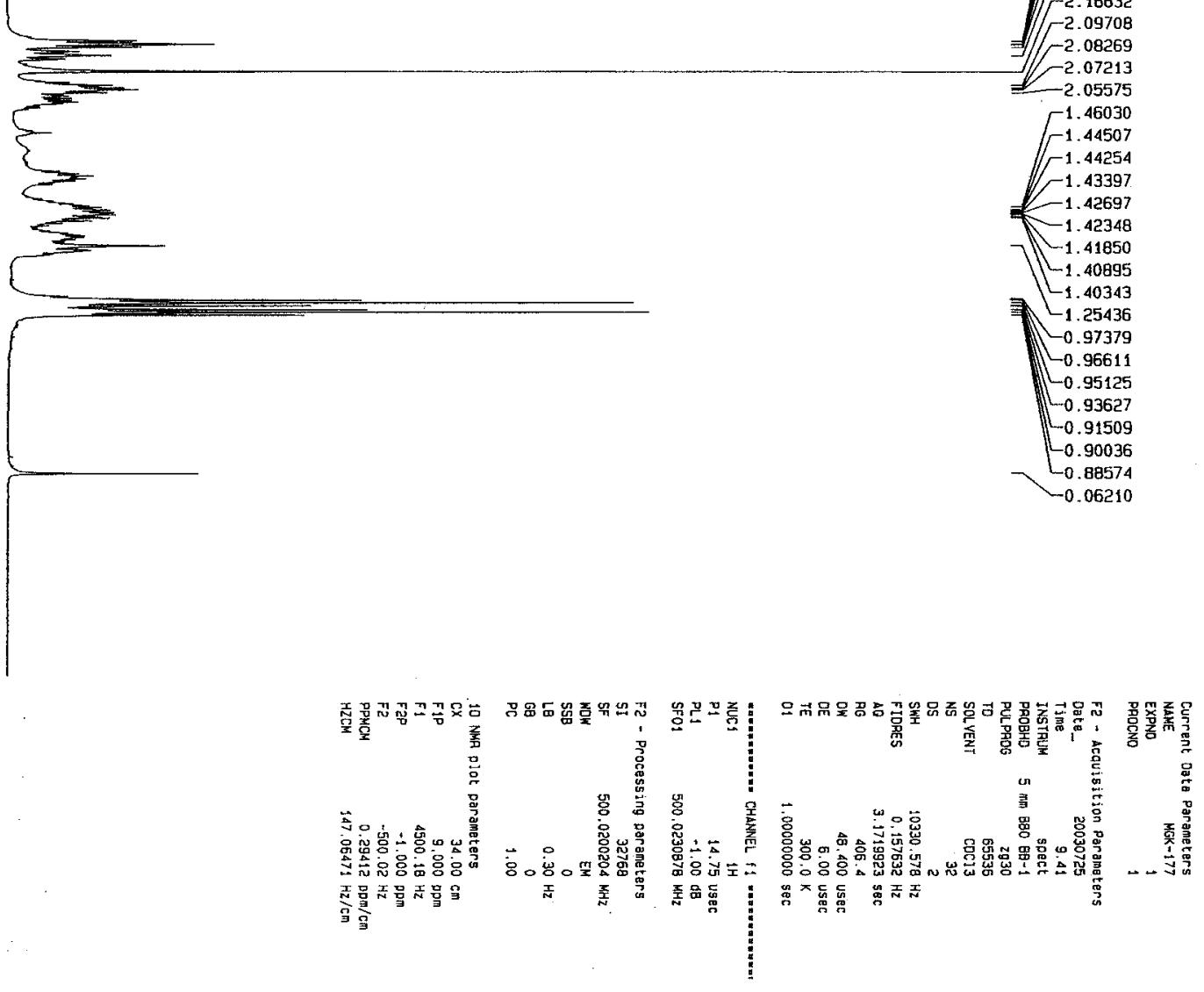
畧
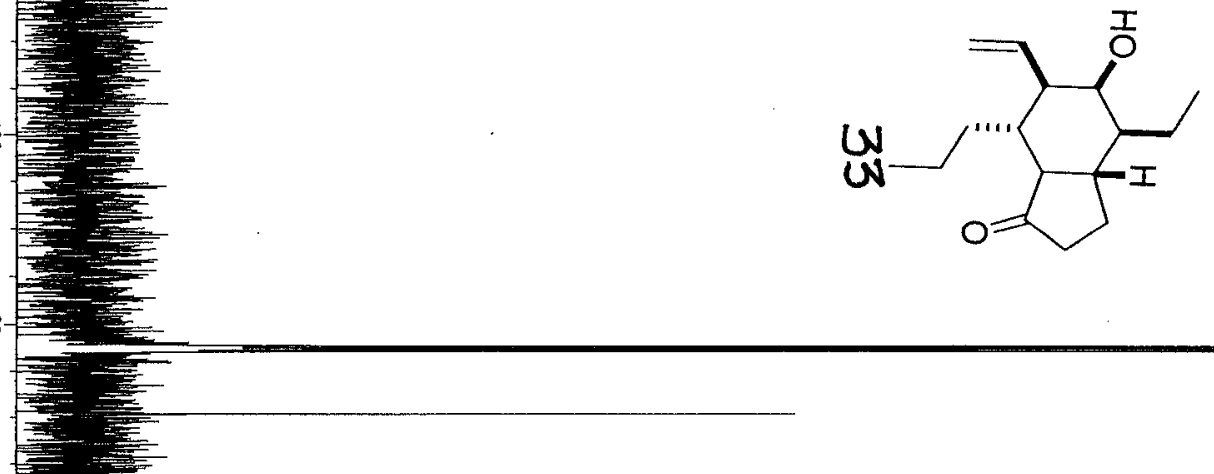

s

-

$=$

54.41

a

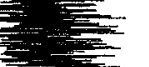

$\frac{5}{2}$

s-

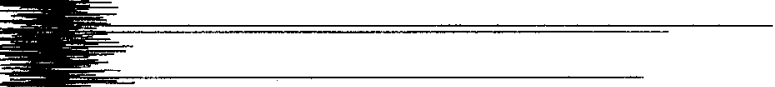

32.275

31.258

30.927

22.999

$=19.940$

$\checkmark 19.293$

- 14.476

$\circ$ 

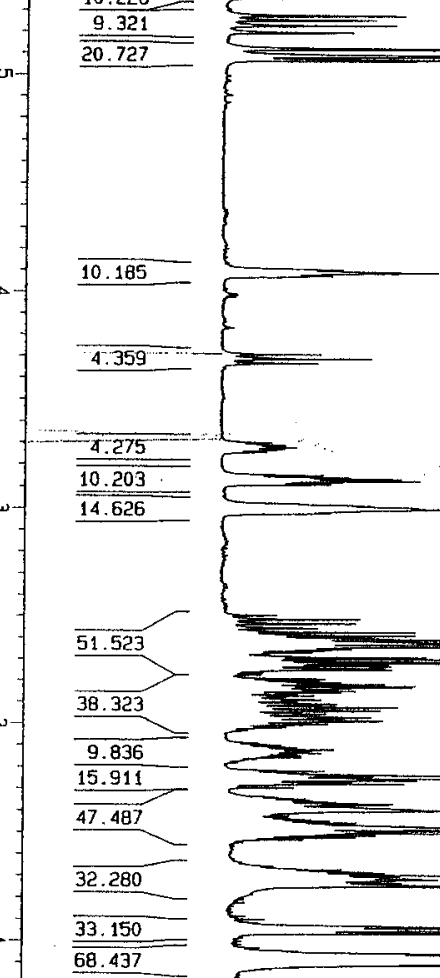

68.437
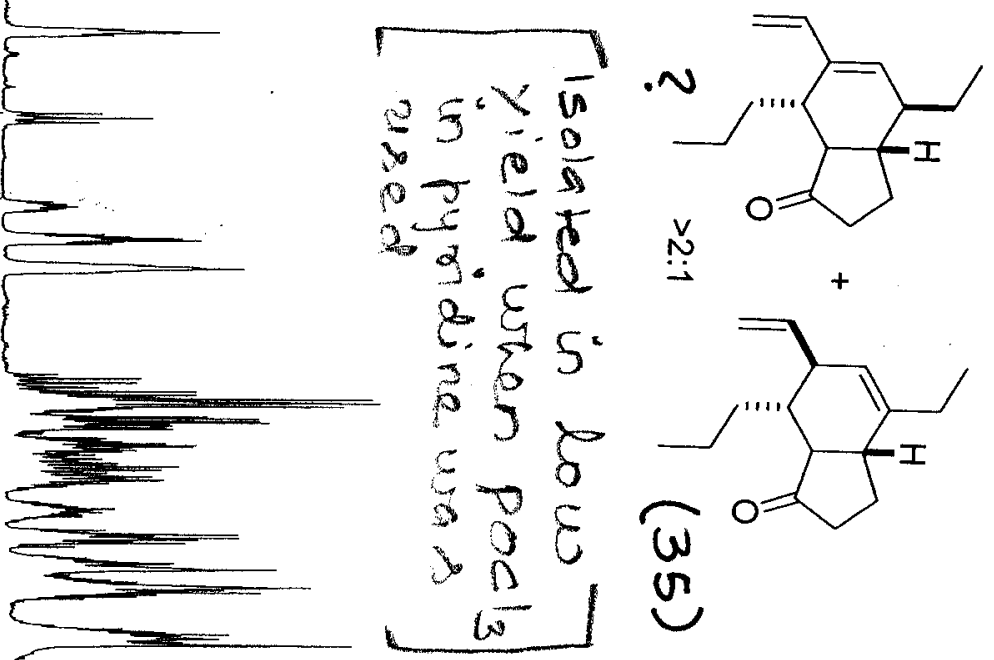

$\left[\begin{array}{r}5.60188 \\ -5.59992 \\ 5.58587 \\ -5.58169 \\ -5.56551 \\ -5.36767 \\ -5.36441 \\ -5.36121 \\ 5.35796\end{array}\right.$ $-5.12002$

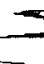
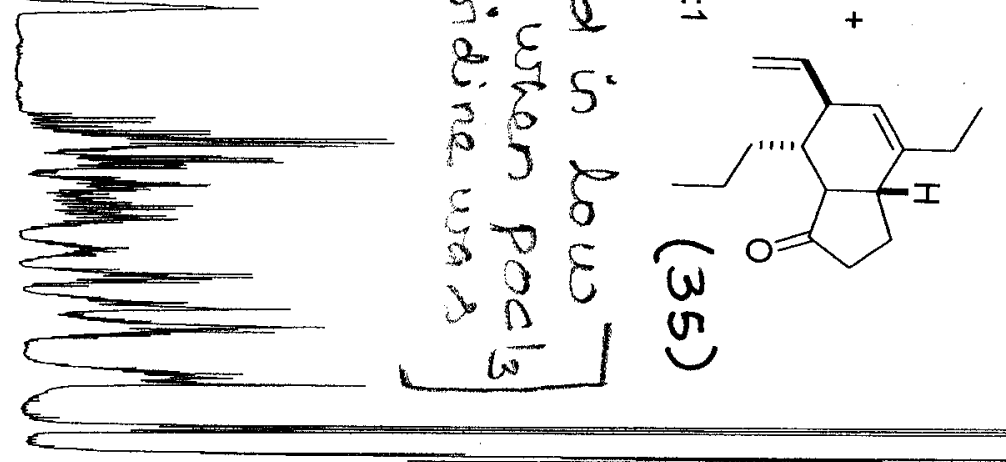

5.11460
5.08701
-5.08603
5.08368

5.08050

$-5.06704$

$-5.06599$

$-4.08574$

$-3.68518$

$\Gamma^{3.13392}$

-3.12466

$-3.00343$

$\int r^{3.903432}$

$\int / r^{2.99542}$

$\int \mathrm{r}^{-2.39308}$

- -3.37923

$-2.37578$

III -2.35921

-2.30154

IIf-2.29119

If -2.2830

$\left[\begin{array}{l}1.76337 \\ -1.74813\end{array}\right.$

$-1.74813$

$-1.5281$

$r_{-1.52276}^{1.52816}$

I -1.52276

1.51849

-1.49719

$-1.25383$

$-1.05792$

$-1.05305$
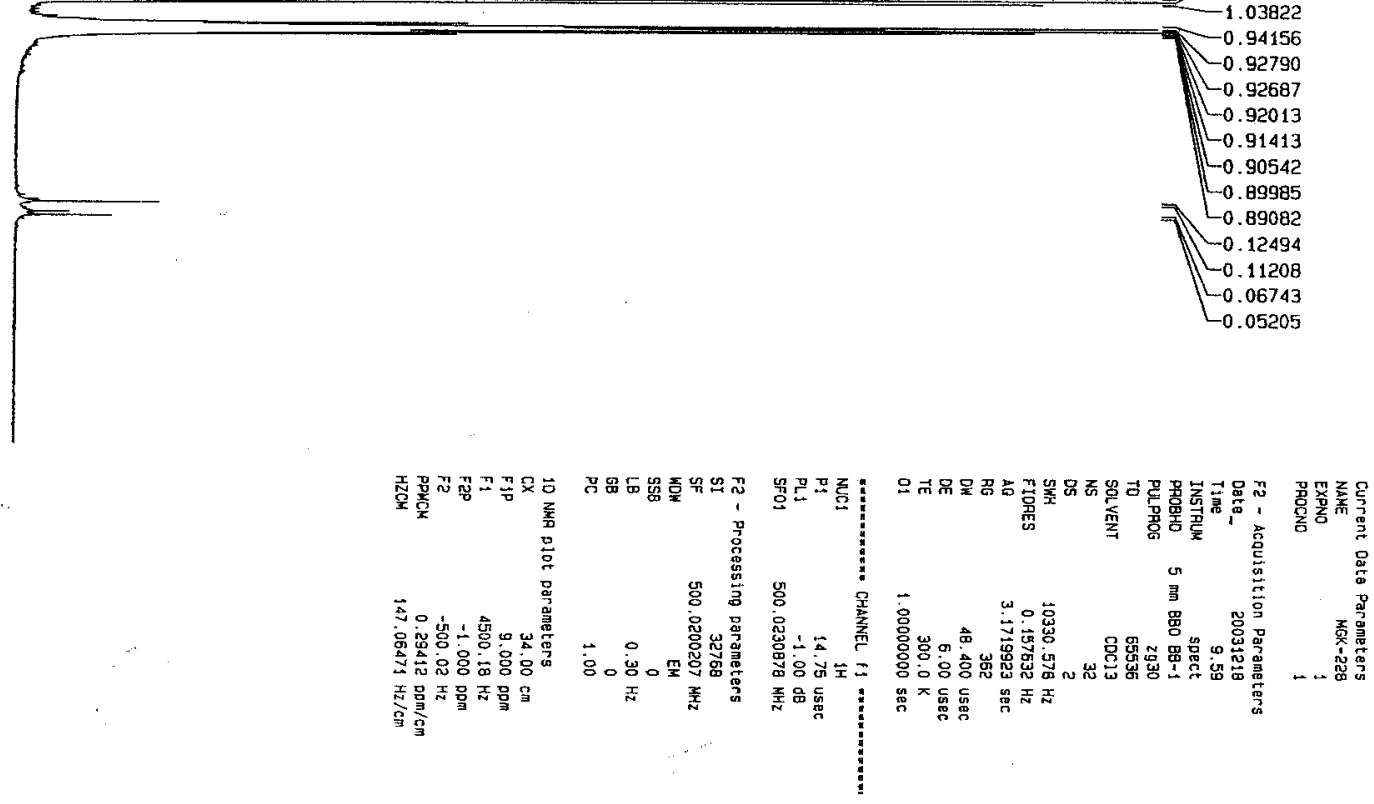
$\omega$
$\omega$
$\omega$
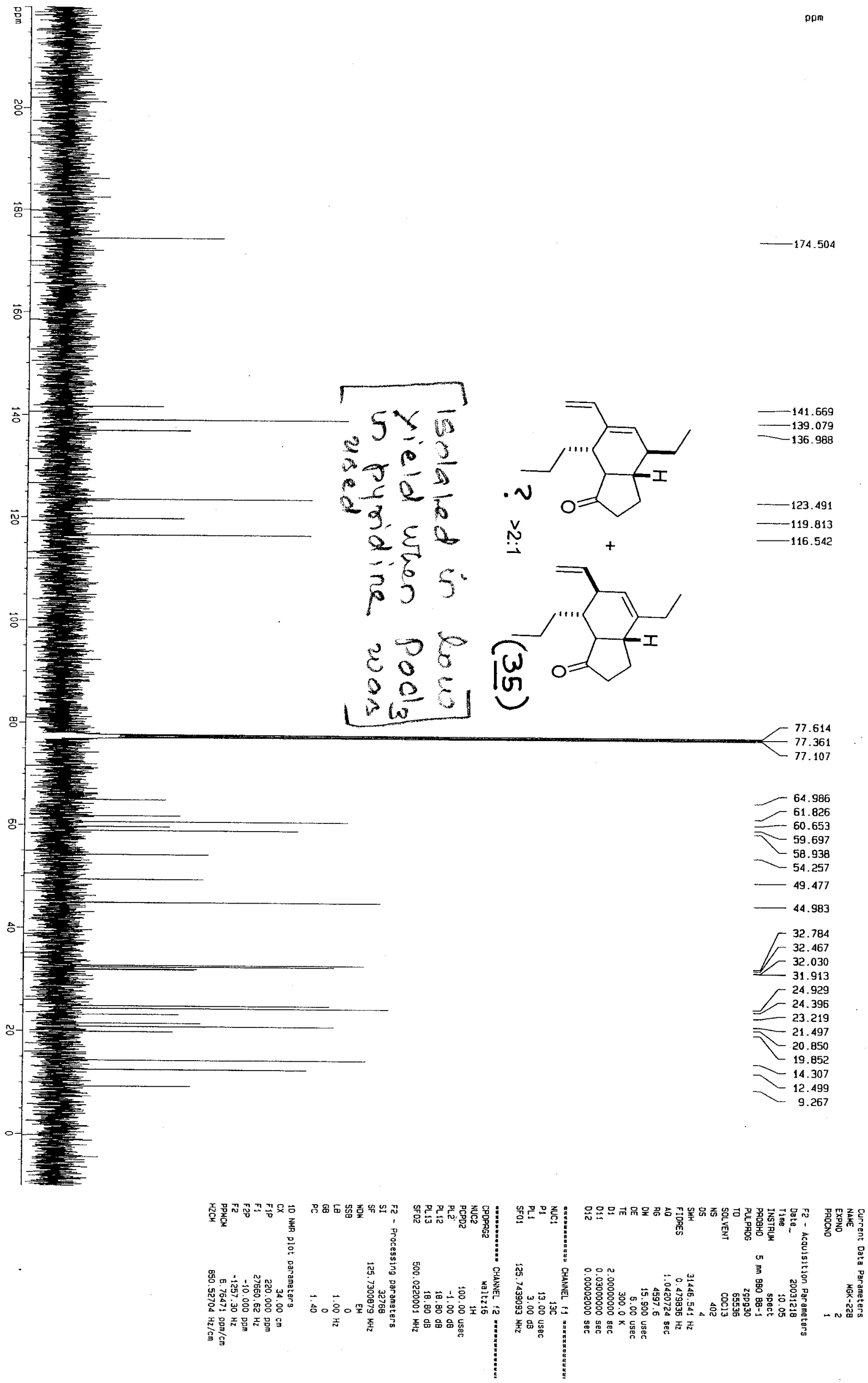

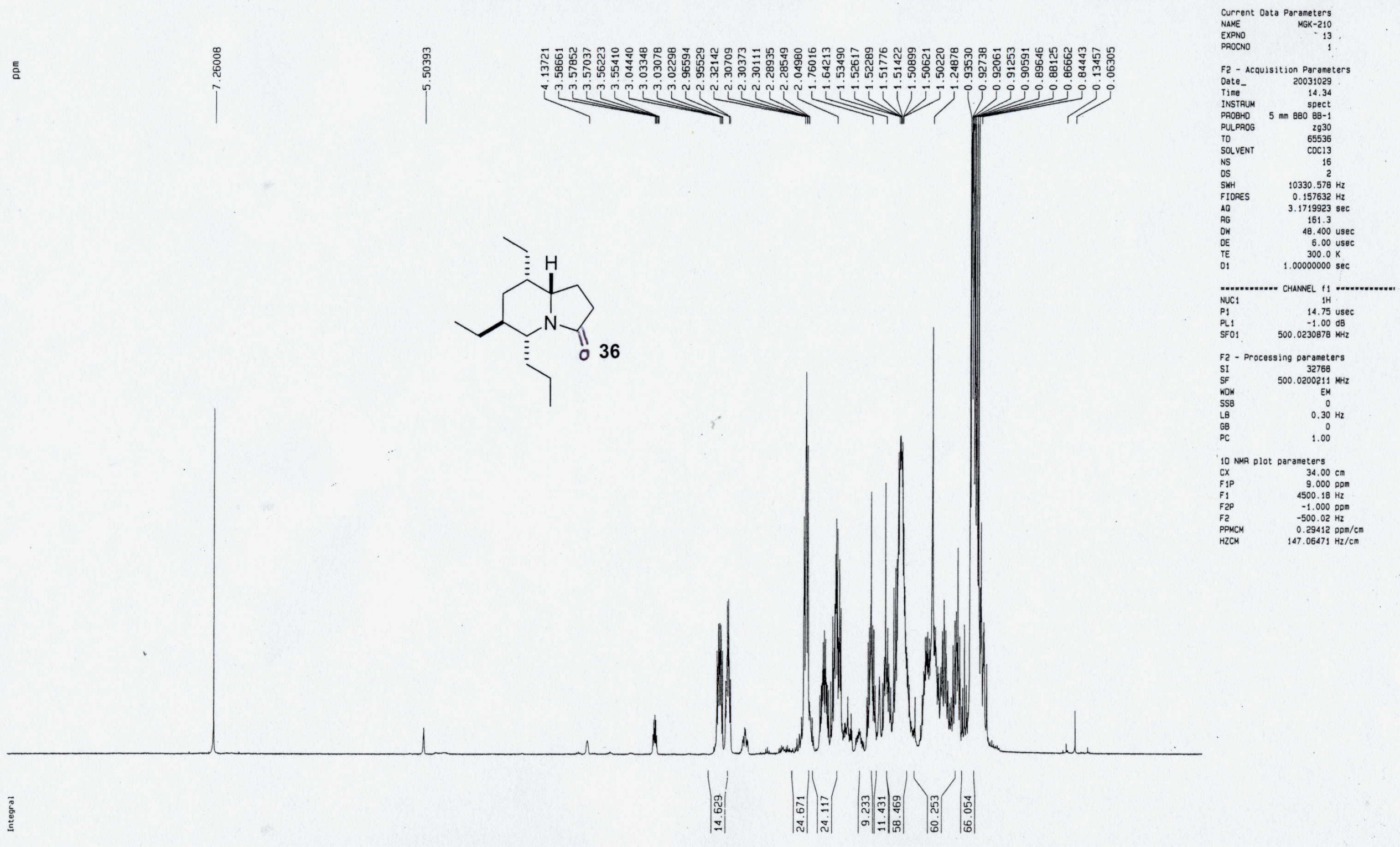

ppm

3

1 


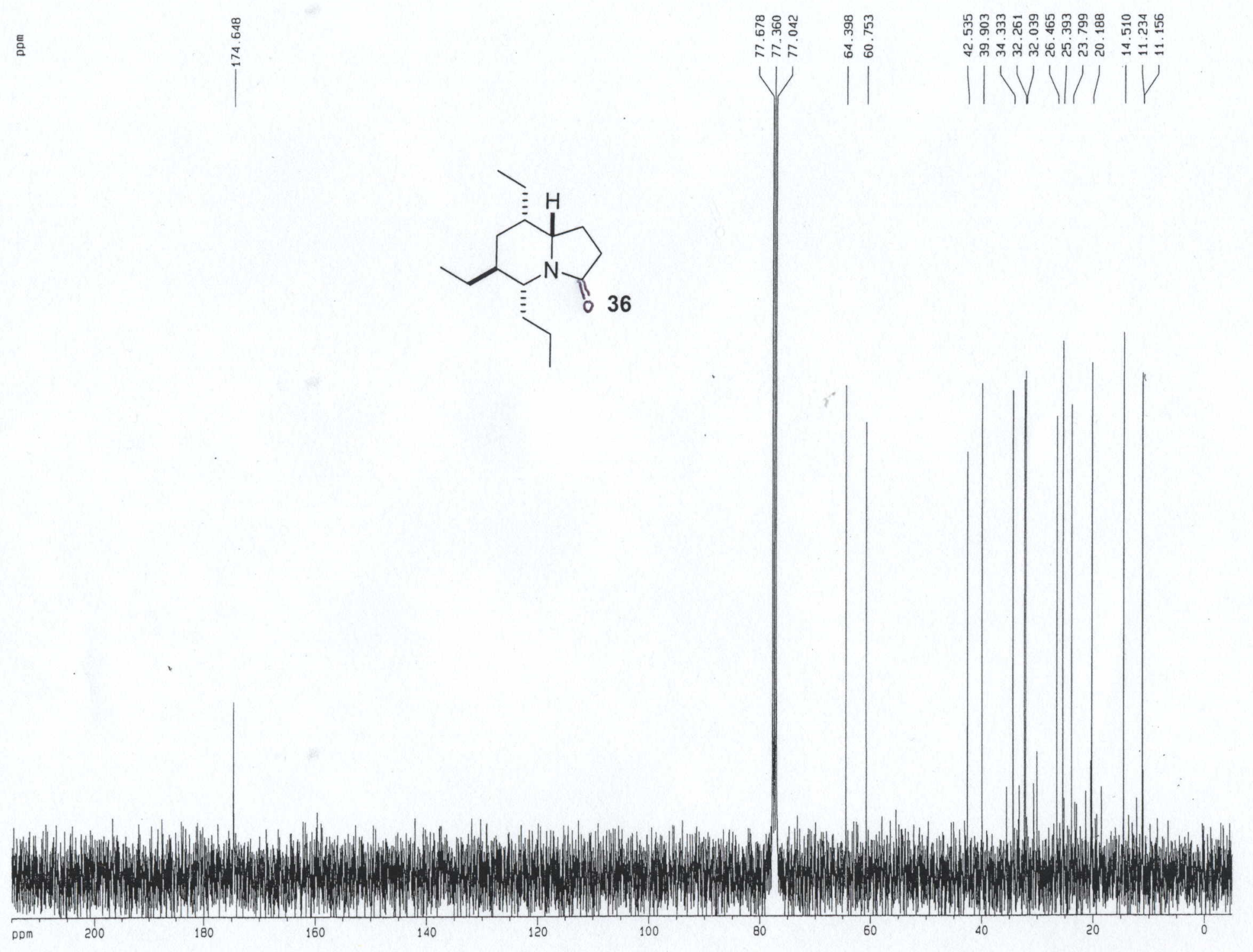

Current Data Parameters

NAME GK-210
10

F2 - Acquisition Parameters

Date_ 20031029

INSTRUM 16,28

PROBHD $5 \mathrm{~mm} \mathrm{B8O} \mathrm{BB-}$

$\begin{array}{lr}\text { PULPROG } & \text { 2gpg } 30 \\ \text { TD } & 65536\end{array}$

$\begin{array}{ll}\text { TO } & 65536 \\ \text { SOLVENT } & \text { CDC13 }\end{array}$

$\begin{array}{lr}\text { NS } & 229 \\ \text { DS } & 4\end{array}$

SWH $25125.629 \mathrm{~Hz}$

AQ $\quad 1.3042154 \mathrm{sec}$

RG. 16384

$\begin{array}{lr}\text { DW } & 19.900 \text { usec } \\ \text { DE } & 6.00 \text { usec }\end{array}$

TE $300.0 \mathrm{~K}$

$\begin{array}{ll}01 & 2.00000000 \mathrm{sec} \\ 011 & 0.03000000 \mathrm{sec} \\ 012 & 0.00002000 \mathrm{sec}\end{array}$

$==z=z=3=n=$ CHANNEL $f 1$

$\begin{array}{ll}\text { NUC1 } & 13 C \\ P_{1} & 5.90 \text { use }\end{array}$

$\begin{array}{lr}\text { P1 } & 5.90 \text { use } \\ \text { PL1 } & -6.00 \mathrm{OB}\end{array}$

SF01 $\quad 100.6237959$ MHz

$==m=== \pm=$ CHANNEL
CPDPRG
waltz

$\begin{array}{lr}\text { NUC2 } & 1 \mathrm{H} \\ \text { PCPD2 } & 80.00 \text { us }\end{array}$

PL2 $\quad-6.00 \mathrm{~dB}$

$\begin{array}{lr}\text { PL12 } & 15.80 \mathrm{~dB} \\ \text { PL13 } & 15.80 \mathrm{~dB}\end{array}$

F2 - processing parameters

SI 32768

SF $\quad 100.6127332 \mathrm{MHz}$

$\begin{array}{lc}\text { WOW } & E M \\ 5 S B & 0 \\ 5 B & 1000\end{array}$

$\begin{array}{ll}L B & 1.00 \mathrm{~Hz} \\ \text { GB } & 1.00 \\ \text { PC } & 1.40\end{array}$

10 NMR plot parameters

CX $\quad 30.00 \mathrm{~cm}$

F1P $\quad 215,000 \mathrm{ppm}$

$\begin{array}{lr}\text { F1 } & 21631.74 \mathrm{~Hz} \\ \text { F2P } & -5.000 \mathrm{DOm}\end{array}$

$\begin{array}{ll}\text { F2 } & -503.06 \mathrm{~Hz} \\ \text { PPMCM } & 7.33333\end{array}$

$\begin{array}{ll}\text { PPMCM } & 737.33333 \mathrm{pDm} / \mathrm{Cm} \\ \mathrm{HZCM} & 732672 \mathrm{~Hz} / \mathrm{cm}\end{array}$ 


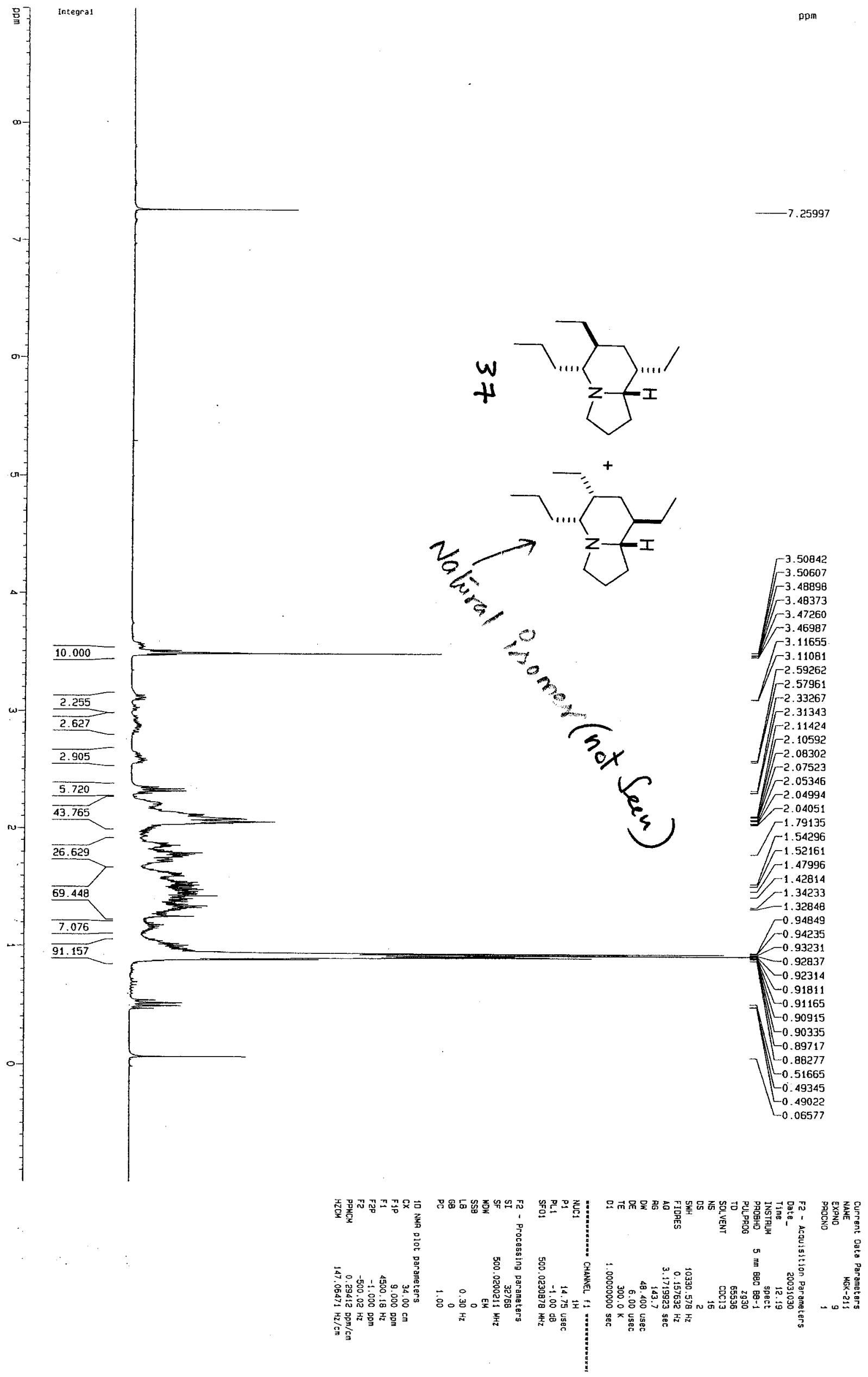




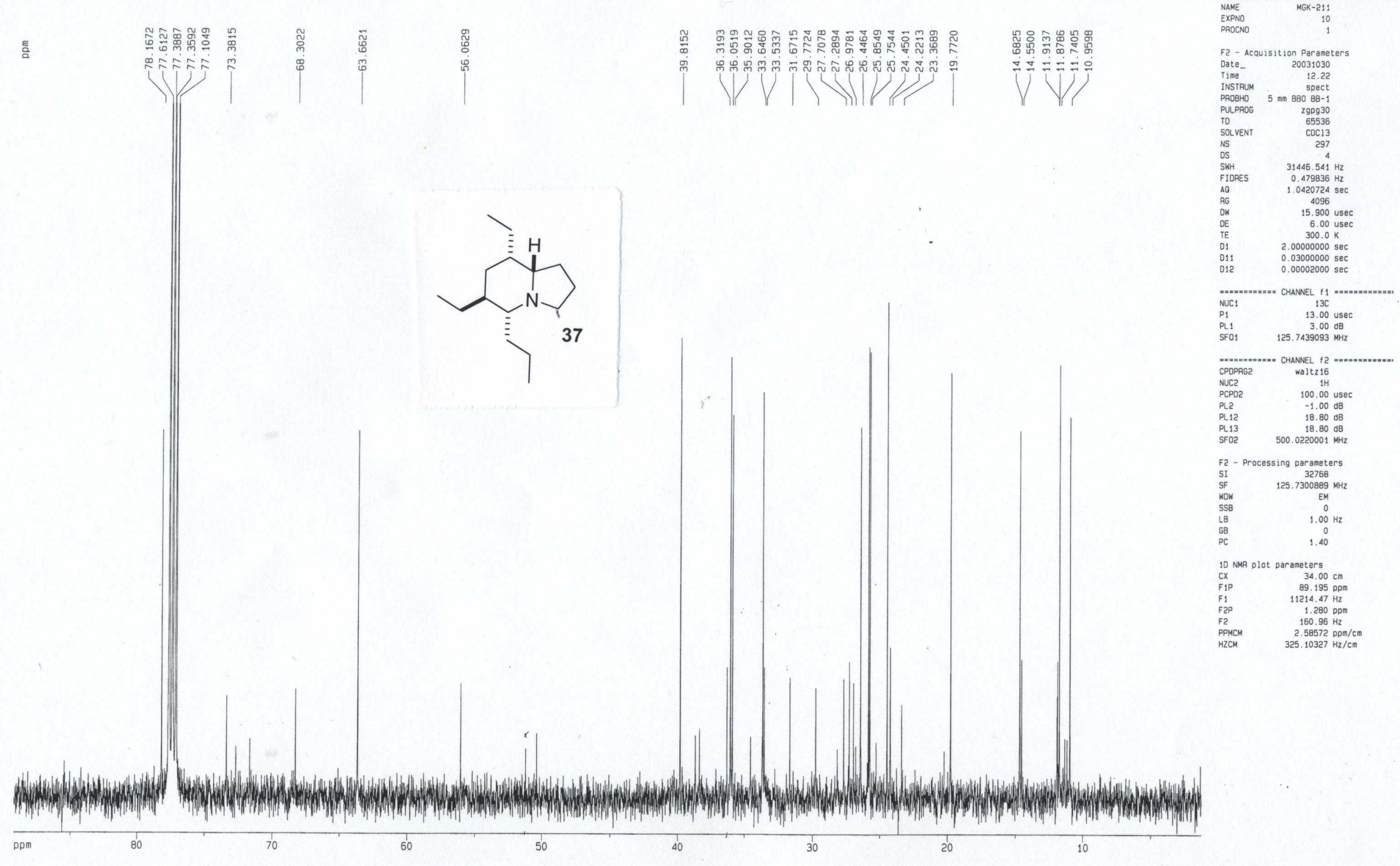




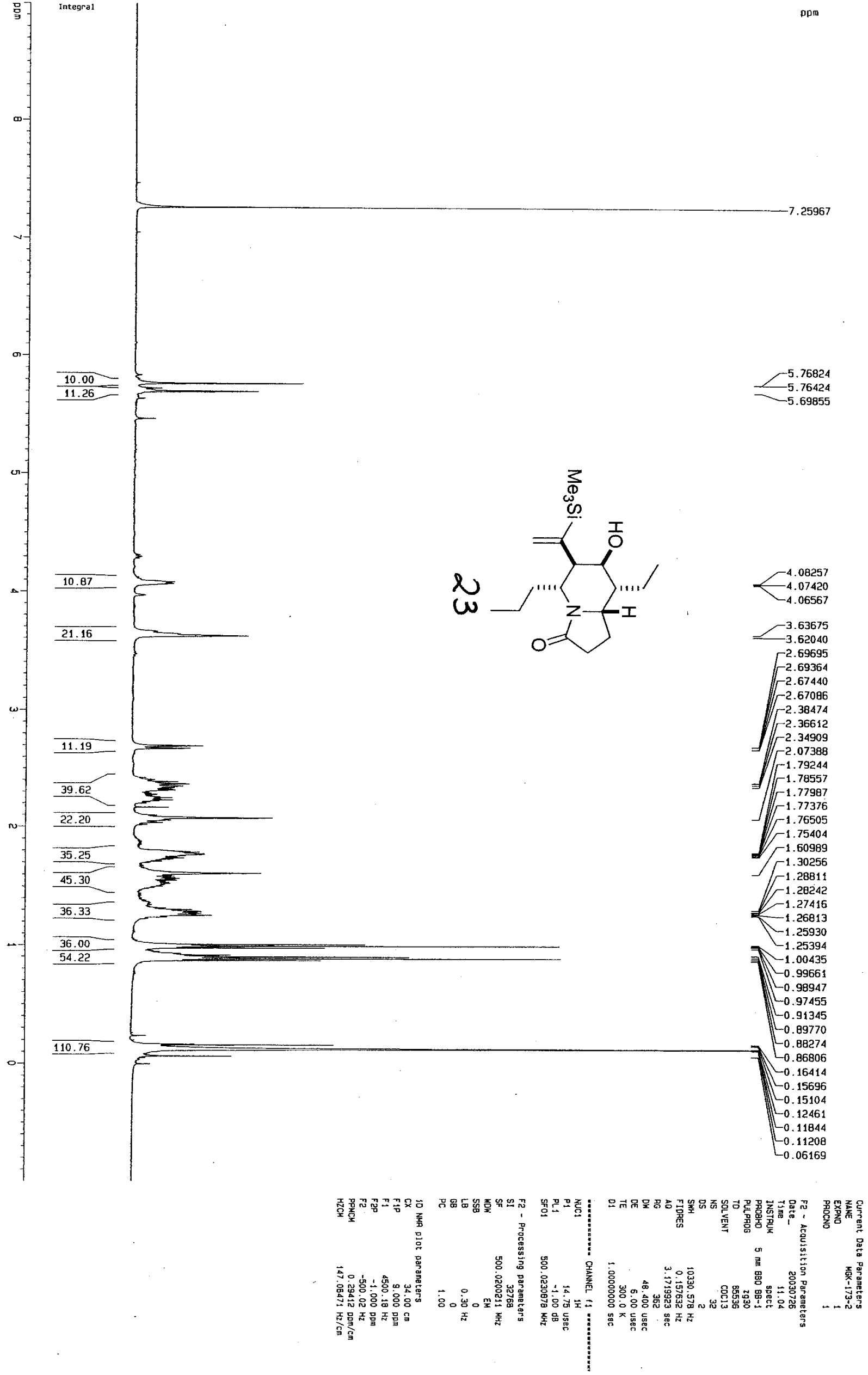




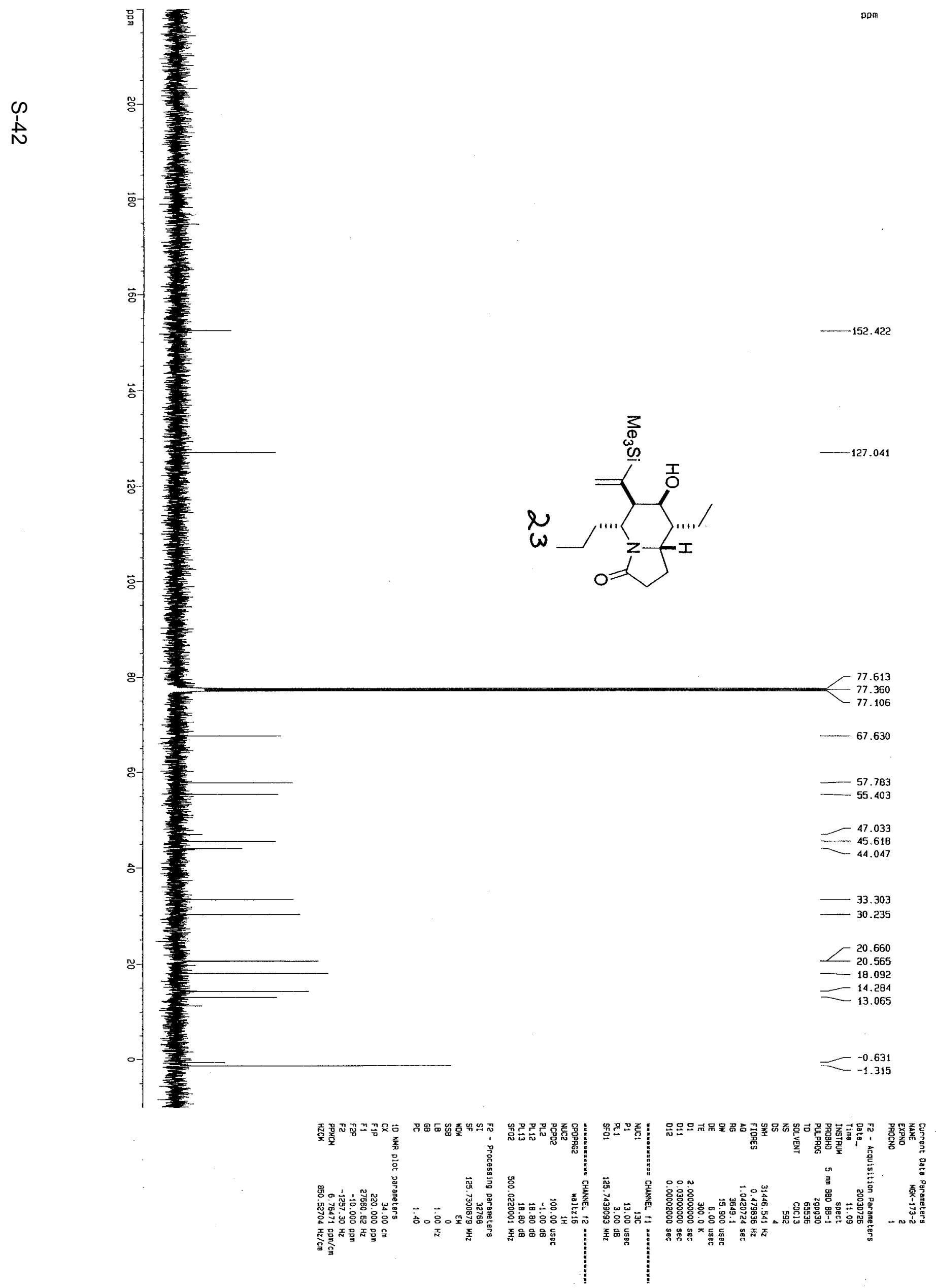




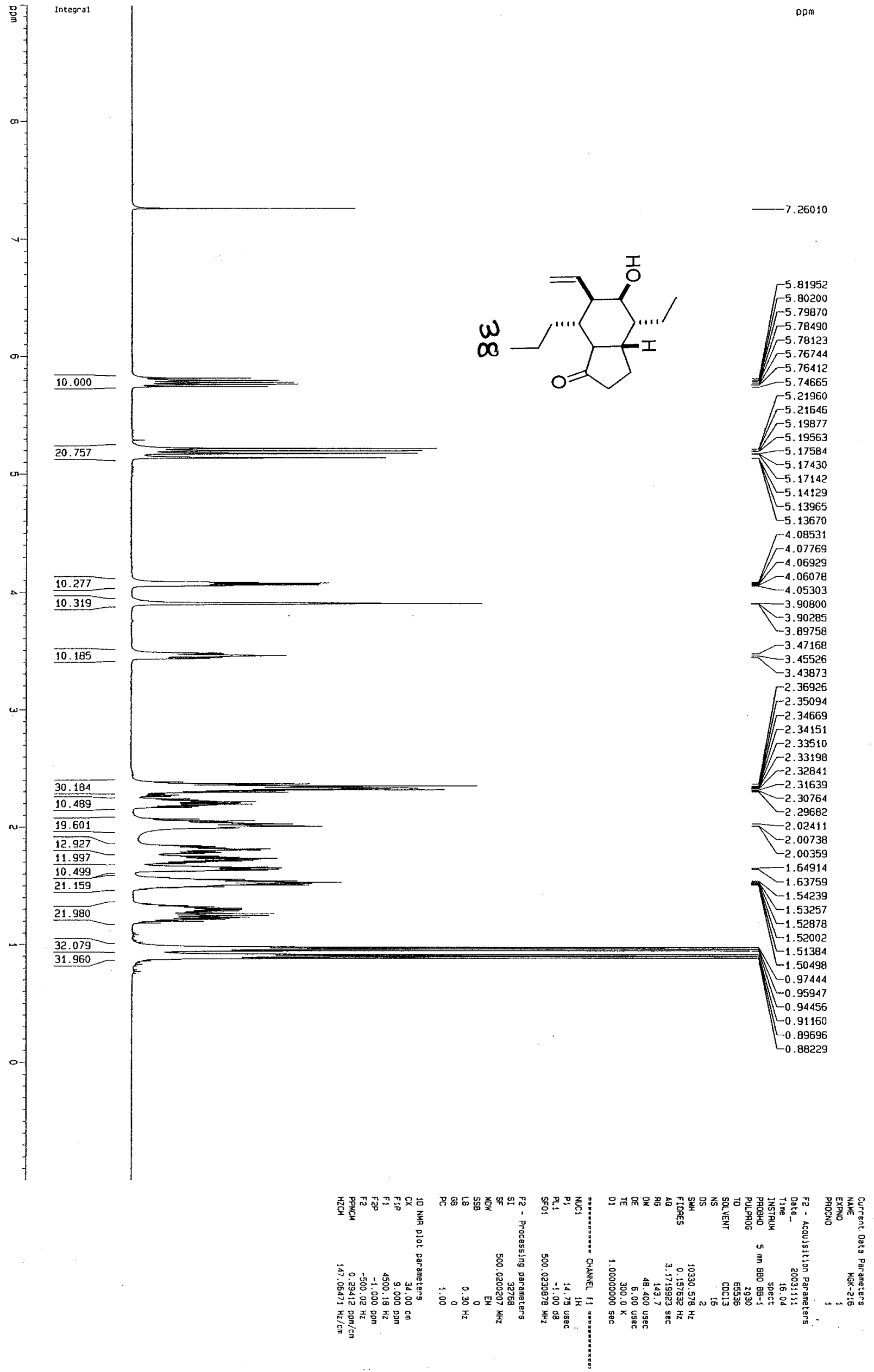




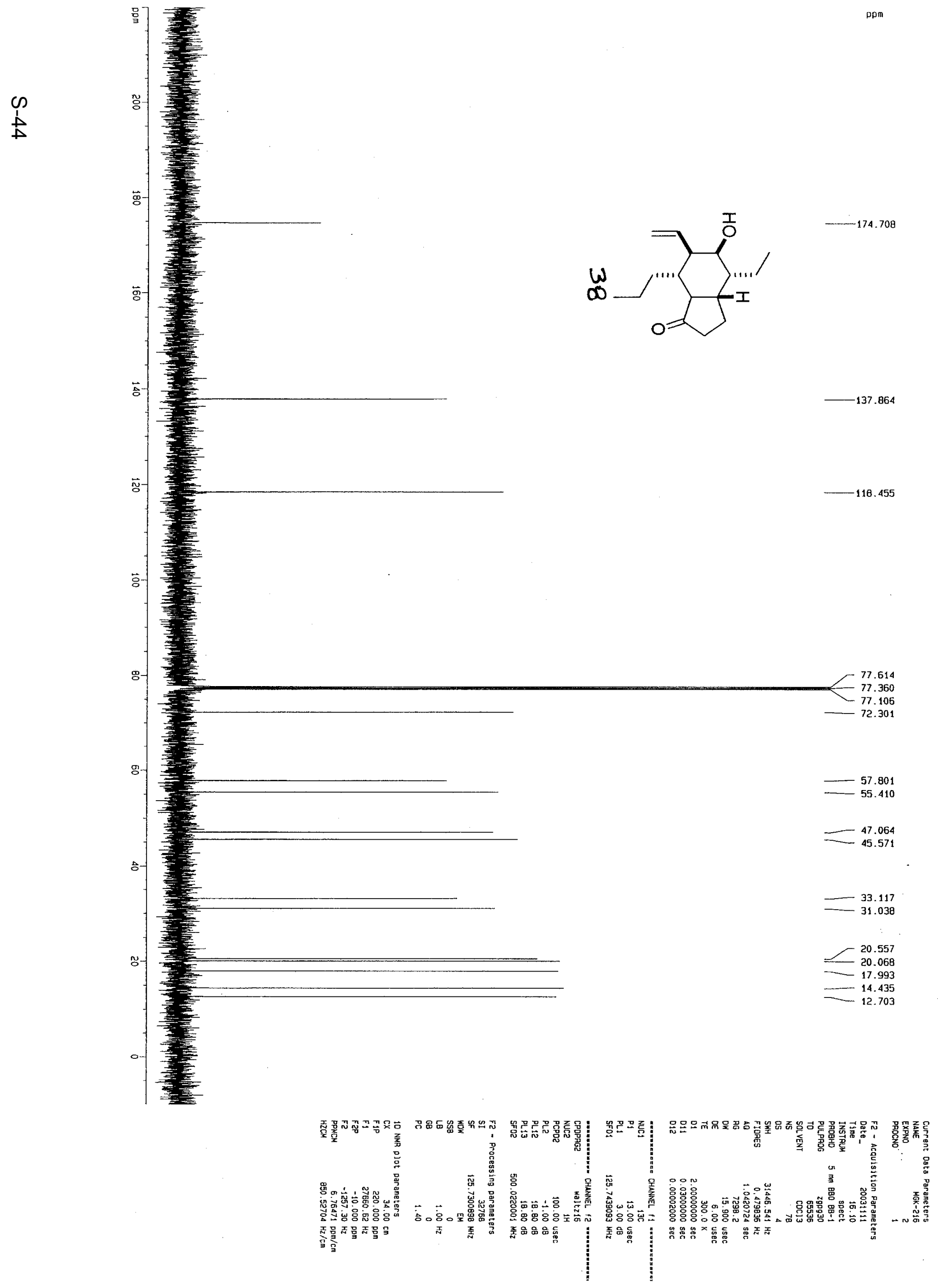




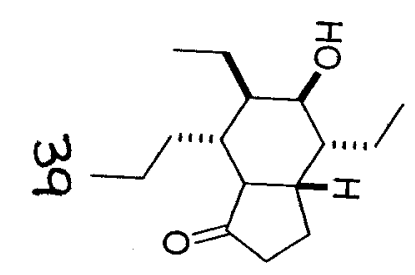

$\overline{19.068-}$

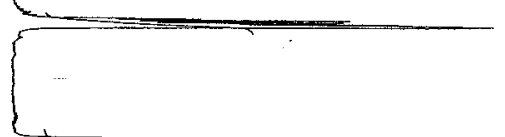

$\left[\begin{array}{r}4.0530 \\ 4.0434\end{array}\right.$ 4.0434 - 4.0330 $-4.0224$ 4.0121 4.0075
$-\quad 3.2862$
-3.2704 3.0075
-3.2862
-3.2704 3.2704
-3.2631 3.2631
-3.2474 3.2383
-3.2384 3.2383
-2.2808 2. 2808 - 2.2689 - 2.2583 r 2.2443 r 1.6305 $\int F_{1} 1.5004$

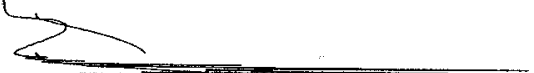

1.4794
-1.5004
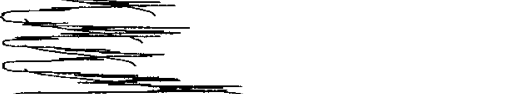

$\frac{10.148}{20.545}$ 40.130 33.737 90.000

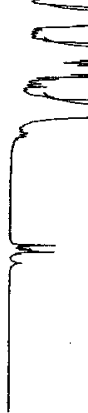

-0.9140
-0.9059

$-0.9031$

$\left[\begin{array}{l}0.8987 \\ 0.8832\end{array}\right.$

$-0.8832$

$-0.8803$

$\left[\begin{array}{ll}0.8647 \\ -0.0434\end{array}\right.$

L 0.0410

$-0.0002$

$-0.0026$

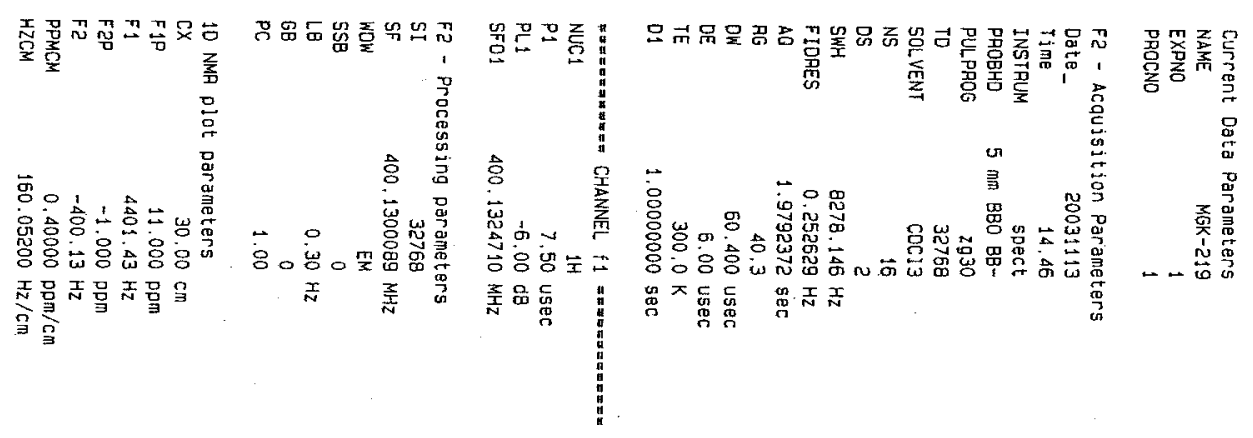


c

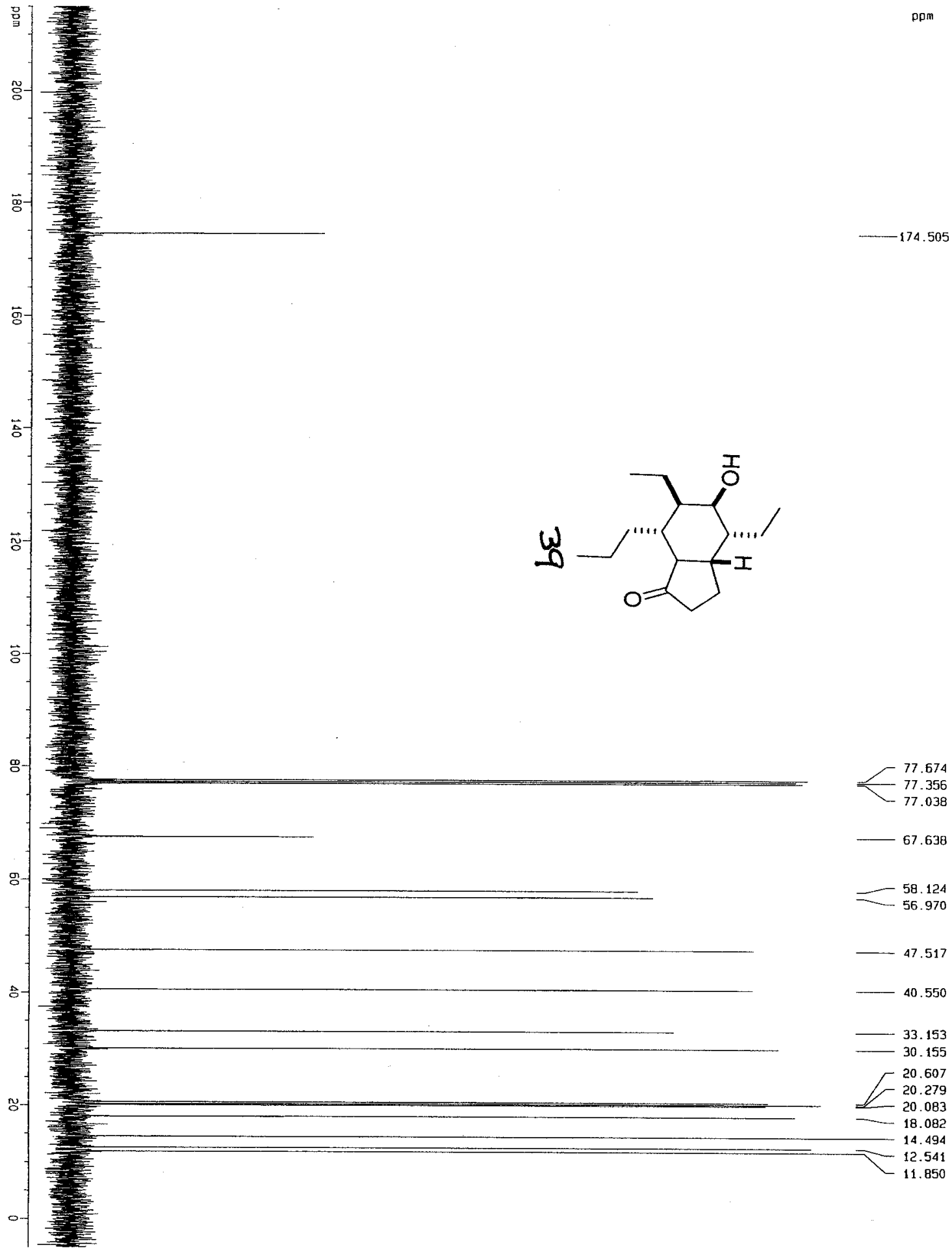

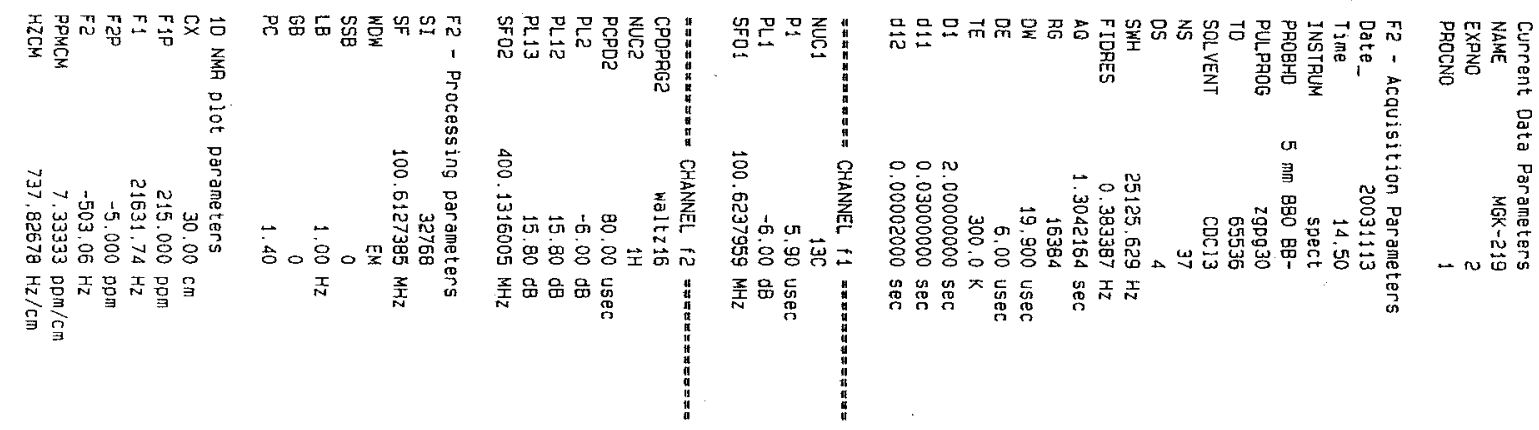




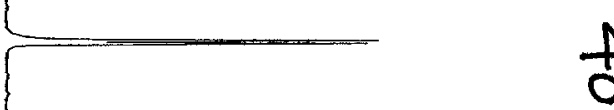

$\frac{1}{0}$
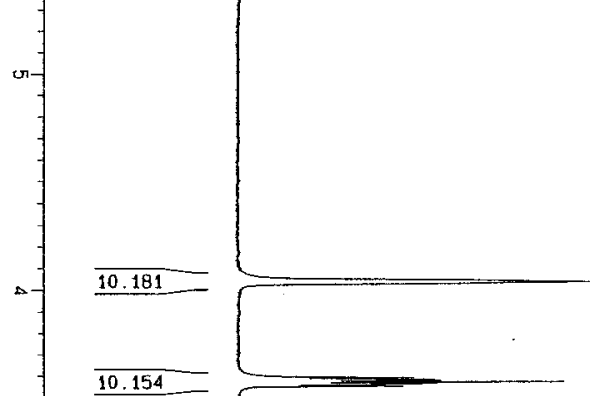

10.154

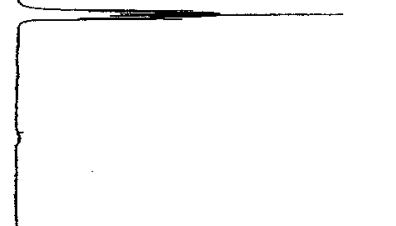
10.922

21.906

11.924

22.266

30.958

36.666

$\frac{12.652}{33.055}$
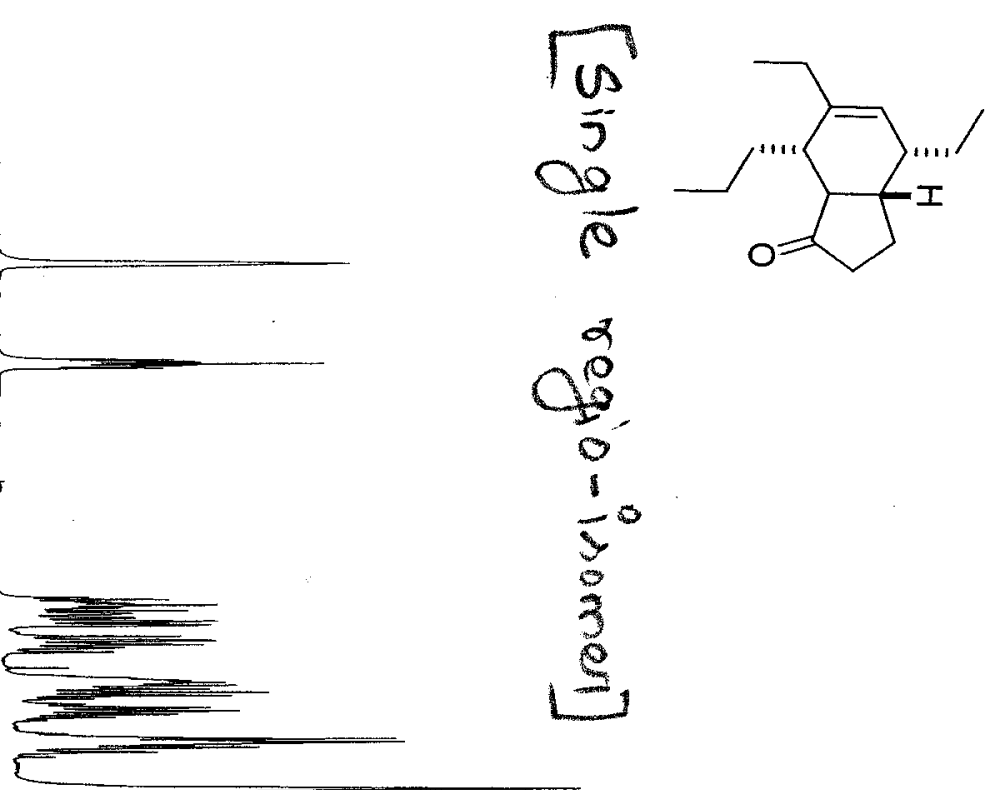

$\alpha$
0
10
0

?

$\pm$

5

3

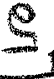
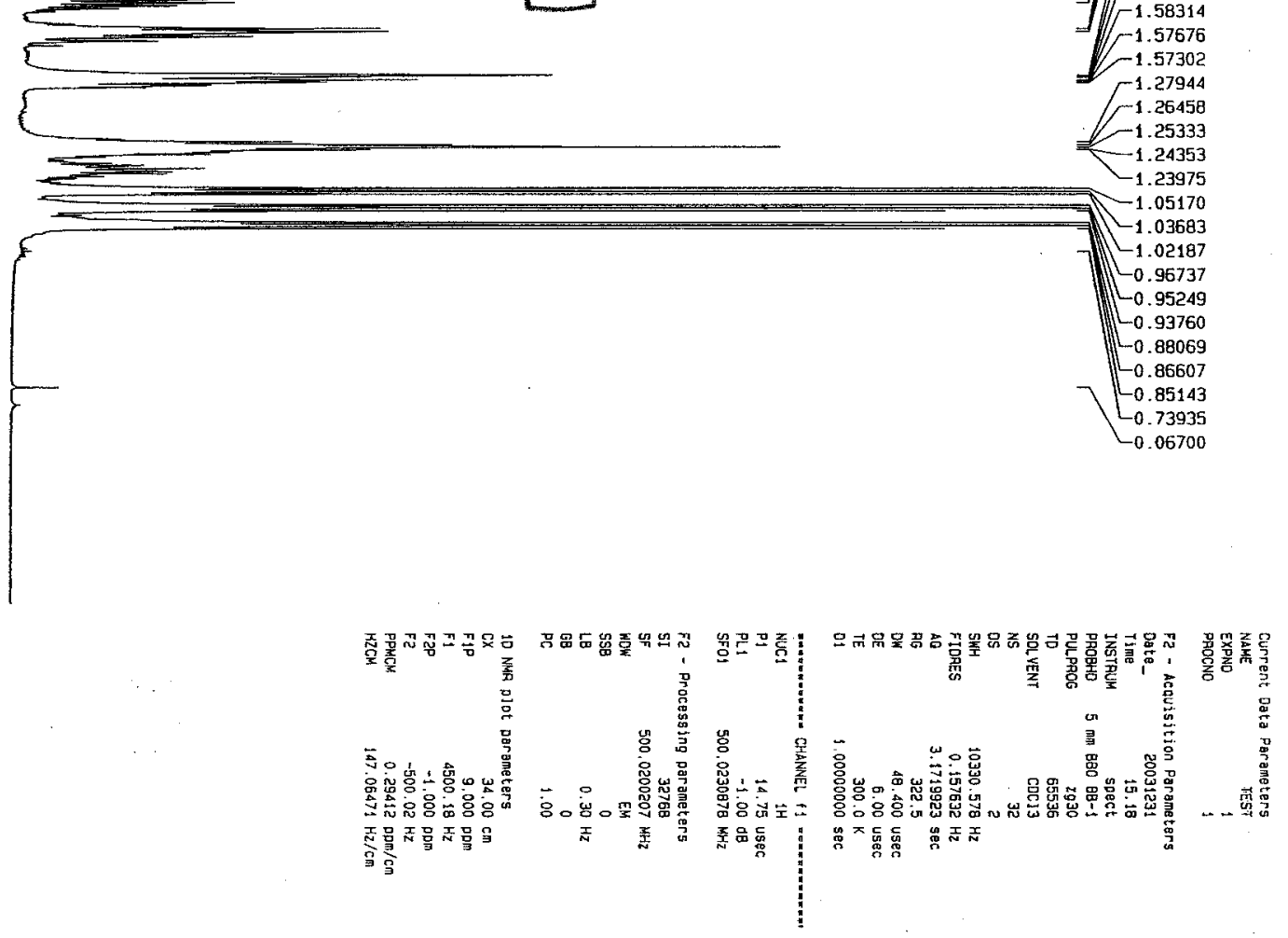
$\infty$
1
$\infty$

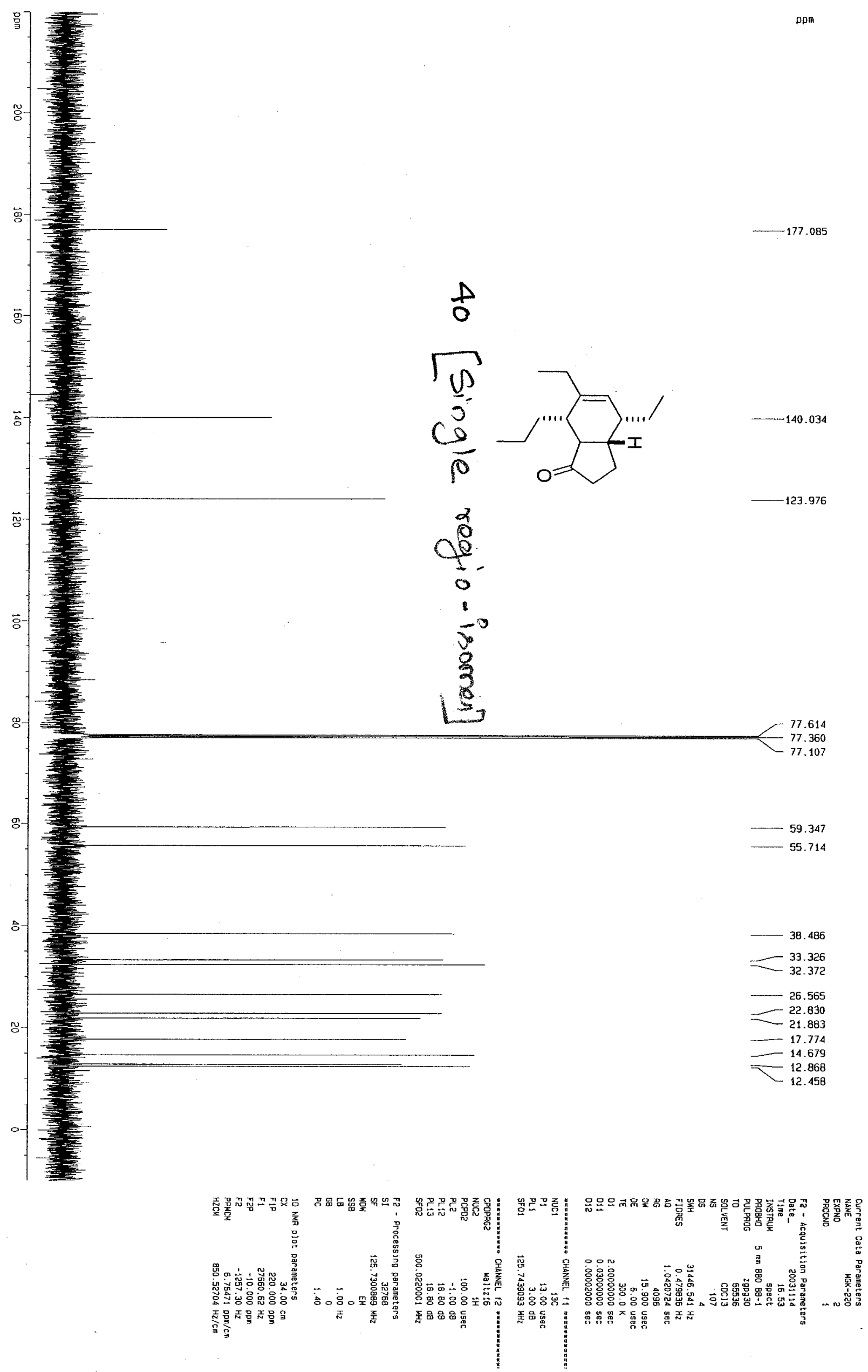


$c$
1
0
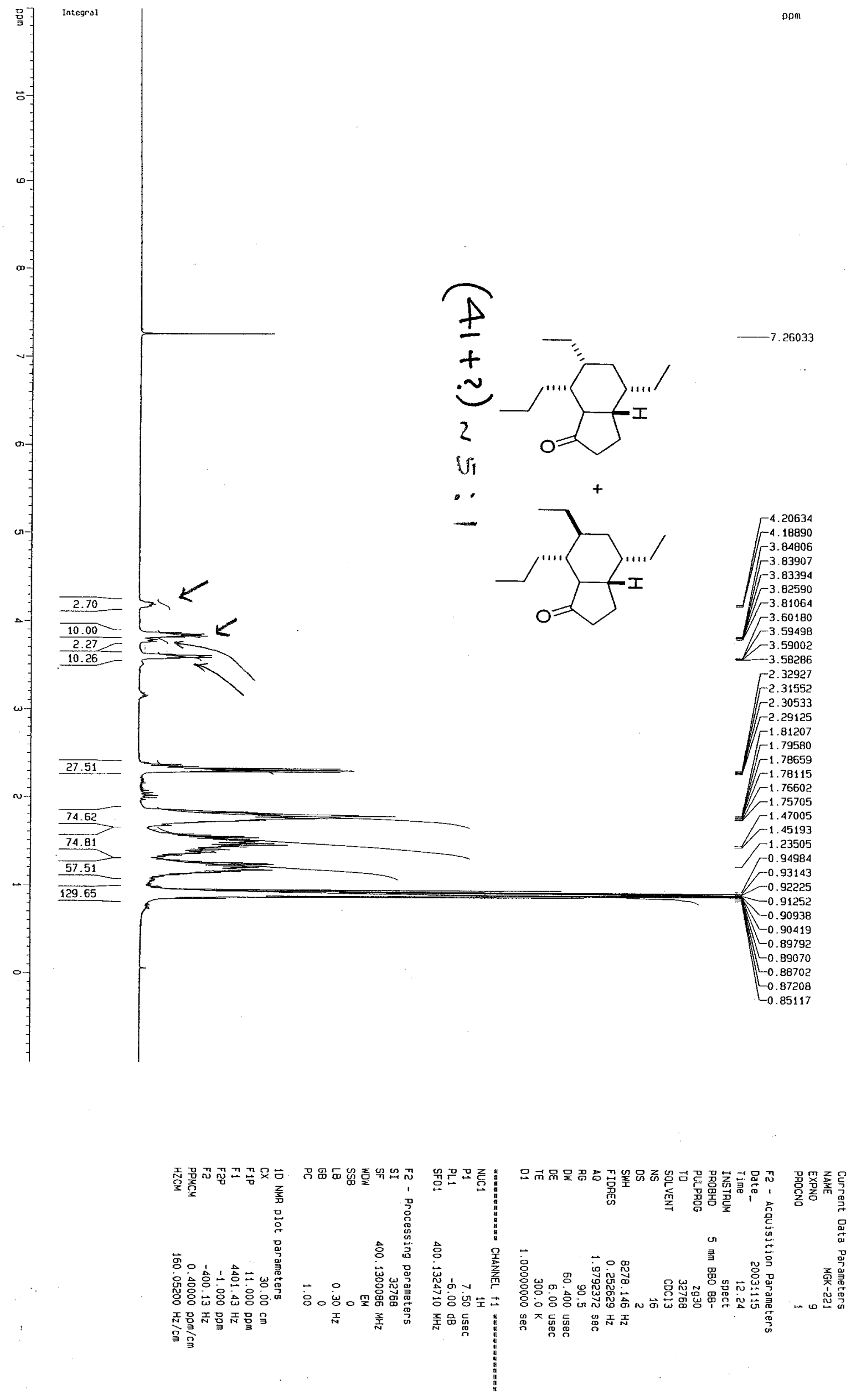
c)

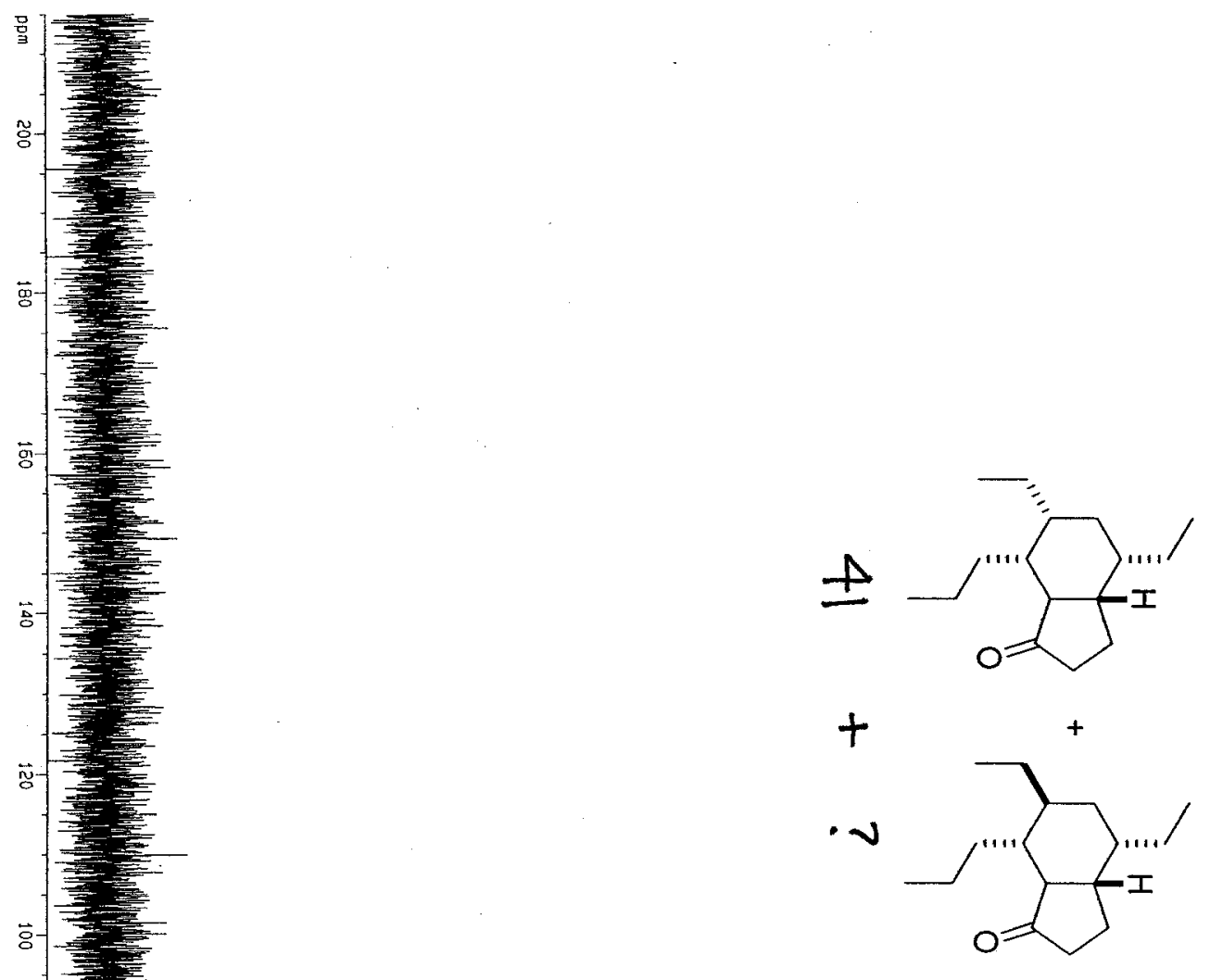

g-

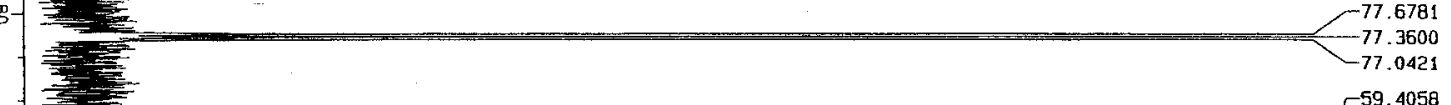

$-59.4058$

\begin{tabular}{r}
56.5536 \\
55.2384 \\
-50.9283 \\
\hline \\
-30.5696
\end{tabular}

$-40.1399$

1) 38.5686

-36.6868

(Ir. 3599

$\mid\left(\Gamma_{-31.2297}^{32.1736}\right.$

Jill -28.6525

28.1138

घ) 26.3221

I) 24.7625

$-23.0743$

ก-

22. 1749

$-20.4555$

$-19.4398$

$-17.1921$

$-14.7601$

$-14.4025$

12. 6876

$-12.2523$

$-12.0206$

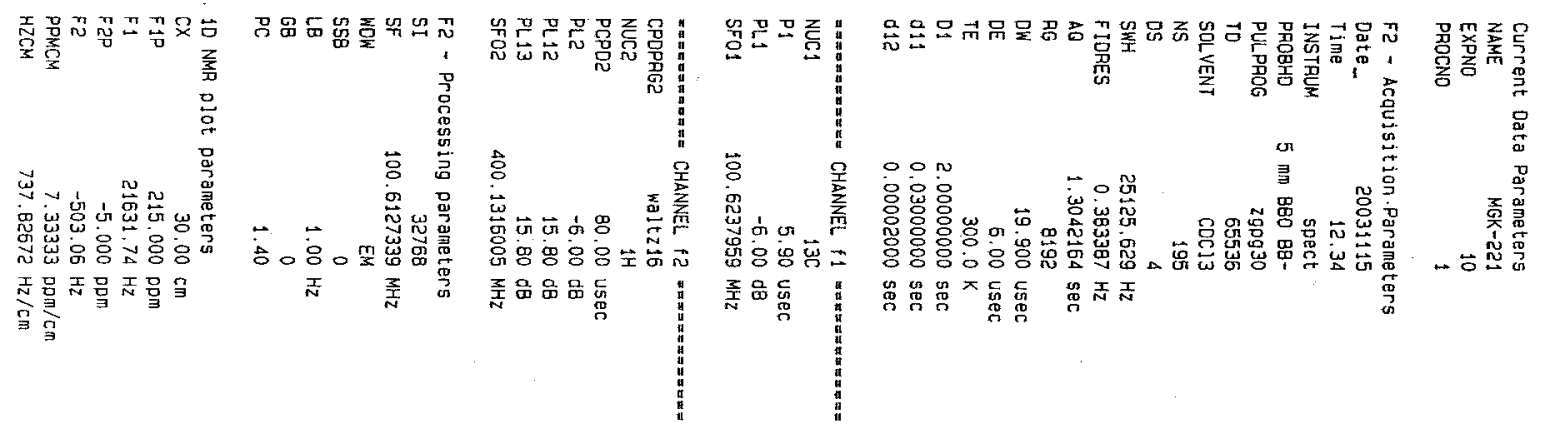




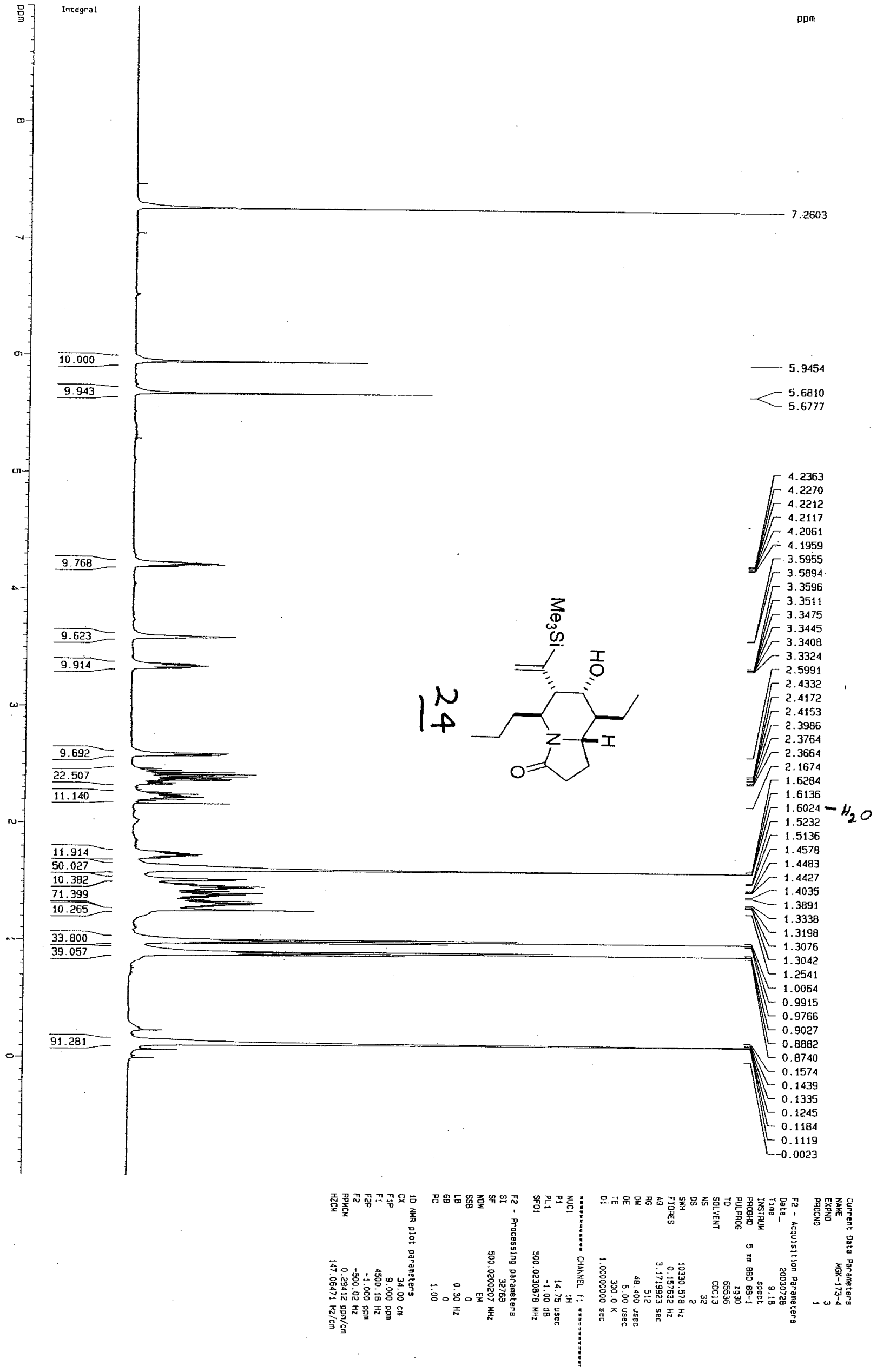


co

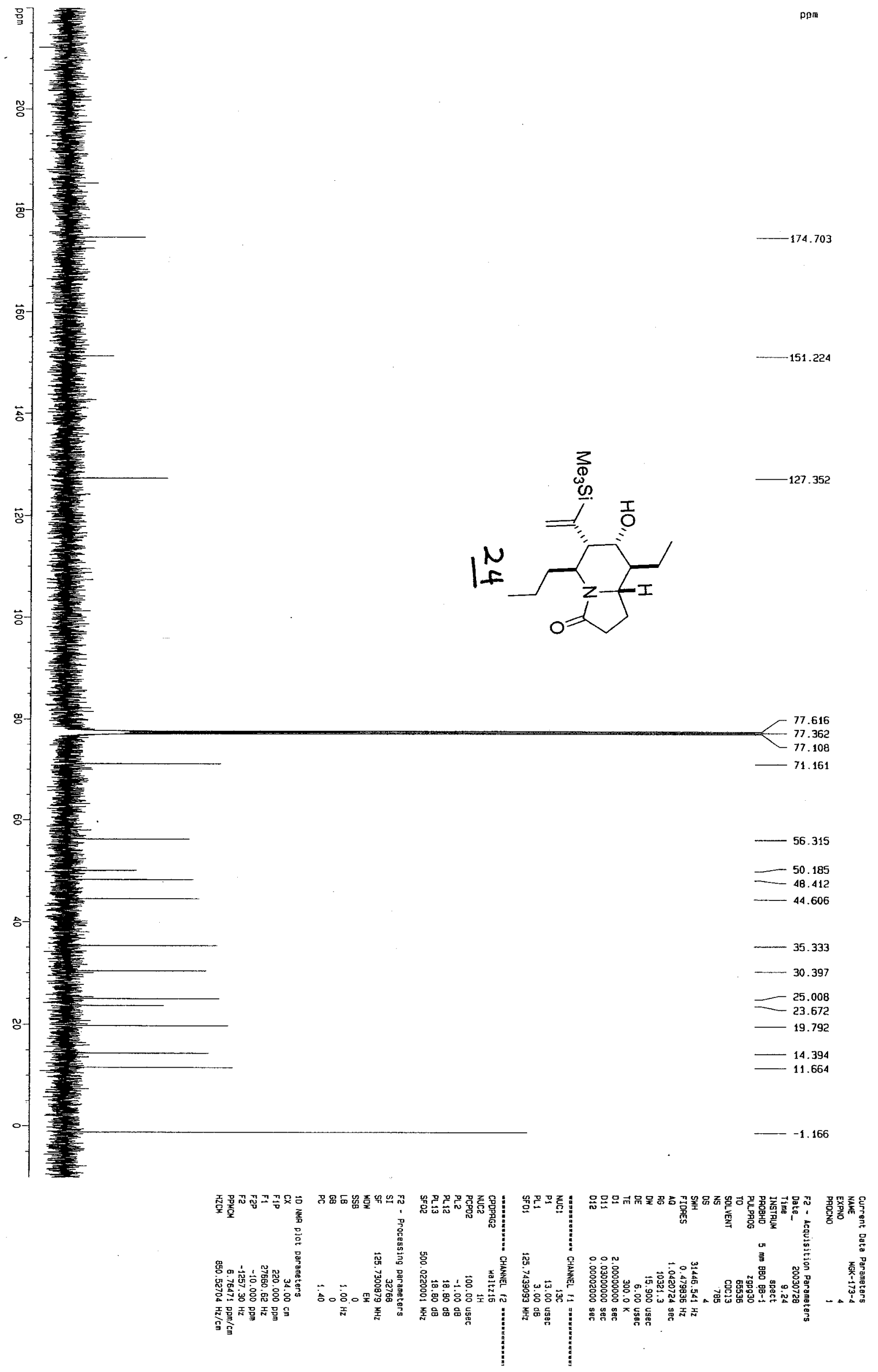




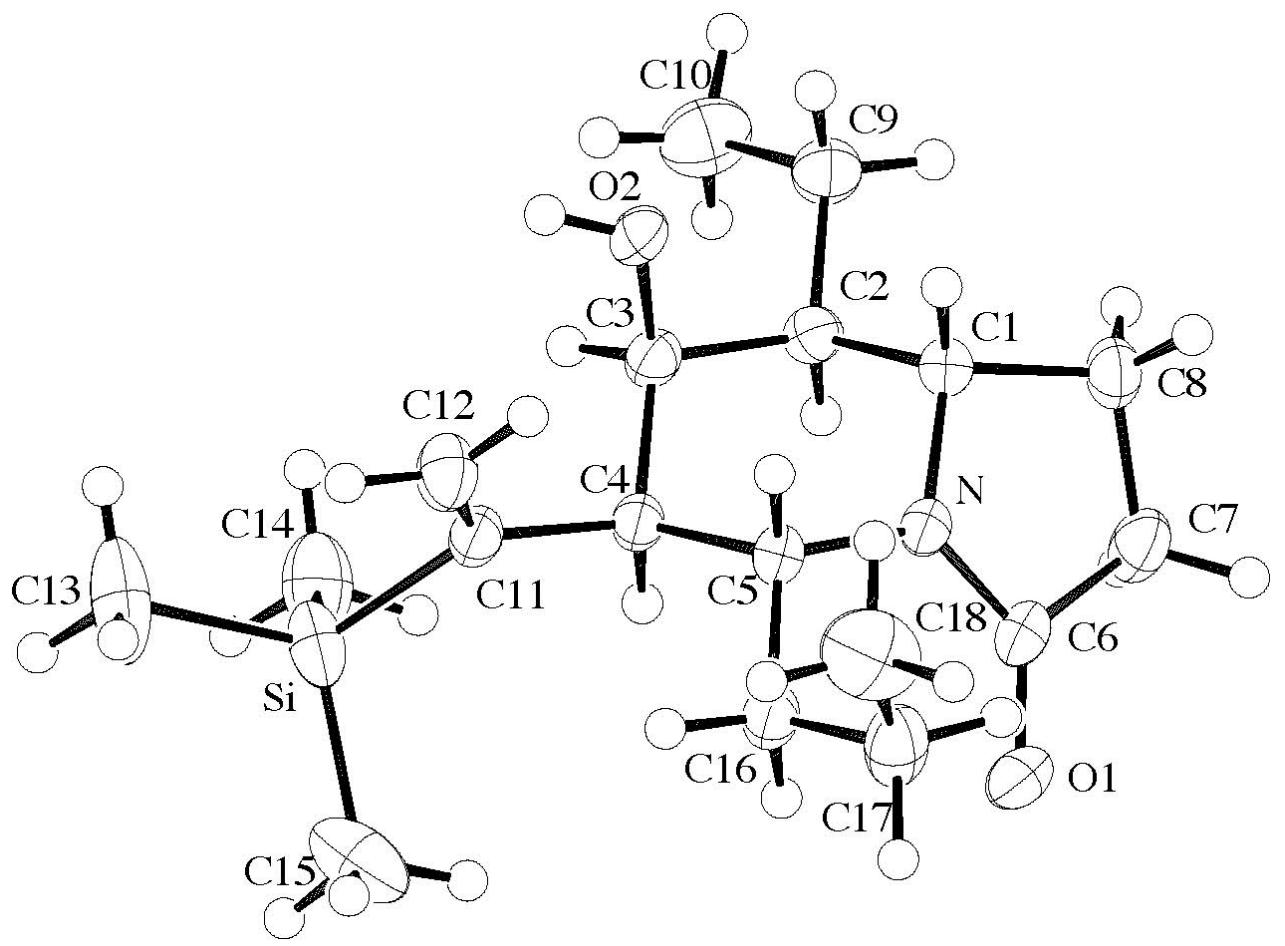

ORTEP of $\mathbf{2 1}$
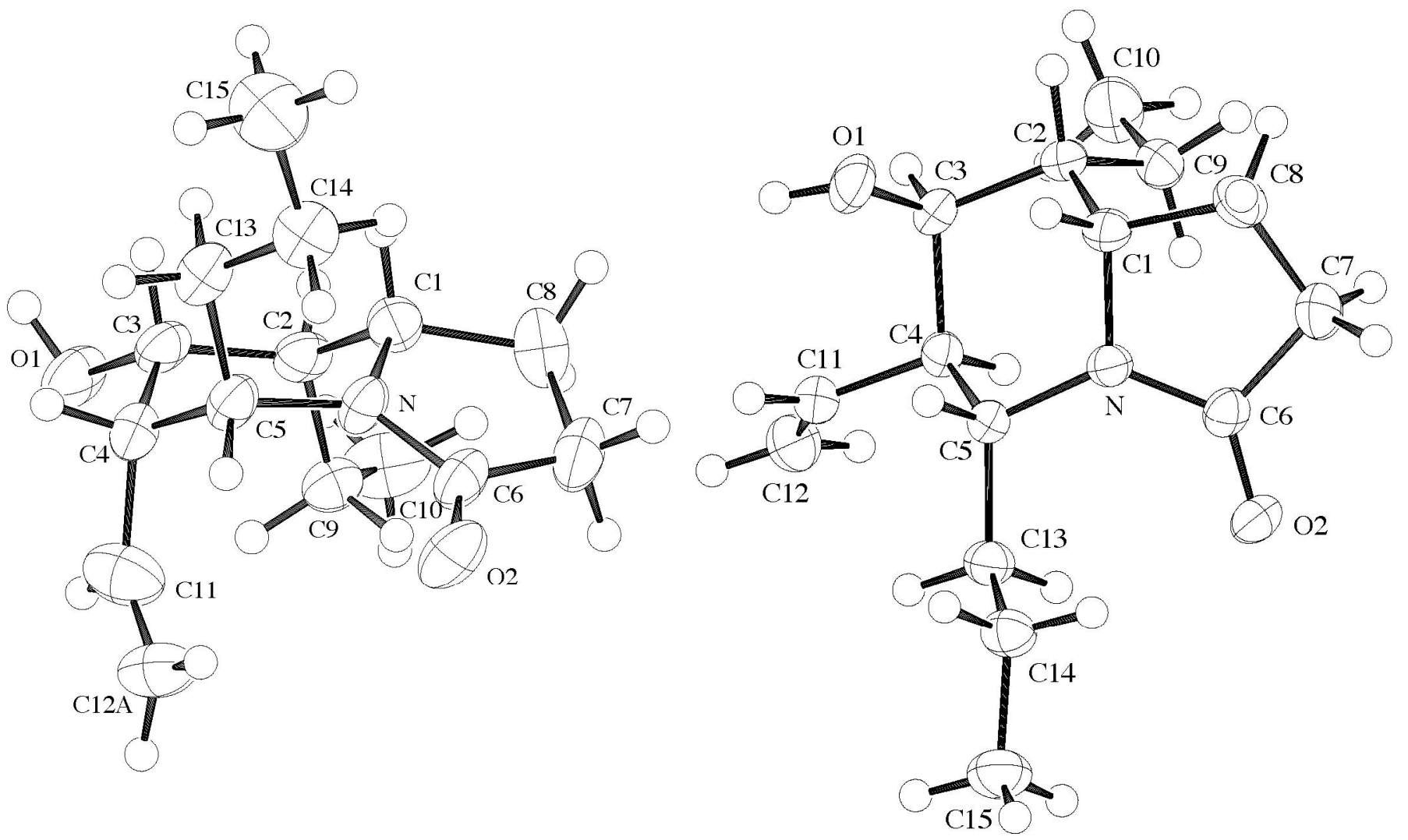

ORTEP of $\mathbf{2 8}$

ORTEP of $\mathbf{3 8}$ 